\title{
Search for Heavy Higgs Bosons Decaying into Two Tau Leptons with the ATLAS Detector Using $p p$ Collisions at $\sqrt{s}=13 \mathrm{TeV}$
}

\author{
G. Aad et al. \\ (ATLAS Collaboration)
}

\author{
(Received 28 February 2020; accepted 26 June 2020; published 27 July 2020)
}

\begin{abstract}
A search for heavy neutral Higgs bosons is performed using the LHC Run 2 data, corresponding to an integrated luminosity of $139 \mathrm{fb}^{-1}$ of proton-proton collisions at $\sqrt{s}=13 \mathrm{TeV}$ recorded with the ATLAS detector. The search for heavy resonances is performed over the mass range $0.2-2.5 \mathrm{TeV}$ for the $\tau^{+} \tau^{-}$decay with at least one $\tau$-lepton decaying into final states with hadrons. The data are in good agreement with the background prediction of the standard model. In the $M_{h}^{125}$ scenario of the minimal supersymmetric standard model, values of $\tan \beta>8$ and $\tan \beta>21$ are excluded at the $95 \%$ confidence level for neutral Higgs boson masses of 1.0 and $1.5 \mathrm{TeV}$, respectively, where $\tan \beta$ is the ratio of the vacuum expectation values of the two Higgs doublets.
\end{abstract}

DOI: 10.1103/PhysRevLett.125.051801

The ATLAS and CMS collaborations discovered in 2012 a new boson with a mass of $125 \mathrm{GeV}$ [1,2]. Current measurements $[3,4]$ indicate that the new particle is compatible with the Higgs boson predicted by the standard model (SM) [5-7]. This discovery opens the way for studies of the structure of the Higgs sector. Many theoretical models beyond the SM, such as two-Higgs-doublet models (2HDMs) [8], extend the Higgs sector to include a second Higgs doublet which implies the existence of new heavy pseudoscalar $(A)$ and scalar $(H)$ states, while the observed scalar particle would correspond to the lightest Higgs boson $(h)$. The decay probability of these scalar states into $\tau^{+} \tau^{-}$pairs can be enhanced relative to other decay modes in 2HDMs of type II, such as the minimal supersymmetric SM (MSSM) [9,10], the minimal extension of the SM that realizes supersymmetry [11-16].

At tree level, the properties of the MSSM Higgs sector depend only on two non-SM parameters, which can be chosen to be the mass of the pseudoscalar Higgs boson, $m_{A}$, and the ratio of the vacuum expectation values of the two Higgs doublets, $\tan \beta$. Beyond tree level, the Higgs sector is affected by additional parameters, the choice of which defines various MSSM benchmark scenarios. In the $M_{h}^{125}$ scenario [17], the parameters are such that the mass of the lightest $C P$-even Higgs boson, $m_{h}$, is close to the measured mass of the Higgs boson discovered at the LHC [18] and the masses of all superparticles are heavy enough to only

\footnotetext{
*Full author list given at the end of the article.

Published by the American Physical Society under the terms of the Creative Commons Attribution 4.0 International license. Further distribution of this work must maintain attribution to the author(s) and the published article's title, journal citation, and DOI. Funded by SCOAP ${ }^{3}$.
}

mildly affect the production and decays of the MSSM Higgs bosons. The couplings of the MSSM heavy Higgs bosons to down-type fermions are enhanced with respect to the SM for large $\tan \beta$ values, resulting in increased branching fractions to $\tau$ leptons and $b$ quarks, as well as a higher cross section for Higgs boson production in association with $b$ quarks $(b b H)$. For the mass range considered in this Letter, the mass difference between the $A$ and $H$ bosons is much smaller than the experimental resolution and they are treated as degenerate in mass.

This Letter describes a search for massive scalar and pseudoscalar resonances decaying into a $\tau$-lepton pair (throughout this Letter the inclusion of charge-conjugate decay modes is implied). The search is conducted on a sample of proton-proton collision data with an integrated luminosity of $139 \mathrm{fb}^{-1}$ at a center-of-mass energy of $\sqrt{s}=13 \mathrm{TeV}$, collected with the ATLAS detector [1921] during the Run 2 of the LHC (2015-2018) [22]. The $\tau_{\text {lep }} \tau_{\text {had }}$ and $\tau_{\text {had }} \tau_{\text {had }}$ decay channels are considered, where $\tau_{\text {lep }}$ denotes the decay of the $\tau$ lepton into neutrinos and an electron $\left(\tau_{e}\right)$ or into neutrinos and a muon $\left(\tau_{\mu}\right)$ and $\tau_{\text {had }}$ denotes the decay into a neutrino and hadrons. This search improves on the results obtained by previous searches performed by the ATLAS and CMS collaborations at a center-of-mass energy of $\sqrt{s}=13 \mathrm{TeV}$ [23-25] by about a factor of 4-5 for a scalar boson in the mass range 700 $2500 \mathrm{GeV}$, thanks to improvements of the modeling of the top-quark background and of the backgrounds estimated from data, of the reconstruction of high- $p_{T} \tau$ leptons and the increase of integrated lumnosity.

The ATLAS detector at the LHC covers nearly the entire solid angle around the collision point [26]. It consists of an inner tracking detector surrounded by a thin superconducting solenoid, electromagnetic and hadronic calorimeters, 
TABLE I. Generators used to describe the signal and background processes, parton distribution function (PDF) sets for the hard process, and models used for parton showering, hadronization and the underlying event (UEPS). The orders of the total cross sections used to normalize the events are also given. $V$ represents either $W$ or $Z$ gauge bosons.

\begin{tabular}{lllll}
\hline \hline Process & \multicolumn{1}{c}{ Generator } & \multicolumn{1}{c}{ PDF } & \multicolumn{1}{c}{ UEPS } & \multicolumn{1}{c}{ Cross section order } \\
\hline ggF & POWHEG-BOX v2 [65-69] & CT10 [70] & PYTHIA 8.1 [71] & See text \\
$b b H$ & MG5_aMC @ NLO 2.1.2 [72,73] & CT10 & PYTHIA 8.2 [74] & See text \\
$W+$ jets & SHERPA 2.2.1 [75] & NNPDF 3.0 NNLO [76] & SHERPA 2.2.1 [77] & NNLO [78] \\
$Z+$ jets & POWHEG-BOX v1 [65-67,79] & CT10 & PYTHIA 8.1 & NNLO [78] \\
$V V / V \gamma^{*}$ & SHERPA 2.2 & NNPDF 3.0 NNLO & SHERPA 2.2 & NLO \\
$t \bar{t}$ & POWHEG-BOX v2 [65-67,80] & NNPDF 3.0 NLO & PYTHIA 8.2 & NNLO + NNLL [81-87] \\
Single $t$ & POWHEG-BOX v2 [65-67,88-90] & NNPDF 3.0 NLO & PYTHIA 8.2 & NNLO + NNLL [91,92] \\
\hline \hline
\end{tabular}

and a muon spectrometer incorporating three large superconducting toroidal magnets.

Samples of Monte Carlo (MC) simulated events are used to optimize the event selection, estimate the signal efficiencies, and model some of the background processes. The generators and parton showers used to simulate the different MC processes are summarized in Table I. The production cross sections and branching fractions for the various MSSM scenarios are calculated using procedures described in Refs. [27,28]. The cross sections for gluongluon fusion ( $\mathrm{ggF}$ ) production calculated with SUSHI $[29,30]$ include next-to-leading-order (NLO) supersymmetric-QCD corrections [31-36], next-to-next-to-leadingorder (NNLO) QCD corrections for the top quark [37-41], and light-quark electroweak effects $[42,43]$. The $b b H$ cross sections are calculated in the five-flavor [44] and fourflavor schemes $[45,46]$, and the predictions are combined as described in Refs. [47-50]. The other production modes contribute negligibly in the $M_{h}^{125}$ scenario and are not considered. The masses and mixing (and effective Yukawa couplings) of the Higgs bosons are computed with FEYNHIGGS [51-58]. Branching fractions of Higgs bosons are computed using a combination of results calculated by FEYNHIGGS, HDECAY [59,60], and PROPHECY4F [61,62], following the procedure discussed in Ref. [27]. The samples were produced with the ATLAS simulation infrastructure [63] using the full detector simulation performed by the GEANT4 [64] toolkit, with the exception of $b b H$ production of the MSSM Higgs boson signal, for which the ATLFASTII [63] fast simulation framework was used.

In this search, the leptonic $\tau$ decays are identified by their charged decay product, either an electron or a muon. Electron candidates are reconstructed from energy deposits in the electromagnetic calorimeter associated with a charged-particle track measured in the inner detector [93]. They are required to have $|\eta|<2.47$. The transition region between the barrel and end cap calorimeters $(1.37<|\eta|<1.52)$ is excluded.

Muon candidates are reconstructed in the range $|\eta|<2.5$ by matching tracks found in the muon spectrometer to tracks found in the inner detector [94]. The selected leptons in the $\tau_{\text {lep }} \tau_{\text {had }}$ channel are required to have a transverse momentum $p_{T}>30 \mathrm{GeV}$, pass the "medium" quality requirement for both the electrons [93] and muons [94] and satisfy a $p_{T^{-}}$and $\eta$-dependent isolation criterion called "Gradient", which uses calorimetric and tracking information. The efficiencies for the identification and isolation criteria are given in Refs. [93,94].

Jets are reconstructed from topological clusters [95] of energy depositions in the calorimeter using the anti- $k_{t}$ algorithm [96], with a radius parameter value $R=0.4$ [97]. The average energy contribution from pileup is subtracted according to the jet area and the jets are calibrated as described in Ref. [98]. Jets are required to have $p_{T}>$ $20 \mathrm{GeV}$ and $|\eta|<2.5$. The effect of pileup is reduced by using tracking information associated with the calorimeterbased jets to reject those not originating from the primary vertex [99]. The primary vertex is chosen as the protonproton vertex candidate with the highest sum of the squared transverse momenta of the associated tracks.

In order to identify jets containing $b$ hadrons ( $b$ jets), a multivariate algorithm (MV2) is used [100]. The algorithm has an average efficiency of $70 \%$ for $b$ jets and rejections of approximately 9, 36, and 300 for $c$ jets, $\tau$ decays with hadrons, and jets initiated by light quarks or gluons, respectively, as determined in simulated $t \bar{t}$ events. Correction factors are applied to the simulated event samples to compensate for differences between data and simulation in the $b$-tagging efficiencies for $b$ jets, $c$ jets and light-flavor jets.

Hadronic $\tau$ decays are composed of a neutrino and a set of visible decay products $\left(\tau_{\text {had-vis }}\right)$, typically one or three charged pions and up to two neutral pions. The $\tau_{\text {had-vis }}$ candidates reconstructed from seeding jets [101] must have $p_{T}>25(65) \mathrm{GeV}$ in the $\tau_{\text {lep }} \tau_{\text {had }}\left(\tau_{\text {had }} \tau_{\text {had }}\right)$ channel, $|\eta|<$ 2.5 excluding $1.37<|\eta|<1.52$, one or three associated tracks and an electric charge of \pm 1 . A boosted-decisiontree identification procedure, based on calorimetric shower shapes and tracking information, is used to reject jets.

The $\tau_{\text {had-vis }}$ candidates must satisfy "loose" or "medium" $\tau$ identification criteria [101] with efficiencies of about $85 \%$ (75\%) and $75 \%(60 \%)$ for one-track (three-track) $\tau_{\text {had-vis }}$ candidates, respectively. The rejections factors of "loose" and "medium" $\tau$ identification in multijet events are about 
20 (200) and 30 (500) for one-track (three-track) $\tau_{\text {had-vis }}$ candidates, respectively.

The missing transverse momentum, $\boldsymbol{E}_{T}^{\text {miss }}$, is calculated as the negative vectorial sum of the $\boldsymbol{p}_{T}$ of all fully reconstructed and calibrated physics objects [102]. In addition, this procedure includes a soft term, which is calculated using the inner-detector tracks that originate from the hard-scattering vertex but are not associated with reconstructed objects.

Events in the $\tau_{\text {lep }} \tau_{\text {had }}$ channel are selected using singleelectron and single-muon triggers with $p_{T}$ thresholds ranging from 20 to $26 \mathrm{GeV}$ and various isolation criteria $[103,104]$. The events must contain at least one $\tau_{\text {had-vis }}$ candidate passing the medium identification and exactly one isolated lepton $(\ell)$. The $\tau_{\text {had-vis }}$ candidate must have $|\eta|<2.3$ to reduce misidentified-electron background [105]. The isolated lepton and the $\tau_{\text {had-vis }}$ candidate must have opposite electric charge and be back to back in the transverse plane: $\left|\Delta \phi\left(\boldsymbol{p}_{T}^{\ell}, \boldsymbol{p}_{T}^{\tau_{\text {had-vis }}}\right)\right|>2.4 \mathrm{rad}$. To reduce background from $W+$ jets production the transverse mass $m_{T}\left(\boldsymbol{p}_{T}^{\ell}, \boldsymbol{E}_{T}^{\mathrm{miss}}\right)=\sqrt{2 p_{T}^{\ell} E_{T}^{\mathrm{miss}}\left[1-\cos \Delta \phi\left(\boldsymbol{p}_{T}^{\ell}, \boldsymbol{E}_{T}^{\mathrm{miss}}\right)\right]}, \quad$ calculated with the lepton $p_{T}$ and the event $\boldsymbol{E}_{T}^{\text {miss }}$, must be less than $40 \mathrm{GeV}$. To reduce background from $Z \rightarrow e e$ production in the $\tau_{e} \tau_{\text {had }}$ channel, events in which the isolated lepton and the $\tau_{\text {had-vis }}$ candidate have an invariant mass between 80 and $110 \mathrm{GeV}$ are rejected. The background contribution from $Z \rightarrow \mu \mu$ in the $\tau_{\mu} \tau_{\text {had }}$ channel is found to be negligible. The signal acceptance times efficiency for each of the $\tau_{e} \tau_{\text {had }}$ and $\tau_{\mu} \tau_{\text {had }}$ channels varies between $2 \%$ and $7 \%$ for signals with masses of $0.2-2.5 \mathrm{TeV}$ (the acceptance is calculated with respect to the sum of all $\tau$ decay modes; the efficiency is calculated taking into account detector acceptance, reconstruction and selection efficiencies).

Events in the $\tau_{\text {had }} \tau_{\text {had }}$ channel are selected by single- $\tau$ triggers with $p_{T}$ thresholds of $80 \mathrm{GeV}\left(5.4 \mathrm{fb}^{-1}\right.$ from June 2015 to May 2016), $125 \mathrm{GeV}$ (9.3 $\mathrm{fb}^{-1}$ in May-June 2016) and $160 \mathrm{GeV}$ (124 fb $\mathrm{fb}^{-1}$ from June 2016 to October 2018). Events must contain at least two $\tau_{\text {had-vis }}$ candidates and no electrons or muons. The $p_{T}$ of the leading $\tau_{\text {had-vis }}$ candidate must exceed the trigger $p_{T}$ threshold by $5 \mathrm{GeV}$. The leading (subleading) $\tau_{\text {had-vis }}$ candidate must satisfy the medium (loose) identification criteria. The two $\tau_{\text {had-vis }}$ must have opposite electric charge and be back to back in the transverse plane: $\left|\Delta \phi\left(\boldsymbol{p}_{T}^{\tau_{\text {had-vis }}^{1}}, \boldsymbol{p}_{T}^{\tau_{\text {had-vis }}^{2}}\right)\right|>2.7 \mathrm{rad}$. The signal acceptance times efficiency varies between $2 \%$ and $20 \%$ for signals with masses of $0.35-2.5 \mathrm{TeV}$, and it decreases rapidly for lower mass values due to the selection criteria imposed on the $p_{T}$ of the decay products of the $\tau$ leptons.

Events satisfying the selection criteria of either channel are divided into categories to exploit the different production modes in the MSSM: the $b$-tag category for events containing at least one $b$-jet and the $b$-veto category for events containing no $b$ jets. These categories are the signal regions used by the analysis.
The $\tau \tau$ mass reconstruction is crucial for good separation between signal and background events. However, its reconstruction is challenging due to the presence of neutrinos from the $\tau$-lepton decays. The mass reconstruction used for both channels is the total transverse mass, defined as $m_{T}^{\text {tot }}=\sqrt{\left(p_{T}^{\tau_{1}}+p_{T}^{\tau_{2}}+E_{T}^{\text {miss }}\right)^{2}-\left(\boldsymbol{p}_{T}^{\tau_{1}}+\boldsymbol{p}_{T}^{\tau_{2}}+\boldsymbol{E}_{T}^{\text {miss }}\right)^{2}}$ for either $\left(\ell, \tau_{\text {had-vis }}\right)$ or $\left(\tau_{\text {had-vis }}^{1}, \tau_{\text {had-vis }}^{2}\right)$ as $\left(\tau_{1}, \tau_{2}\right)$.

The dominant background contribution in the $\tau_{\text {lep }} \tau_{\text {had }}$ channel arises from processes where the $\tau_{\text {had-vis }}$ candidate originates from a jet. Such background events are divided into those where the selected lepton is correctly identified, mainly from $W+$ jets $(t \bar{t})$ production in the $b$-veto ( $b$-tag) category, and those where the selected lepton arises from a jet, mainly from multijet production. These contributions are estimated using a data-driven technique, which is similar to that described in Ref. [24]. Three orthogonal control regions are defined using the same selection as for the signal region, except that the lepton candidate fails isolation requirements in CR- 0 , the $\tau_{\text {had-vis }}$ candidate fails $\tau$ identification in CR-1, and both fail these conditions in CR2. The multijet background events are estimated from CR-0 weighted with lepton correction factors, called fake factors, which are ratios of the numbers of lepton candidates passing and failing the isolation requirements [24] (hereafter, fake factors refer to ratios of the number of candidates passing a certain identification requirement to the number of candidates failing the requirement). The $W+$ jets $(t \bar{t})$ background events are estimated from CR-1 after subtracting the multijet background contributions estimated from CR-2 corrected with lepton fake factors. Real $\tau$-lepton contributions in CR-1 are subtracted using MC simulation. The $\tau$-lepton fake-factor weights measured in data are then applied to the events in CR-1 to estimate the $W+$ jets $(t \bar{t})$ background in the signal region. Backgrounds where both the lepton and $\tau_{\text {had-vis }}$ candidates originate from electrons, muons or $\tau$ leptons arise from $Z / \gamma^{*} \rightarrow \tau \tau$ production in the $b$-veto category and $t \bar{t}$ production in the $b$-tag category,

TABLE II. Relative increase in the expected 95\% C.L. upper limits for the production cross section times branching fraction relative to the statistical only expected limit for each systematic uncertainty under consideration, shown for scalar bosons with mass of $400 \mathrm{GeV}$ and $1 \mathrm{TeV}$ produced via ggF and $b b H$ production.

\begin{tabular}{|c|c|c|c|c|}
\hline Source & $\begin{array}{c}g g F \\
(400 \mathrm{GeV})\end{array}$ & $\begin{array}{c}g g F \\
(1 \mathrm{TeV})\end{array}$ & $\begin{array}{c}b b H \\
(400 \mathrm{GeV})\end{array}$ & $\begin{array}{c}b b H \\
(1 \mathrm{TeV})\end{array}$ \\
\hline Tau id. efficiency & 0.14 & 0.16 & 0.12 & 0.08 \\
\hline Tau energy scale & 0.33 & 0.09 & 0.22 & 0.03 \\
\hline $\begin{array}{l}Z+\text { jets bkg. } \\
\text { modeling }\end{array}$ & 0.27 & 0.19 & 0.08 & 0.04 \\
\hline Mis-id. $\tau_{\text {had-vis }}$ bkg. & 0.22 & 0.01 & 0.14 & 0.03 \\
\hline Others & 0.09 & 0.04 & 0.11 & 0.02 \\
\hline Total & 0.54 & 0.28 & 0.45 & 0.13 \\
\hline
\end{tabular}


with minor contributions from $Z / \gamma^{*} \rightarrow \ell \ell$, diboson and single top-quark production. These contributions are estimated using MC simulation. To constrain the normalization of the $t \bar{t}$ contribution, a top-quark control region enhanced in $t \bar{t}$ events is defined by substituting the transverse mass requirement with $m_{T}\left(\boldsymbol{p}_{T}^{\ell}, \boldsymbol{E}_{T}^{\text {miss }}\right)>110$ (100) $\mathrm{GeV}$ in the $b$-tag category of the $\tau_{e} \tau_{\text {had }}\left(\tau_{\mu} \tau_{\text {had }}\right)$ channel. This region is included in the fitting procedure. Other major background contributions can be adequately constrained in the signal regions.

The dominant background contribution in the $\tau_{\text {had }} \tau_{\text {had }}$ channel is from multijet production, which is estimated using a data-driven technique described in Ref. [24]: the background is estimated from a control region whose events pass the same selection as for the signal region, except the subleading $\tau_{\text {had-vis }}$ candidates fail $\tau$ identification. Then the events are weighted with fake factors measured in a region enriched with multijet events to obtain the multijet background estimation in the signal region. The other nonnegligible backgrounds contributions are $Z / \gamma^{*} \rightarrow \tau \tau$ production in the $b$-veto category, $t \bar{t}$ production in the $b$ tag category, and to a lesser extent $W(\rightarrow \tau \nu, \ell \nu)+$ jets, single top-quark, diboson, and $Z / \gamma^{*}(\rightarrow \ell \ell)+$ jets production. These contributions are estimated using MC simulation. To improve the modeling of jets faking hadronic $\tau$ decays (fake $\tau$ leptons), events in the simulation that contain quark- or

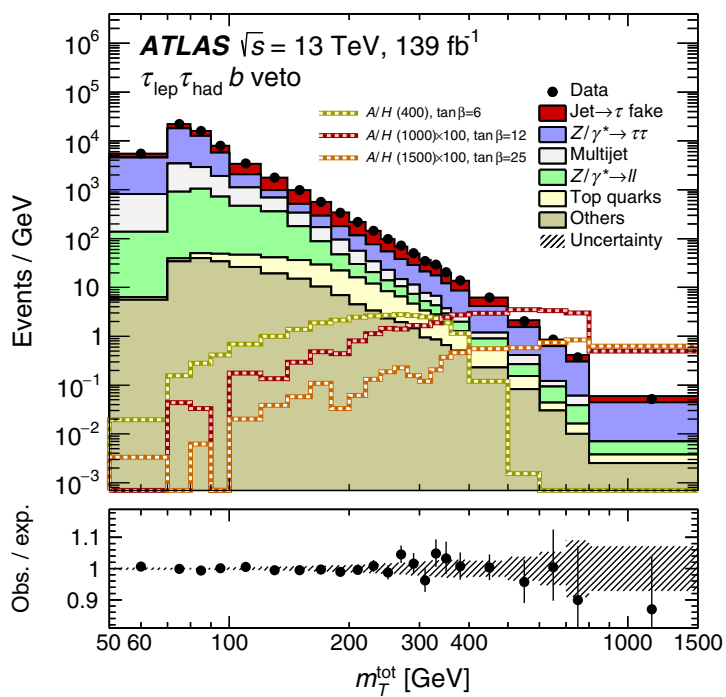

(a)

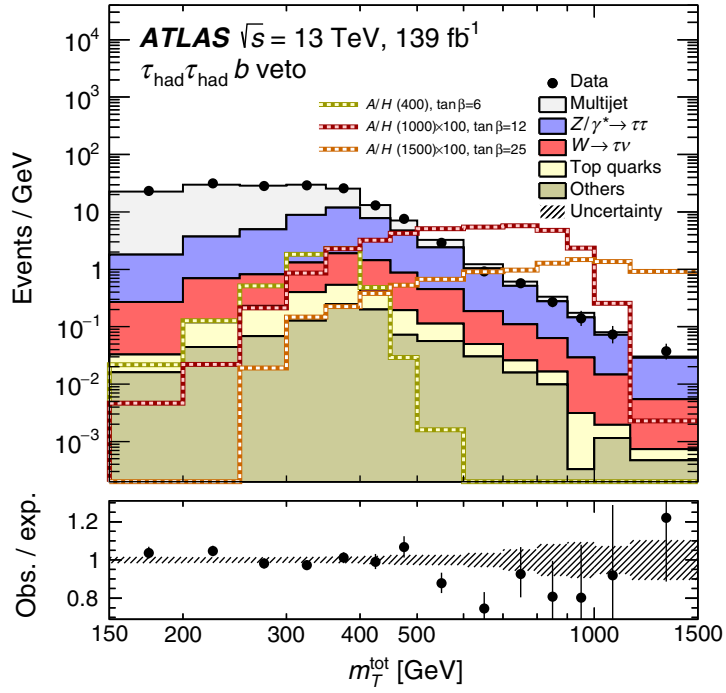

(c)

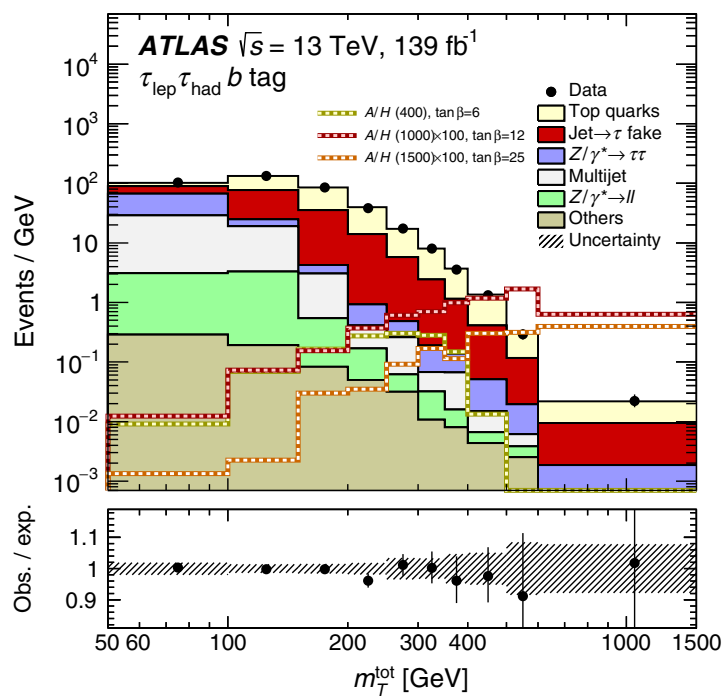

(b)

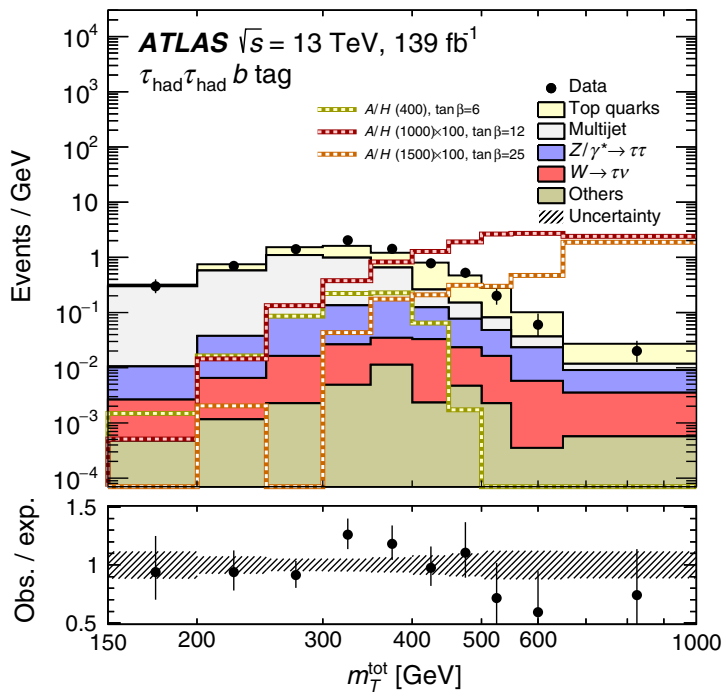

(d)

FIG. 1. The $m_{T}^{\text {tot }}$ for the $b$-veto (left) and $b$-tag (right) categories of the $\tau_{\text {lep }} \tau_{\text {had }}$ channel (top) and $\tau_{\text {had }} \tau_{\text {had }}$ channel (bottom). The binning displayed is that entering into the fit. The predictions and uncertainties for the background processes are obtained from the fit assuming the background-only hypothesis. Expectations from signal processes are superimposed. Overflows are included in the last bin of the distributions. 


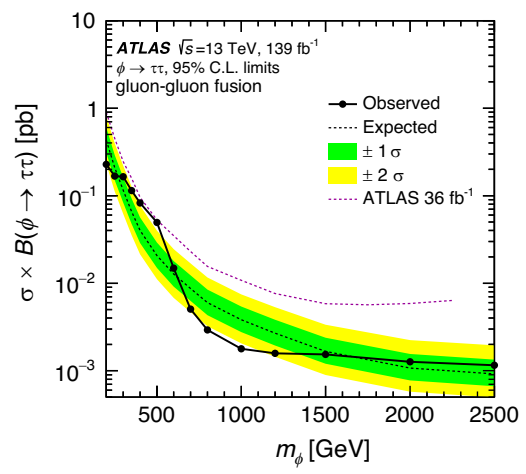

(a)

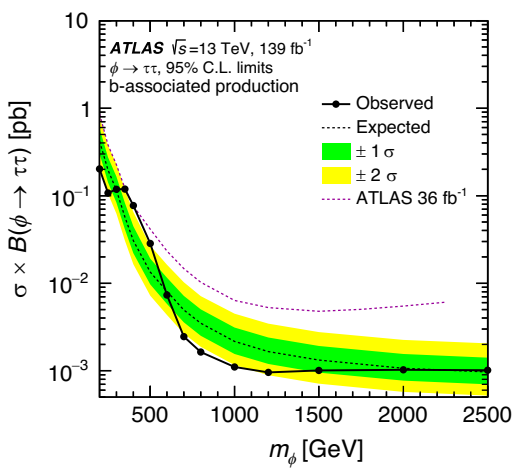

(b)

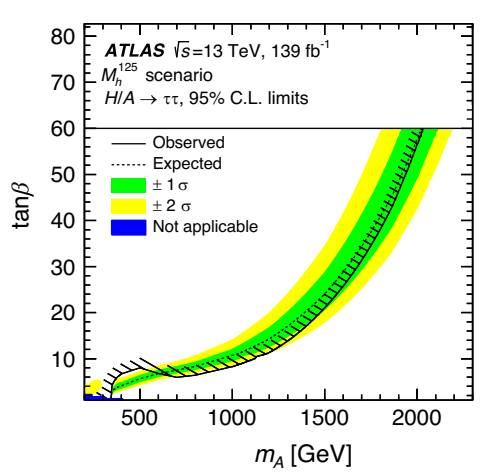

(c)

FIG. 2. The observed and expected 95\% C.L. upper limits on the production cross section times branching fraction for a scalar boson $(\phi)$ produced via (a) ggF and (b) $b$-associated production. The limits are calculated from a statistical combination of the $\tau_{\text {lep }} \tau_{\text {had }}$ and $\tau_{\text {had }} \tau_{\text {had }}$ channels. The excluded region from the 2015-2016 data ATLAS search [24] is depicted by the dotted pink line. The 95\% C.L. upper limits on $\tan \beta$ as a function of $m_{A}$ in the $M_{h}^{125}$ scenario is shown (c). The lowest value of $\tan \beta$ considered for the $M_{h}^{125}$ scenario is 0.5 . In the small lower-left region shown in solid blue, the mass splitting between $A$ and $H$ bosons is above $50 \%$ of the mass resolution and therefore the simple addition of the cross sections is not valid. However, this region of parameter space in the $M_{h}^{125}$ scenario provides predictions that are incompatible with the measured mass value of the observed Higgs boson by more than $3 \sigma$. The exclusion limit around $m_{A}=350 \mathrm{GeV}$ reflects the behavior of the $A \rightarrow \tau \tau$ branching fraction close to the $A \rightarrow t \bar{t}$ kinematic threshold for low tan $\beta$. The hatched area defines which side of the curve is excluded by the search.

gluon-initiated jets that are misidentified as $\tau_{\text {had-vis }}$ candidates are corrected to follow rates of fake $\tau$ leptons measured in $W+$ jets and $t \bar{t}$ enhanced regions in data.

Uncertainties affecting the simulated signal and background contributions are considered in the statistical analysis. These include uncertainties associated with the determination of the integrated luminosity [106,107], the detector simulation, the theoretical cross sections, and the background modeling. For MSSM Higgs boson samples, various sources of uncertainty which affect the signal acceptance are considered, such as the impact of varying the factorization and renormalization scales and uncertainties in the modeling of initial- and final-state radiation, as well as multiple parton interactions. The sensitivity of the search is limited by statistical uncertainties, especially for scalars with mass values above $600 \mathrm{GeV}$. The main systematic uncertainties are shown in Table II. They are related to the determination of the $\tau_{\text {had-vis }}$ identification efficiency and energy scale, estimation of the backgrounds with misidentified $\tau_{\text {had-vis }}$ and modeling of $Z+$ jets background. The uncertainty in the $\tau_{\text {had-vis }}$ identification efficiency is determined from measurements of $Z \rightarrow \tau \tau$ events and, for the high $p_{T}$ regime, an additional uncertainty is assigned from the validation of the $\tau_{\text {had-vis }}$ properties in high- $p_{T}$ dijet events. The uncertainty in the $\tau_{\text {had-vis }}$ energy scale is derived from $Z \rightarrow \tau \tau$ events as well, and from single hadron test-beam data, and it is validated for high- $p_{T}$ $\tau_{\text {had-vis }}$ with top-quark events and $Z(\rightarrow \tau \tau)$ events with large transverse momentum. Uncertainties in the determination of backgrounds with misidentified $\tau_{\text {had-vis }}$ include the uncertainty from the subtraction of other backgrounds in the control regions, the uncertainty from the limited number of events in the control regions and the uncertainty from differences in the jet composition between control regions and signal regions. For $Z+$ jets production, cross-section and modeling uncertainties are taken from Refs. [108,109].

A simultaneous fit of the $m_{T}^{\text {tot }}$ distributions of the topquark control region and of the $b$-veto and $b$-tag categories of the $\tau_{\text {lep }} \tau_{\text {had }}$ and $\tau_{\text {had }} \tau_{\text {had }}$ channels is performed in the statistical analysis. The numbers of observed events in the $b$-veto and $b$-tag categories of the $\tau_{\text {lep }} \tau_{\text {had }}$ channel are 728 174 and 19542 , while event yields of $728200 \pm 2900$ and $19600 \pm 400$ for the background-only hypothesis are obtained from the statistical analysis, which includes the fit of the nuisance parameters associated with the systematic uncertainties.

For the $\tau_{\text {had }} \tau_{\text {had }}$ channel, the numbers of observed events in the $b$-veto and $b$-tag categories are 8420 and 381, and the fitted event yields from background processes are $8430 \pm$ 150 and $368 \pm 27$. The $m_{T}^{\text {tot }}$ distributions obtained from the fit performed simultaneously in the $b$-veto and $b$-tag categories of the two channels are shown in Fig. 1.

The data are found to be in good agreement with the obtained background yields, and the results are given in terms of exclusion limits. Upper limits on the cross section times branching fraction for a scalar boson (generically called $\phi$ ) decaying into $\tau$-lepton pairs are set at the 95\% confidence level (C.L.) as a function of the boson mass. They are computed using a modified frequentist CLs method [110] with the profile likelihood ratio as the test statistic. The asymptotic approximation is used [111]. The upper limits cover the mass range $0.2-2.5 \mathrm{TeV}$ and are shown for a production entirely via ggF in Fig. 2(a) and entirely via $b$-quark associated production in Fig. 2(b). The observed (expected) upper limits are $1.8 \mathrm{fb}$ (3.8 fb) for $\mathrm{ggF}$ and $1.1 \mathrm{fb}(2.2 \mathrm{fb})$ for $b b H$ production at $m_{\phi}=1 \mathrm{TeV}$. For 
ggF, the lowest local $p_{0}$, the probability that the background can produce a fluctuation greater than the excess observed in data, is $0.014(2.2 \sigma)$ at $m_{\phi}=400 \mathrm{GeV}$, while for $b b H$ production it is $0.003(2.7 \sigma)$ at $m_{\phi}=400 \mathrm{GeV}$. The natural width of the scalar boson is assumed to be negligible compared to the experimental resolution. Results are interpreted in terms of the MSSM in Fig. 2(c), which shows the regions in the $m_{A}-\tan \beta$ plane excluded at the 95\% C.L. in the $M_{h}^{125}$ scenario. The observed (expected) upper limits exclude $\tan \beta>21$ (24) for $m_{A}=1.5 \mathrm{TeV}$.

In conclusion, a search for heavy neutral Higgs bosons decaying into a pair of $\tau$ leptons is performed in the mass range $0.2-2.5 \mathrm{TeV}$ using a data sample corresponding to an integrated luminosity of $139 \mathrm{fb}^{-1}$ from proton-proton collisions at $\sqrt{s}=13 \mathrm{TeV}$ recorded by the ATLAS detector at the LHC. No significant excess over the expected SM backgrounds is found. Upper limits on the cross section for the production of a scalar boson times the branching fraction to $\tau \tau$ final states are set at the 95\% C.L., significantly increasing the sensitivity and explored mass range compared to previous searches. They are in the range 240$1.2 \mathrm{fb}(230-1.0 \mathrm{fb})$ for gluon-gluon fusion ( $b$-associated) production of scalar bosons with masses of $0.2-2.5 \mathrm{TeV}$. In the $M_{h}^{125}$ scenario, the data exclude $\tan \beta>8$ for $m_{A}=$ $1.0 \mathrm{TeV}$ and $\tan \beta>21$ for $m_{A}=1.5 \mathrm{TeV}$ at the $95 \%$ C.L.

We thank CERN for the very successful operation of the LHC, as well as the support staff from our institutions without whom ATLAS could not be operated efficiently. We acknowledge the support of ANPCyT, Argentina; YerPhI, Armenia; ARC, Australia; BMWFW and FWF, Austria; ANAS, Azerbaijan; SSTC, Belarus; CNPq and FAPESP, Brazil; NSERC, NRC and CFI, Canada; CERN; CONICYT, Chile; CAS, MOST and NSFC, China; COLCIENCIAS, Colombia; MSMT CR, MPO CR and VSC CR, Czech Republic; DNRF and DNSRC, Denmark; IN2P3-CNRS and CEA-DRF/IRFU, France; SRNSFG, Georgia; BMBF, HGF and MPG, Germany; GSRT, Greece; RGC and Hong Kong SAR, China; ISF and Benoziyo Center, Israel; INFN, Italy; MEXT and JSPS, Japan; CNRST, Morocco; NWO, Netherlands; RCN, Norway; MNiSW and NCN, Poland; FCT, Portugal; MNE/IFA, Romania; MES of Russia and NRC KI, Russia Federation; JINR; MESTD, Serbia; MSSR, Slovakia; ARRS and MIZŠ, Slovenia; DST/NRF, South Africa; MINECO, Spain; SRC and Wallenberg Foundation, Sweden; SERI, SNSF and Cantons of Bern and Geneva, Switzerland; MOST, Taiwan; TAEK, Turkey; STFC, United Kingdom; DOE and NSF, United States of America. In addition, individual groups and members have received support from BCKDF, CANARIE, Compute Canada and CRC, Canada; ERC, ERDF, Horizon 2020, Marie Skłodowska-Curie Actions and COST, European Union; Investissements d'Avenir Labex, Investissements d'Avenir Idex and ANR, France; DFG and AvH
Foundation, Germany; Herakleitos, Thales and Aristeia programmes co-financed by EU-ESF and the Greek NSRF, Greece; BSF-NSF and GIF, Israel; CERCA Programme Generalitat de Catalunya and PROMETEO Programme Generalitat Valenciana, Spain; Göran Gustafssons Stiftelse, Sweden; The Royal Society and Leverhulme Trust, United Kingdom. The crucial computing support from all WLCG partners is acknowledged gratefully, in particular from CERN, the ATLAS Tier-1 facilities at TRIUMF (Canada), NDGF (Denmark, Norway, Sweden), CC-IN2P3 (France), KIT/GridKA (Germany), INFNCNAF (Italy), NL-T1 (Netherlands), PIC (Spain), ASGC (Taiwan), RAL (UK), and BNL (USA), the Tier-2 facilities worldwide and large non-WLCG resource providers. Major contributors of computing resources are listed in Ref. [112].

[1] ATLAS Collaboration, Observation of a new particle in the search for the Standard Model Higgs boson with the ATLAS detector at the LHC, Phys. Lett. B 716, 1 (2012).

[2] CMS Collaboration, Observation of a new boson at a mass of $125 \mathrm{GeV}$ with the CMS experiment at the LHC, Phys. Lett. B 716, 30 (2012).

[3] ATLAS Collaboration, Combined measurements of Higgs boson production and decay using up to $80 \mathrm{fb}^{-1}$ of protonproton collision data at $\sqrt{s}=13 \mathrm{TeV}$ collected with the ATLAS experiment, Phys. Rev. D 101, 012002 (2020).

[4] CMS Collaboration, Combined measurements of Higgs boson couplings in proton-proton collisions at $\sqrt{s}=13 \mathrm{TeV}$, Eur. Phys. J. C 79, 421 (2019).

[5] F. Englert and R. Brout, Broken Symmetry and the Mass of Gauge Vector Mesons, Phys. Rev. Lett. 13, 321 (1964).

[6] P. W. Higgs, Broken Symmetries and the Masses of Gauge Bosons, Phys. Rev. Lett. 13, 508 (1964).

[7] G. S. Guralnik, C. R. Hagen, and T. W. B. Kibble, Global Conservation Laws and Massless Particles, Phys. Rev. Lett. 13, 585 (1964).

[8] G. C. Branco, P. M. Ferreira, L. Lavoura, M. N. Rebelo, M. Sher, and J. P. Silva, Theory and phenomenology of twoHiggs-doublet models, Phys. Rep. 516, 1 (2012).

[9] P. Fayet, Supersymmetry and weak, electromagnetic and strong interactions, Phys. Lett. 64B, 159 (1976).

[10] P. Fayet, Spontaneously broken supersymmetric theories of weak, electromagnetic and strong interactions, Phys. Lett. 69B, 489 (1977).

[11] Y. A. Golfand and E. P. Likhtman, Extension of the algebra of Poincare group generators and violation of $p$ invariance, Pis'ma Zh. Eksp. Teor. Fiz. 13, 452 (1971) [JETP Lett. 13, 323 (1971)].

[12] D. V. Volkov and V. P. Akulov, Is the neutrino a goldstone particle?, Phys. Lett. 46B, 109 (1973).

[13] J.Wess and B. Zumino, Supergauge transformations in four dimensions, Nucl. Phys. B70, 39 (1974).

[14] A. Salam and J. Strathdee, Super-symmetry and nonAbelian gauges, Phys. Lett. 51B, 353 (1974).

[15] J. Wess and B. Zumino, Supergauge invariant extension of quantum electrodynamics, Nucl. Phys. B78, 1 (1974). 
[16] S. Ferrara and B. Zumino, Supergauge invariant YangMills theories, Nucl. Phys. B79, 413 (1974).

[17] E. Bagnaschi et al., MSSM Higgs boson searches at the LHC: Benchmark scenarios for Run 2 and beyond, Eur. Phys. J. C 79, 617 (2019).

[18] L. Evans and P. Bryant, LHC machine, J. Instrum. 3, S08001 (2008).

[19] ATLAS Collaboration, The ATLAS experiment at the CERN large Hadron collider, J. Instrum. 3, S08003 (2008).

[20] ATLAS Collaboration, ATLAS insertable B-layer technical design report, CERN Report No. ATLAS-TDR-19, 2010, https://cds.cern.ch/record/1291633; Addendum, CERN Report No. ATLAS-TDR-19-ADD-1, 2012, https://cds.cern .ch/record/1451888.

[21] B. Abbott et al., Production and integration of the ATLAS insertable B-layer, J. Instrum. 13, T05008 (2018).

[22] ATLAS Collaboration, ATLAS data quality operations and performance for 2015-2018 data-taking, J. Instrum. 15, P04003 (2020).

[23] ATLAS Collaboration, Search for minimal supersymmetric standard model Higgs bosons $H / A$ and for a $Z^{\prime}$ boson in the $\tau \tau$ final state produced in $p p$ collisions at $\sqrt{s}=$ $13 \mathrm{TeV}$ with the ATLAS detector, Eur. Phys. J. C 76, 585 (2016).

[24] ATLAS Collaboration, Search for additional heavy neutral Higgs and gauge bosons in the ditau final state produced in $36 \mathrm{fb}^{-1}$ of $p p$ collisions at $\sqrt{s}=13 \mathrm{TeV}$ with the ATLAS detector, J. High Energy Phys. 01 (2018) 055.

[25] CMS Collaboration, Search for additional neutral MSSM Higgs bosons in the $\tau \tau$ final state in proton-proton collisions at $\sqrt{s}=13 \mathrm{TeV}$, J. High Energy Phys. 09 (2018) 007.

[26] ATLAS uses a right-handed coordinate system with its origin at the nominal interaction point (IP) in the center of the detector and the $z$ axis along the beam pipe. The $x$ axis points from the IP to the center of the LHC ring, and the $y$ axis points upward. Cylindrical coordinates $(r, \phi)$ are used in the transverse plane, $\phi$ being the azimuthal angle around the $z$ axis. The pseudorapidity is defined in terms of the polar angle $\theta$ as $\eta=-\ln \tan (\theta / 2)$.

[27] LHC Higgs Cross Section Working Group, Handbook of LHC Higgs cross sections: 1. Inclusive observables, https://doi.org/10.5170/CERN-2011-002 (2011).

[28] LHC Higgs Cross Section Working Group, Handbook of LHC Higgs cross sections: 4. Deciphering the nature of the Higgs sector, https://doi.org/https://dx.doi.org/10.23731/ CYRM-2017-002 (2016).

[29] R. V. Harlander, S. Liebler, and H. Mantler, SusHi: A program for the calculation of Higgs production in gluon fusion and bottom-quark annihilation in the Standard Model and the MSSM, Comput. Phys. Commun. 184, 1605 (2013).

[30] R. V. Harlander, S. Liebler, and H. Mantler, SusHi Bento: Beyond NNLO and the heavy-top limit, Comput. Phys. Commun. 212, 239 (2017).

[31] M. Spira, A. Djouadi, D. Graudenz, and P. M. Zerwas, Higgs boson production at the LHC, Nucl. Phys. B453, 17 (1995).

[32] R. V. Harlander and M. Steinhauser, Supersymmetric Higgs production in gluon fusion at next-toleading order, J. High Energy Phys. 09 (2004) 066.
[33] R. V. Harlander and P. Kant, Higgs production and decay: Analytic results at next-to-leading order QCD, J. High Energy Phys. 12 (2005) 015.

[34] G. Degrassi and P. Slavich, NLO QCD bottom corrections to Higgs boson production in the MSSM, J. High Energy Phys. 11 (2010) 044.

[35] G. Degrassi, S. Di Vita, and P. Slavich, NLO QCD corrections to pseudoscalar Higgs production in the MSSM, J. High Energy Phys. 08 (2011) 128.

[36] G. Degrassi, S. Di Vita, and P. Slavich, On the NLO QCD corrections to the production of the heaviest neutral Higgs scalar in the MSSM, Eur. Phys. J. C 72, 2032 (2012).

[37] R. V. Harlander and W. B. Kilgore, Next-to-Next-to-Leading Order Higgs Production at Hadron Colliders, Phys. Rev. Lett. 88, 201801 (2002).

[38] C. Anastasiou and K. Melnikov, Higgs boson production at hadron colliders in NNLO QCD, Nucl. Phys. B646, 220 (2002).

[39] V. Ravindran, J. Smith, and W. L. van Neerven, NNLO corrections to the total cross section for Higgs boson production in hadron-hadron collisions, Nucl. Phys. B665, 325 (2003).

[40] R. V. Harlander and W. B. Kilgore, Production of a pseudo-scalar Higgs boson at hadron colliders at nextto-next-to-leading order, J. High Energy Phys. 10 (2002) 017.

[41] C. Anastasiou and K. Melnikov, Pseudoscalar Higgs boson production at hadron colliders in next-to-next-to-leading order QCD, Phys. Rev. D 67, 037501 (2003).

[42] U. Aglietti, R. Bonciani, G. Degrassi, and A. Vicini, Twoloop light fermion contribution to Higgs production and decays, Phys. Lett. B 595, 432 (2004).

[43] R. Bonciani, G. Degrassi, and A. Vicini, On the generalized harmonic polylogarithms of one complex variable, Comput. Phys. Commun. 182, 1253 (2011).

[44] R. V. Harlander and W. B. Kilgore, Higgs boson production in bottom quark fusion at next-to-next-to-leading order, Phys. Rev. D 68, 013001 (2003).

[45] S. Dittmaier, M. Krämer, and M. Spira, Higgs radiation off bottom quarks at the Fermilab Tevatron and the CERN LHC, Phys. Rev. D 70, 074010 (2004).

[46] S. Dawson, C. B. Jackson, L. Reina, and D. Wackeroth, Exclusive Higgs boson production with bottom quarks at hadron colliders, Phys. Rev. D 69, 074027 (2004).

[47] M. Bonvini, A. S. Papanastasiou, and F. J. Tackmann, Resummation and matching of $b$-quark mass effects in $b \bar{b} H$ production, J. High Energy Phys. 11 (2015) 196.

[48] M. Bonvini, A. S. Papanastasiou, and F. J. Tackmann, Matched predictions for the $b \bar{b} H$ cross section at the 13 TeV LHC, J. High Energy Phys. 10 (2016) 053.

[49] S. Forte, D. Napoletano, and M. Ubiali, Higgs production in bottom-quark fusion in a matched scheme, Phys. Lett. B 751, 331 (2015).

[50] S. Forte, D. Napoletano, and M. Ubiali, Higgs production in bottom-quark fusion: Matching beyond leading order, Phys. Lett. B 763, 190 (2016).

[51] S. Heinemeyer, W. Hollik, and G. Weiglein, FeynHiggs: A program for the calculation of the masses of the neutral CP-even Higgs bosons in the MSSM, Comput. Phys. Commun. 124, 76 (2000). 
[52] S. Heinemeyer, W. Hollik, and G. Weiglein, The masses of the neutral $C P$-even Higgs bosons in the MSSM: Accurate analysis at the two-loop level, Eur. Phys. J. C 9, 343 (1999).

[53] G. Degrassi, S. Heinemeyer, W. Hollik, P. Slavich, and G. Weiglein, Towards high-precision predictions for the MSSM Higgs sector, Eur. Phys. J. C 28, 133 (2003).

[54] M. Frank, T. Hahn, S. Heinemeyer, W. Hollik, H. Rzehak, and G. Weiglein, The Higgs boson masses and mixings of the complex MSSM in the Feynman-diagrammatic approach, J. High Energy Phys. 02 (2007) 047.

[55] T. Hahn, S. Heinemeyer, W. Hollik, H. Rzehak, and G. Weiglein, High-Precision Predictions for the Light $C P$ Even Higgs Boson Mass of the Minimal Supersymmetric Standard Model, Phys. Rev. Lett. 112, 141801 (2014).

[56] K. E. Williams, H. Rzehak, and G. Weiglein, Higher-order corrections to Higgs boson decays in the MSSM with complex parameters, Eur. Phys. J. C 71, 1669 (2011).

[57] H. Bahl and W. Hollik, Precise prediction for the light MSSM Higgs-boson mass combining effective field theory and fixed-order calculations, Eur. Phys. J. C 76, 499 (2016).

[58] H. Bahl, S. Heinemeyer, W. Hollik, and G. Weiglein, Reconciling EFT and hybrid calculations of the light MSSM Higgs-boson mass, Eur. Phys. J. C 78, 57 (2018).

[59] A. Djouadi, J. Kalinowski, and M. Spira, HDECAY: A program for Higgs boson decays in the Standard Model and its supersymmetric extension, Comput. Phys. Commun. 108, 56 (1998).

[60] A. Djouadi, J. Kalinowski, M. Müehlleitner, and M. Spira, HDECAY: Twenty++ years after, Comput. Phys. Commun. 238, 214 (2019).

[61] A. Bredenstein, A. Denner, S. Dittmaier, and M. M. Weber, Precise predictions for the Higgs-boson decay $H \rightarrow$ $W W / Z Z \rightarrow 4$ leptons, Phys. Rev. D 74, 013004 (2006).

[62] A. Bredenstein, A. Denner, S. Dittmaier, and W. M. Weber, Radiative corrections to the semileptonic and hadronic Higgs-boson decays $\mathrm{H} \rightarrow \mathrm{WW} / \mathrm{ZZ} \rightarrow 4$ fermions, J. High Energy Phys. 02 (2007) 080.

[63] ATLAS Collaboration, The ATLAS simulation infrastructure, Eur. Phys. J. C 70, 823 (2010).

[64] S. Agostinelli et al., GEANT4-A simulation toolkit, Nucl. Instrum. Methods Phys. Res., Sect. A 506, 250 (2003).

[65] P. Nason, A new method for combining NLO QCD with shower Monte Carlo algorithms, J. High Energy Phys. 11 (2004) 040.

[66] S. Frixione, P. Nason, and C. Oleari, Matching NLO QCD computations with parton shower simulations: The POWHEG method, J. High Energy Phys. 11 (2007) 070.

[67] S. Alioli, P. Nason, C. Oleari, and E. Re, A general framework for implementing NLO calculations in shower Monte Carlo programs: The POWHEG BOX, J. High Energy Phys. 06 (2010) 043.

[68] S. Alioli, P. Nason, C. Oleari, and E. Re, NLO Higgs boson production via gluon fusion matched with shower in POWHEG, J. High Energy Phys. 04 (2009) 002.

[69] E. Bagnaschi, G. Degrassi, P. Slavich, and A. Vicini, Higgs production via gluon fusion in the POWHEG approach in the SM and in the MSSM, J. High Energy Phys. 02 (2012) 088.
[70] H.-L. Lai, M. Guzzi, J. Huston, Z. Li, P. M. Nadolsky, J. Pumplin, and C. P. Yuan, New parton distributions for collider physics, Phys. Rev. D 82, 074024 (2010).

[71] T. Sjöstrand, S. Mrenna, and P. Skands, A brief introduction to Pythia 8.1, Comput. Phys. Commun. 178, 852 (2008).

[72] J. Alwall, R. Frederix, S. Frixione, V. Hirschi, F. Maltoni, O. Mattelaer, H.-S. Shao, T. Stelzer, P. Torrielli, and M. Zaro, The automated computation of tree-level and nextto-leading order differential cross sections, and their matching to parton shower simulations, J. High Energy Phys. 07 (2014) 079.

[73] M. Wiesemann, R. Frederix, S. Frixione, V. Hirschi, F. Maltoni, and P. Torrielli, Higgs production in association with bottom quarks, J. High Energy Phys. 02 (2015) 132.

[74] T. Sjöstrand, S. Ask, J. R. Christiansen, R. Corke, N. Desai, P. Ilten, S. Mrenna, S. Prestel, C. O. Rasmussen, and P. Z. Skands, An introduction to PYTHia 8.2, Comput. Phys. Commun. 191, 159 (2015).

[75] E. Bothmann et al., Event generation with Sherpa 2.2, SciPost Phys. 7, 034 (2019).

[76] R. D. Ball et al., Parton distributions for the LHC run II, J. High Energy Phys. 04 (2015) 040.

[77] S. Schumann and F. Krauss, A parton shower algorithm based on Catani-Seymour dipole factorisation, J. High Energy Phys. 03 (2008) 038.

[78] C. Anastasiou, L. Dixon, K. Melnikov, and F. Petriello, High-precision QCD at hadron colliders: Electroweak gauge boson rapidity distributions at next-to-next-to leading order, Phys. Rev. D 69, 094008 (2004).

[79] S. Alioli, P. Nason, C. Oleari, and E. Re, NLO vectorboson production matched with shower in POWHEG, J. High Energy Phys. 07 (2008) 060.

[80] S. Frixione, P. Nason, and G. Ridolfi, A positive-weight next-to-leading-order Monte Carlo for heavy flavour hadroproduction, J. High Energy Phys. 09 (2007) 126.

[81] M. Beneke, P. Falgari, S. Klein, and C. Schwinn, Hadronic top-quark pair production with NNLL threshold resummation, Nucl. Phys. B855, 695 (2012).

[82] M. Cacciari, M. Czakon, M. Mangano, A. Mitov, and P. Nason, Top-pair production at hadron colliders with nextto-next-to-leading logarithmic soft-gluon resummation, Phys. Lett. B 710, 612 (2012).

[83] P. Bärnreuther, M. Czakon, and A. Mitov, PercentLevel-Precision Physics at the Tevatron: Next-to-Nextto-Leading Order QCD Corrections to $q \bar{q} \rightarrow t \bar{t}+X$, Phys. Rev. Lett. 109, 132001 (2012).

[84] M. Czakon and A. Mitov, NNLO corrections to top-pair production at hadron colliders: The all-fermionic scattering channels, J. High Energy Phys. 12 (2012) 054.

[85] M. Czakon and A. Mitov, NNLO corrections to top pair production at hadron colliders: The quark-gluon reaction, J. High Energy Phys. 01 (2013) 080.

[86] M. Czakon, P. Fiedler, and A. Mitov, Total Top-Quark PairProduction Cross Section at Hadron Colliders Through $O\left(\alpha_{S}^{4}\right)$, Phys. Rev. Lett. 110, 252004 (2013).

[87] M. Czakon and A. Mitov, Top++: A program for the calculation of the top-pair cross-section at hadron colliders, Comput. Phys. Commun. 185, 2930 (2014). 
[88] E. Re, Single-top Wt-channel production matched with parton showers using the POWHEG method, Eur. Phys. J. C 71, 1547 (2011).

[89] R. Frederix, E. Re, and P. Torrielli, Single-top $t$-channel hadroproduction in the four-flavour scheme with POWHEG and aMC@NLO, J. High Energy Phys. 09 (2012) 130.

[90] S. Alioli, P. Nason, C. Oleari, and E. Re, NLO singletop production matched with shower in POWHEG: $s$ - and $t$-channel contributions, J. High Energy Phys. 09 (2009) 111; Erratum, J. High Energy Phys. 02 (2010) 11.

[91] M. Aliev, H. Lacker, U. Langenfeld, S. Moch, P. Uwer, and M. Wiedermann, HATHOR: HAdronic Top and Heavy quarks crOss section calculatoR, Comput. Phys. Commun. 182, 1034 (2011).

[92] P. Kant, O. M. Kind, T. Kintscher, T. Lohse, T. Martini, S. Mölbitz, P. Rieck, and P. Uwer, HATHOR for single topquark production: Updated predictions and uncertainty estimates for single top-quark production in hadronic collisions, Comput. Phys. Commun. 191, 74 (2015).

[93] ATLAS Collaboration, Electron and photon performance measurements with the ATLAS detector using the 20152017 LHC proton-proton collision data, J. Instrum. 14, P12006 (2019).

[94] ATLAS Collaboration, Muon reconstruction performance of the ATLAS detector in proton-proton collision data at $\sqrt{s}=13 \mathrm{TeV}$, Eur. Phys. J. C 76, 292 (2016).

[95] ATLAS Collaboration, Topological cell clustering in the ATLAS calorimeters and its performance in LHC Run 1, Eur. Phys. J. C 77, 490 (2017).

[96] M. Cacciari, G. P. Salam, and G. Soyez, The anti- $k_{t}$ jet clustering algorithm, J. High Energy Phys. 04 (2008) 063.

[97] M. Cacciari, G. P. Salam, and G. Soyez, FastJet user manual, Eur. Phys. J. C 72, 1896 (2012).

[98] ATLAS Collaboration, Jet energy scale measurements and their systematic uncertainties in proton-proton collisions at $\sqrt{s}=13 \mathrm{TeV}$ with the ATLAS detector, Phys. Rev. D 96, 072002 (2017).

[99] ATLAS Collaboration, Performance of pile-up mitigation techniques for jets in $p p$ collisions at $\sqrt{s}=8 \mathrm{TeV}$ using the ATLAS detector, Eur. Phys. J. C 76, 581 (2016).

[100] ATLAS Collaboration, ATLAS $b$-jet identification performance and efficiency measurement with $t \bar{t}$ events in pp collisions at $\sqrt{s}=13 \mathrm{TeV}$, Eur. Phys. J. C 79, 970 (2019).

[101] ATLAS Collaboration, Measurement of the tau lepton reconstruction and identification performance in the ATLAS experiment using $p p$ collisions at $\sqrt{s}=13 \mathrm{TeV}, \mathrm{CERN}$ Report No. ATLAS-CONF-2017-029, 2017, https://cds .cern.ch/record/2261772.

[102] ATLAS Collaboration, $E_{T}^{\text {miss }}$ performance in the ATLAS detector using 2015-2016 LHC $p p$ collisions, CERN Report No. ATLAS-CONF-2018-023, 2018, https://cds.cern.ch/ record/2625233.

[103] ATLAS Collaboration, Performance of electron and photon triggers in ATLAS during LHC Run 2, Eur. Phys. J. C 80, 47 (2020).

[104] ATLAS Collaboration, Performance of the ATLAS trigger system in 2015, Eur. Phys. J. C 77, 317 (2017).

[105] ATLAS Collaboration, Identification and energy calibration of hadronically decaying tau leptons with the ATLAS experiment in $p p$ collisions at $\sqrt{s}=8 \mathrm{TeV}$, Eur. Phys. J. C 75, 303 (2015).

[106] G. Avoni et al., The new LUCID-2 detector for luminosity measurement and monitoring in ATLAS, J. Instrum. 13, P07017 (2018).

[107] ATLAS Collaboration, Luminosity determination in $p p$ collisions at $\sqrt{s}=13 \mathrm{TeV}$ using the ATLAS detector at the LHC, CERN Report No. ATLAS-CONF-2019-021, 2019, https://cds.cern.ch/record/2677054.

[108] ATLAS Collaboration, Search for high-mass new phenomena in the dilepton final state using proton-proton collisions at $\sqrt{s}=13 \mathrm{TeV}$ with the ATLAS detector, Phys. Lett. B 761, 372 (2016).

[109] J. R. Andersen et al., Les Houches 2013: Physics at TeV colliders: Standard Model working group report, arXiv: 1405.1067.

[110] A. L. Read, Presentation of search results: The CLs technique, J. Phys. G 28, 2693 (2002).

[111] G. Cowan, K. Cranmer, E. Gross, and O. Vitells, Asymptotic formulae for likelihood-based tests of new physics, Eur. Phys. J. C 71, 1554 (2011); Erratum, Eur. Phys. J. C 73, 2501 (2013).

[112] ATLAS Collaboration, ATLAS computing acknowledgements, CERN Report No. ATL-GEN-PUB-2016-002, https://cds.cern.ch/record/2202407.

G. Aad, ${ }^{102}$ B. Abbott, ${ }^{128}$ D. C. Abbott, ${ }^{103}$ A. Abed Abud, ${ }^{36}$ K. Abeling, ${ }^{53}$ D. K. Abhayasinghe, ${ }^{94}$ S. H. Abidi, ${ }^{166}$ O. S. AbouZeid, ${ }^{40}$ N. L. Abraham, ${ }^{155}$ H. Abramowicz, ${ }^{160}$ H. Abreu, ${ }^{159}$ Y. Abulaiti, ${ }^{6}$ B. S. Acharya, ${ }^{67,67 b, b}$ B. Achkar, ${ }^{53}$ L. Adam, ${ }^{100}$ C. Adam Bourdarios, ${ }^{5}$ L. Adamczyk, ${ }^{84 a}$ L. Adamek, ${ }^{166}$ J. Adelman, ${ }^{121}$ M. Adersberger, ${ }^{114}$ A. Adiguzel, ${ }^{12 c}$ S. Adorni ${ }^{54}$ T. Adye ${ }^{143}$ A. A. Affolder, ${ }^{145}$ Y. Afik, ${ }^{159}$ C. Agapopoulou,${ }^{65}$ M. N. Agaras, ${ }^{38}$ A. Aggarwal, ${ }^{119}$ C. Agheorghiesei, ${ }^{27 \mathrm{c}}$ J. A. Aguilar-Saavedra, ${ }^{139 f, 139 a, \mathrm{c}}$ A. Ahmad ${ }^{36}$ F. Ahmadov, ${ }^{80}$ W. S. Ahmed,${ }^{104}$ X. Ai,${ }^{18}$ G. Aielli, ${ }^{74 a, 74 b}$ S. Akatsuka, ${ }^{86}$ T. P. A. Åkesson, ${ }^{97}$ E. Akilli, ${ }^{54}$ A. V. Akimov, ${ }^{111}$ K. Al Khoury, ${ }^{65}$ G. L. Alberghi, ${ }^{23 b, 23 a}$ J. Albert, ${ }^{175}$ M. J. Alconada Verzini, ${ }^{160}$ S. Alderweireldt, ${ }^{36}$ M. Aleksa ${ }^{36}$ I. N. Aleksandrov, ${ }^{80}$ C. Alexa, ${ }^{27 b}$ T. Alexopoulos,${ }^{10}$ A. Alfonsi, ${ }^{120}$ F. Alfonsi, ${ }^{23 b, 23 a}$ M. Alhroob, ${ }^{128}$ B. Ali, ${ }^{141}$ S. Ali, ${ }^{157}$ M. Aliev,${ }^{165}$ G. Alimonti, ${ }^{69 a}$ C. Allaire, ${ }^{36}$ B. M. M. Allbrooke, ${ }^{155}$ B. W. Allen, ${ }^{131}$ P. P. Allport, ${ }^{21}$ A. Aloisio, ${ }^{70 a}{ }^{70 b}$ F. Alonso, ${ }^{89}$ C. Alpigiani, ${ }^{147}$ A. A. Alshehri, ${ }^{57}$ E. Alunno Camelia, ${ }^{74 a, 74 b}$ M. Alvarez Estevez,${ }^{99}$ M. G. Alviggi, ${ }^{70 a, 70 b}$ Y. Amaral Coutinho, ${ }^{81 b}$ A. Ambler,${ }^{104}$ L. Ambroz, ${ }^{134}$ C. Amelung, ${ }^{26}$ D. Amidei, ${ }^{106}$ S. P. Amor Dos Santos, ${ }^{139 a}$ S. Amoroso, ${ }^{46}$ C. S. Amrouche,${ }^{54}$ F. An, ${ }^{79}$ C. Anastopoulos, ${ }^{148}$ 
N. Andari, ${ }^{144}$ T. Andeen, ${ }^{11}$ C. F. Anders, ${ }^{61 b}$ J. K. Anders, ${ }^{20}$ S. Y. Andrean, ${ }^{45 a, 45 b}$ A. Andreazza,${ }^{69 a, 69 b}$ V. Andrei, ${ }^{61 \mathrm{a}}$ C. R. Anelli, ${ }^{175}$ S. Angelidakis, ${ }^{9}$ A. Angerami, ${ }^{39}$ A. V. Anisenkov, ${ }^{122 b, 122 a}$ A. Annovi, ${ }^{72 a}$ C. Antel,${ }^{54}$ M. T. Anthony,${ }^{148}$ E. Antipov, ${ }^{129}$ M. Antonelli, ${ }^{51}$ D. J. A. Antrim, ${ }^{170}$ F. Anulli, ${ }^{73 a}$ M. Aoki, ${ }^{82}$ J. A. Aparisi Pozo, ${ }^{173}$ M. A. Aparo, ${ }^{155}$ L. Aperio Bella, ${ }^{15 \mathrm{a}}$ V. Araujo Ferraz, ${ }^{81 \mathrm{~b}}$ R. Araujo Pereira,${ }^{81 \mathrm{~b}}$ C. Arcangeletti, ${ }^{51}$ A. T. H. Arce, ${ }^{49}$ F. A. Arduh ${ }^{89}$ J-F. Arguin, ${ }^{110}$ S. Argyropoulos, ${ }^{52}$ J.-H. Arling, ${ }^{46}$ A. J. Armbruster, ${ }^{36}$ A. Armstrong, ${ }^{170}$ O. Arnaez, ${ }^{166}$ H. Arnold, ${ }^{120}$ Z. P. Arrubarrena Tame, ${ }^{114}$ G. Artoni,${ }^{134}$ S. Artz, ${ }^{100}$ S. Asai, ${ }^{162}$ T. Asawatavonvanich, ${ }^{164}$ N. Asbah, ${ }^{59}$

E. M. Asimakopoulou, ${ }^{171}$ L. Asquith, ${ }^{155}$ J. Assahsah, ${ }^{35 d}$ K. Assamagan, ${ }^{29}$ R. Astalos, ${ }^{28 a}$ R. J. Atkin, ${ }^{33 a}$ M. Atkinson, ${ }^{172}$ N. B. Atlay, ${ }^{19}$ H. Atmani, ${ }^{65}$ K. Augsten, ${ }^{141}$ G. Avolio, ${ }^{36}$ M. K. Ayoub,${ }^{15 a}$ G. Azuelos, ${ }^{110, d}$ H. Bachacou, ${ }^{144}$ K. Bachas, ${ }^{161}$ M. Backes, ${ }^{134}$ F. Backman, ${ }^{45 a, 45 b}$ P. Bagnaia, ${ }^{73 a, 73 b}$ M. Bahmani, ${ }^{85}$ H. Bahrasemani, ${ }^{151}$ A. J. Bailey, ${ }^{173}$ V. R. Bailey, ${ }^{172}$ J. T. Baines, ${ }^{143}$ C. Bakalis, ${ }^{10}$ O. K. Baker, ${ }^{182}$ P. J. Bakker, ${ }^{120}$ D. Bakshi Gupta,${ }^{8}$ S. Balaji ${ }^{156}$ E. M. Baldin, ${ }^{122 b, 122 a}$ P. Balek, ${ }^{179}$ F. Balli, ${ }^{144}$ W. K. Balunas, ${ }^{134}$ J. Balz, ${ }^{100}$ E. Banas, ${ }^{85}$ M. Bandieramonte, ${ }^{138}$ A. Bandyopadhyay, ${ }^{24}$ Sw. Banerjee, ${ }^{180, e}$

L. Barak,${ }^{160}$ W. M. Barbe ${ }^{38}$ E. L. Barberio, ${ }^{105}$ D. Barberis,${ }^{55,55 a}$ M. Barbero, ${ }^{102}$ G. Barbour, ${ }^{95}$ T. Barillari, ${ }^{115}$ M-S. Barisits ${ }^{36}$ J. Barkeloo, ${ }^{131}$ T. Barklow, ${ }^{152}$ R. Barnea, ${ }^{159}$ B. M. Barnett, ${ }^{143}$ R. M. Barnett, ${ }^{18}$ Z. Barnovska-Blenessy, ${ }^{60 a}$ A. Baroncelli, ${ }^{60 a}$ G. Barone, ${ }^{29}$ A. J. Barr, ${ }^{134}$ L. Barranco Navarro, ${ }^{45 a, 45 b}$ F. Barreiro, ${ }^{99}$ J. Barreiro Guimarães da Costa, ${ }^{15 a}$ U. Barron, ${ }^{160}$ S. Barsov, ${ }^{137}$ F. Bartels,${ }^{61 a}$ R. Bartoldus, ${ }^{152}$ G. Bartolini, ${ }^{102}$ A. E. Barton,${ }^{90}$ P. Bartos, ${ }^{28 a}$ A. Basalaev, ${ }^{46}$ A. Basan, ${ }^{100}$ A. Bassalat, ${ }^{65, f}$ M. J. Basso, ${ }^{166}$ R. L. Bates,${ }^{57}$ S. Batlamous, ${ }^{35 e}$ J. R. Batley, ${ }^{32}$ B. Batool, ${ }^{150}$ M. Battaglia, ${ }^{145}$ M. Bauce, ${ }^{73 a, 73 b}$ F. Bauer, ${ }^{144}$ K. T. Bauer, ${ }^{170}$ H. S. Bawa,${ }^{31}$ J. B. Beacham, ${ }^{49}$ T. Beau, ${ }^{135}$ P. H. Beauchemin, ${ }^{169}$ F. Becherer,${ }^{52}$ P. Bechtle, ${ }^{24}$ H. C. Beck,${ }^{53}$ H. P. Beck, ${ }^{20, g}$ K. Becker, ${ }^{177}$ C. Becot, ${ }^{46}$ A. Beddall, ${ }^{12 \mathrm{~d}}$ A. J. Beddall, ${ }^{12 \mathrm{a}}$ V. A. Bednyakov,${ }^{80}$ M. Bedognetti, ${ }^{120}$ C. P. Bee, ${ }^{154}$ T. A. Beermann, ${ }^{181}$ M. Begalli, ${ }^{81 b}$ M. Begel,${ }^{29}$ A. Behera, ${ }^{154}$ J. K. Behr, ${ }^{46}$ F. Beisiegel, ${ }^{24}$ M. Belfkir, ${ }^{5}$ A. S. Bell, ${ }^{95}$ G. Bella, ${ }^{160}$ L. Bellagamba, ${ }^{23 b}$ A. Bellerive, ${ }^{34}$ P. Bellos, ${ }^{9}$ K. Beloborodov, ${ }^{122 b, 122 a}$ K. Belotskiy, ${ }^{112}$ N. L. Belyaev, ${ }^{112}$ D. Benchekroun, ${ }^{35 a}$ N. Benekos, ${ }^{10}$ Y. Benhammou, ${ }^{160}$ D. P. Benjamin, ${ }^{6}$ M. Benoit, ${ }^{54}$ J. R. Bensinger, ${ }^{26}$ S. Bentvelsen, ${ }^{120}$ L. Beresford, ${ }^{134}$ M. Beretta, ${ }^{51}$ D. Berge,${ }^{19}$ E. Bergeaas Kuutmann, ${ }^{171}$ N. Berger, ${ }^{5}$ B. Bergmann, ${ }^{141}$ L. J. Bergsten, ${ }^{26}$ J. Beringer, ${ }^{18}$ S. Berlendis, ${ }^{7}$ G. Bernardi, ${ }^{135}$ C. Bernius, ${ }^{152}$ F. U. Bernlochner, ${ }^{24}$ T. Berry, ${ }^{94}$ P. Berta, ${ }^{100}$ C. Bertella, ${ }^{15 a}$ A. Berthold, ${ }^{48}$ I. A. Bertram, ${ }^{90}$ O. Bessidskaia Bylund, ${ }^{181}$ N. Besson, ${ }^{144}$ A. Bethani, ${ }^{101}$ S. Bethke, ${ }^{115}$ A. Betti, ${ }^{42}$ A. J. Bevan, ${ }^{93}$ J. Beyer, ${ }^{115}$ D. S. Bhattacharya, ${ }^{176}$ P. Bhattarai, ${ }^{26}$ R. Bi, ${ }^{138}$ R. M. Bianchi, ${ }^{138}$ O. Biebel, ${ }^{114}$ D. Biedermann, ${ }^{19}$ R. Bielski, ${ }^{36}$ K. Bierwagen, ${ }^{100}$ N. V. Biesuz, ${ }^{72 a, 72 b}$ M. Biglietti, ${ }^{75 a}$ T. R. V. Billoud, ${ }^{110}$ M. Bindi, ${ }^{53}$ A. Bingul, ${ }^{12 \mathrm{~d}}$ C. Bini,${ }^{73 a, 73 b}$ S. Biondi, ${ }^{23 b, 23 a}$ M. Birman, ${ }^{179}$ T. Bisanz, ${ }^{53}$ J. P. Biswal, ${ }^{3}$ D. Biswas,${ }^{180, e}$ A. Bitadze, ${ }^{101}$ C. Bittrich ${ }^{48}$ K. Bjørke, ${ }^{133}$ T. Blazek, ${ }^{28 a}$ I. Bloch,${ }^{46}$ C. Blocker ${ }^{26}$ A. Blue,${ }^{57}$ U. Blumenschein, ${ }^{93}$ G. J. Bobbink, ${ }^{120}$ V. S. Bobrovnikov, ${ }^{122 b, 122 \mathrm{a}}$ S. S. Bocchetta, ${ }^{97}$ A. Bocci, ${ }^{49}$ D. Boerner, ${ }^{46}$ D. Bogavac, ${ }^{14}$ A. G. Bogdanchikov, ${ }^{122 b, 122 a}$ C. Bohm, ${ }^{45 a}$ V. Boisvert, ${ }^{94}$ P. Bokan,,${ }^{53,171}$ T. Bold, ${ }^{84 a}$ A. E. Bolz,${ }^{61 b}$ M. Bomben, ${ }^{135}$ M. Bona, ${ }^{93}$ J. S. Bonilla, ${ }^{131}$ M. Boonekamp, ${ }^{144}$ C. D. Booth, ${ }^{94}$ H. M. Borecka-Bielska, ${ }^{91}$ L. S. Borgna,${ }^{95}$ A. Borisov, ${ }^{123}$ G. Borissov, ${ }^{90}$ J. Bortfeldt, ${ }^{36}$ D. Bortoletto, ${ }^{134}$ D. Boscherini, ${ }^{23 \mathrm{~b}}$ M. Bosman, ${ }^{14}$ J. D. Bossio Sola, ${ }^{104}$ K. Bouaouda, ${ }^{35 a}$ J. Boudreau, ${ }^{138}$

E. V. Bouhova-Thacker, ${ }^{90}$ D. Boumediene, ${ }^{38}$ S. K. Boutle, ${ }^{57}$ A. Boveia, ${ }^{127}$ J. Boyd ${ }^{36}$ D. Boye,${ }^{33 c}$ I. R. Boyko, ${ }^{80}$ A. J. Bozson, ${ }^{94}$ J. Bracinik, ${ }^{21}$ N. Brahimi, ${ }^{102}$ G. Brandt, ${ }^{181}$ O. Brandt, ${ }^{32}$ F. Braren, ${ }^{46}$ B. Brau, ${ }^{103}$ J. E. Brau, ${ }^{131}$ W. D. Breaden Madden, ${ }^{57}$ K. Brendlinger ${ }^{46}$ L. Brenner, ${ }^{46}$ R. Brenner, ${ }^{171}$ S. Bressler, ${ }^{179}$ B. Brickwedde, ${ }^{100}$ D. L. Briglin, ${ }^{21}$ D. Britton, ${ }^{57}$ D. Britzger, ${ }^{115}$ I. Brock,${ }^{24}$ R. Brock,${ }^{107}$ G. Brooijmans, ${ }^{39}$ W. K. Brooks, ${ }^{146 \mathrm{~d}}$ E. Brost,${ }^{29}$

P. A. Bruckman de Renstrom, ${ }^{85}$ D. Bruncko, ${ }^{28 b}$ A. Bruni, ${ }^{23 b}$ G. Bruni, ${ }^{23 b}$ L. S. Bruni, ${ }^{120}$ S. Bruno, ${ }^{74 a, 74 b}$ M. Bruschi, ${ }^{23 b}$ N. Bruscino, ${ }^{73 a, 73 b}$ L. Bryngemark, ${ }^{97}$ T. Buanes, ${ }^{17}$ Q. Buat,${ }^{36}$ P. Buchholz, ${ }^{150}$ A. G. Buckley, ${ }^{57}$ I. A. Budagov, ${ }^{80}$ M. K. Bugge, ${ }^{133}$ F. Bührer, ${ }^{52}$ O. Bulekov, ${ }^{112}$ B. A. Bullard,${ }^{59}$ T. J. Burch, ${ }^{121}$ S. Burdin, ${ }^{91}$ C. D. Burgard, ${ }^{120}$ A. M. Burger, ${ }^{129}$ B. Burghgrave, ${ }^{8}$ J. T. P. Burr, ${ }^{46}$ C. D. Burton, ${ }^{11}$ J. C. Burzynski, ${ }^{103}$ V. Büscher, ${ }^{100}$ E. Buschmann, ${ }^{53}$ P. J. Bussey, ${ }^{57}$ J. M. Butler, ${ }^{25}$ C. M. Buttar, ${ }^{57}$ J. M. Butterworth, ${ }^{95}$ P. Butti ${ }^{36}$ W. Buttinger, ${ }^{36}$ C. J. Buxo Vazquez, ${ }^{107}$ A. Buzatu, ${ }^{157}$ A. R. Buzykaev, ${ }^{122 b, 122 a}$ G. Cabras, ${ }^{23 b, 23 a}$ S. Cabrera Urbán, ${ }^{173}$ D. Caforio,${ }^{56}$ H. Cai, ${ }^{172}$ V. M. M. Cairo, ${ }^{152}$ O. Cakir, ${ }^{4 a}$ N. Calace, ${ }^{36}$ P. Calafiura, ${ }^{18}$ G. Calderini, ${ }^{135}$ P. Calfayan, ${ }^{66}$ G. Callea, ${ }^{57}$ L. P. Caloba, ${ }^{81 b}$ A. Caltabiano,${ }^{74 a, 74 b}$ S. Calvente Lopez, ${ }^{99}$ D. Calvet, ${ }^{38}$ S. Calvet, ${ }^{38}$ T. P. Calvet, ${ }^{154}$ M. Calvetti, ${ }^{72 a, 72 b}$ R. Camacho Toro, ${ }^{135}$ S. Camarda ${ }^{36}$ D. Camarero Munoz, ${ }^{99}$ P. Camarri, ${ }^{74 a, 74 b}$ M. T. Camerlingo, ${ }^{75 a, 75 b}$ D. Cameron, ${ }^{133}$ C. Camincher, ${ }^{36}$ S. Campana, ${ }^{36}$ M. Campanelli, ${ }^{95}$ A. Camplani, ${ }^{40}$ A. Campoverde ${ }^{150}$ V. Canale,${ }^{70 a, 70 b}$ A. Canesse, ${ }^{104}$ M. Cano Bret, ${ }^{78}$ J. Cantero, ${ }^{129}$ T. Cao, ${ }^{160}$ Y. Cao, ${ }^{172}$ M. D. M. Capeans Garrido, ${ }^{36}$ M. Capua, ${ }^{41 \mathrm{~b}, 41 \mathrm{a}}$ R. Cardarelli, ${ }^{74 a}$ F. Cardillo, ${ }^{148}$ G. Carducci, ${ }^{41 b, 41 \mathrm{a}}$ I. Carli, ${ }^{142}$ T. Carli, ${ }^{36}$ G. Carlino, ${ }^{70 a}$ B. T. Carlson, ${ }^{138}$ E. M. Carlson, ${ }^{175,167 a}$ L. Carminati, ${ }^{69 a, 69 b}$ R. M. D. Carney, ${ }^{152}$ S. Caron, ${ }^{119}$ E. Carquin, ${ }^{146 \mathrm{~d}}$ S. Carrá, ${ }^{46}$ J. W. S. Carter, ${ }^{166}$ T. M. Carter, ${ }^{50}$ M. P. Casado, ${ }^{14, h}$ A. F. Casha, ${ }^{166}$ F. L. Castillo, ${ }^{173}$ 
L. Castillo Garcia, ${ }^{14}$ V. Castillo Gimenez, ${ }^{173}$ N. F. Castro, ${ }^{139 a, 139 e}$ A. Catinaccio, ${ }^{36}$ J. R. Catmore, ${ }^{133}$ A. Cattai ${ }^{36}$ V. Cavaliere, ${ }^{29}$ E. Cavallaro, ${ }^{14}$ V. Cavasinni, ${ }^{72 a, 72 b}$ E. Celebi,${ }^{12 b}$ F. Celli, ${ }^{134}$ L. Cerda Alberich, ${ }^{173}$ K. Cerny, ${ }^{130}$ A. S. Cerqueira, ${ }^{81 \mathrm{a}}$ A. Cerri, ${ }^{155}$ L. Cerrito, ${ }^{74 a, 74 b}$ F. Cerutti, ${ }^{18}$ A. Cervelli, ${ }^{23 b, 23 a}$ S. A. Cetin, ${ }^{12 b}$ Z. Chadi, ${ }^{35 a}$ D. Chakraborty, ${ }^{121}$ J. Chan, ${ }^{180}$ W. S. Chan, ${ }^{120}$ W. Y. Chan, ${ }^{91}$ J. D. Chapman, ${ }^{32}$ B. Chargeishvili, ${ }^{158 b}$ D. G. Charlton, ${ }^{21}$ T. P. Charman, ${ }^{93}$ C. C. Chau, ${ }^{34}$ S. Che, ${ }^{127}$ S. Chekanov, ${ }^{6}$ S. V. Chekulaev, ${ }^{167 a}$ G. A. Chelkov,${ }^{80, i}$ B. Chen,${ }^{79}$ C. Chen, ${ }^{60 a}$ C. H. Chen, ${ }^{79}$ H. Chen, ${ }^{29}$ J. Chen, ${ }^{60 \mathrm{a}}$ J. Chen, ${ }^{39}$ J. Chen, ${ }^{26}$ S. Chen, ${ }^{136}$ S. J. Chen, ${ }^{15 \mathrm{c}}$ X. Chen, ${ }^{15 \mathrm{~b}}$ Y. Chen, ${ }^{60 \mathrm{a}}$ Y-H. Chen, ${ }^{46}$ H. C. Cheng, ${ }^{63 \mathrm{a}}$ H. J. Cheng, ${ }^{15 a}$ A. Cheplakov, ${ }^{80}$ E. Cheremushkina, ${ }^{123}$ R. Cherkaoui El Moursli, ${ }^{35 \mathrm{e}}$ E. Cheu, ${ }^{7}$ K. Cheung, ${ }^{64}$ T. J. A. Chevalérias, ${ }^{144}$ L. Chevalier, ${ }^{144}$ V. Chiarella, ${ }^{51}$ G. Chiarelli, ${ }^{72 a}$ G. Chiodini, ${ }^{68 a}$ A. S. Chisholm, ${ }^{21}$ A. Chitan, ${ }^{27 b}$ I. Chiu, ${ }^{162}$ Y. H. Chiu, ${ }^{175}$ M. V. Chizhov, ${ }^{80}$ K. Choi, ${ }^{11}$ A. R. Chomont, ${ }^{73 a}, 73 b$ S. Chouridou, ${ }^{161}$ Y. S. Chow, ${ }^{120}$ L. D. Christopher, ${ }^{33 e}$ M. C. Chu, ${ }^{63 a}$ X. Chu, ${ }^{15 a, 15 d}$ J. Chudoba, ${ }^{140}$ J. J. Chwastowski, ${ }^{85}$ L. Chytka, ${ }^{130}$ D. Cieri, ${ }^{115}$ K. M. Ciesla,${ }^{85}$ D. Cinca, ${ }^{47}$ V. Cindro, ${ }^{92}$ I. A. Cioară, ${ }^{27 b}$ A. Ciocio, ${ }^{18}$ F. Cirotto, ${ }^{70 a, 70 b}$ Z. H. Citron, ${ }^{179, j}$ M. Citterio, ${ }^{69 a}$ D. A. Ciubotaru, ${ }^{27 b}$ B. M. Ciungu, ${ }^{166}$ A. Clark,${ }^{54}$ M. R. Clark, ${ }^{39}$ P. J. Clark,${ }^{50}$ S. E. Clawson, ${ }^{101}$ C. Clement, ${ }^{45 a, 45 b}$ Y. Coadou, ${ }^{102}$ M. Cobal, ${ }^{67 a, 67 c}$ A. Coccaro, ${ }^{55 b}$ J. Cochran, ${ }^{79}$ R. Coelho Lopes De Sa, ${ }^{103}$ H. Cohen, ${ }^{160}$ A. E. C. Coimbra, ${ }^{36}$ B. Cole, ${ }^{39}$ A. P. Colijn, ${ }^{120}$ J. Collot,${ }^{58}$ P. Conde Muiño, ${ }^{139 a, 139 h}$ S. H. Connell, ${ }^{33 \mathrm{c}}$ I. A. Connelly, ${ }^{57}$ S. Constantinescu, ${ }^{27 \mathrm{~b}}$ F. Conventi, ${ }^{70 a, k}$ A. M. Cooper-Sarkar, ${ }^{134}$ F. Cormier, ${ }^{174}$ K. J. R. Cormier, ${ }^{166}$ L. D. Corpe, ${ }^{95}$ M. Corradi, ${ }^{73 a, 73 b}$ E. E. Corrigan, ${ }^{97}$ F. Corriveau, ${ }^{104,1}$ M. J. Costa, ${ }^{173}$ F. Costanza, ${ }^{5}$ D. Costanzo,${ }^{148}$ G. Cowan, ${ }^{94}$ J. W. Cowley ${ }^{32}$ J. Crane,${ }^{101}$ K. Cranmer, ${ }^{125}$ S. J. Crawley, ${ }^{57}$ R. A. Creager, ${ }^{136}$ S. Crépé-Renaudin, ${ }^{58}$ F. Crescioli, ${ }^{135}$ M. Cristinziani, ${ }^{24}$ V. Croft, ${ }^{169}$ G. Crosetti, ${ }^{41 b, 41 a}$ A. Cueto, ${ }^{5}$ T. Cuhadar Donszelmann, ${ }^{170}$ A. R. Cukierman, ${ }^{152}$ W. R. Cunningham, ${ }^{57}$ S. Czekierda, ${ }^{85}$ P. Czodrowski, ${ }^{36}$ M. M. Czurylo, ${ }^{61 b}$ M. J. Da Cunha Sargedas De Sousa, ${ }^{60 b}$ J. V. Da Fonseca Pinto, ${ }^{81 b}$ C. Da Via, ${ }^{101}$ W. Dabrowski, ${ }^{84 a}$ F. Dachs,${ }^{36}$ T. Dado, ${ }^{28 a}$ S. Dahbi, ${ }^{33 e}$ T. Dai, ${ }^{106}$ C. Dallapiccola, ${ }^{103}$ M. Dam, ${ }^{40}$ G. D'amen,${ }^{29}$ V. D'Amico, ${ }^{75 a, 75 b}$ J. Damp, ${ }^{100}$ J. R. Dandoy, ${ }^{136}$ M. F. Daneri, ${ }^{30}$ N. S. Dann, ${ }^{101}$ M. Danninger, ${ }^{151}$ V. Dao, ${ }^{36}$ G. Darbo, ${ }^{55 b}$ O. Dartsi, ${ }^{5}$ A. Dattagupta, ${ }^{131}$ T. Daubney, ${ }^{46}$ S. D'Auria, ${ }^{69 a 69 b}$ C. David, ${ }^{167 b}$ T. Davidek, ${ }^{142}$ D. R. Davis, ${ }^{49}$ I. Dawson, ${ }^{148}$ K. De,${ }^{8}$ R. De Asmundis, ${ }^{70 a}$ M. De Beurs, ${ }^{120}$ S. De Castro, ${ }^{23 b, 23 a}$ S. De Cecco,${ }^{73 a, 73 b}$ N. De Groot, ${ }^{119}$ P. de Jong, ${ }^{120}$ H. De la Torre, ${ }^{107}$ A. De Maria, ${ }^{15 c}$ D. De Pedis, ${ }^{73 a}$ A. De Salvo,${ }^{73 a}$ U. De Sanctis, ${ }^{74 a, 74 b}$ M. De Santis, ${ }^{74 a, 74 b}$ A. De Santo, ${ }^{155}$ K. De Vasconcelos Corga,${ }^{102}$ J. B. De Vivie De Regie ${ }^{65}$ C. Debenedetti, ${ }^{145}$ D. V. Dedovich, ${ }^{80}$ A. M. Deiana,${ }^{42}$ J. Del Peso, ${ }^{99}$ Y. Delabat Diaz ${ }^{46}$ D. Delgove ${ }^{65}$ F. Deliot,${ }^{144, \mathrm{~m}}$ C. M. Delitzsch, ${ }^{7}$ M. Della Pietra,${ }^{70 a}{ }^{70 b}$ D. Della Volpe,${ }^{54}$ A. Dell' Acqua,${ }^{36}$ L. Dell'Asta, ${ }^{74 a, 74 b}$ M. Delmastro, ${ }^{5}$ C. Delporte ${ }^{65}$ P. A. Delsart ${ }^{58}$ D. A. DeMarco, ${ }^{166}$ S. Demers ${ }^{182}$ M. Demichev, ${ }^{80}$ G. Demontigny, ${ }^{110}$ S. P. Denisov, ${ }^{123}$ L. D'Eramo, ${ }^{121}$ D. Derendarz,${ }^{85}$ J. E. Derkaoui, ${ }^{35 d}$ F. Derue, ${ }^{135}$ P. Dervan, ${ }^{91}$ K. Desch,${ }^{24}$ C. Deterre ${ }^{46}$ K. Dette, ${ }^{166}$ C. Deutsch, ${ }^{24}$ M. R. Devesa,${ }^{30}$ P. O. Deviveiros,${ }^{36}$ F. A. Di Bello, ${ }^{73 a, 73 b}$ A. Di Ciaccio, ${ }^{74 a, 74 b}$ L. Di Ciaccio, ${ }^{5}$ W. K. Di Clemente, ${ }^{136}$ C. Di Donato ${ }^{70 a, 70 b}$ A. Di Girolamo, ${ }^{36}$ G. Di Gregorio, ${ }^{72 a, 72 b}$ B. Di Micco, ${ }^{75 a, 75 b}$ R. Di Nardo, ${ }^{75 a, 75 b}$ K. F. Di Petrillo, ${ }^{59}$ R. Di Sipio, ${ }^{166}$ C. Diaconu, ${ }^{102}$ F. A. Dias, ${ }^{40}$ T. Dias Do Vale, ${ }^{139 a}$ M. A. Diaz, ${ }^{146 a}$ F. G. Diaz Capriles ${ }^{24}$ J. Dickinson, ${ }^{18}$ E. B. Diehl,${ }^{106}$ J. Dietrich, ${ }^{19}$ S. Díez Cornell,${ }^{46}$ A. Dimitrievska,${ }^{18}$ W. Ding ${ }^{15 b}$

J. Dingfelder ${ }^{24}$ F. Dittus,${ }^{36}$ F. Djama, ${ }^{102}$ T. Djobava, ${ }^{158 b}$ J. I. Djuvsland, ${ }^{17}$ M. A. B. Do Vale,${ }^{81 \mathrm{c}}$ M. Dobre,${ }^{27 \mathrm{~b}}$

D. Dodsworth, ${ }^{26}$ C. Doglioni,${ }^{97}$ J. Dolejsi,${ }^{142}$ Z. Dolezal, ${ }^{142}$ M. Donadelli, ${ }^{81 \mathrm{~d}}$ B. Dong,${ }^{60 \mathrm{c}}$ J. Donini,${ }^{38}$ A. D'onofrio, ${ }^{15 \mathrm{c}}$ M. D’Onofrio, ${ }^{91}$ J. Dopke, ${ }^{143}$ A. Doria,${ }^{70 a}$ M. T. Dova, ${ }^{89}$ A. T. Doyle, ${ }^{57}$ E. Drechsler, ${ }^{151}$ E. Dreyer, ${ }^{151}$ T. Dreyer, ${ }^{53}$ A. S. Drobac, ${ }^{169}$ D. Du, ${ }^{60 b}$ T. A. du Pree, ${ }^{120}$ Y. Duan, ${ }^{60 b}$ F. Dubinin, ${ }^{111}$ M. Dubovsky, ${ }^{28 a}$ A. Dubreuil, ${ }^{54}$ E. Duchovni, ${ }^{179}$ G. Duckeck, ${ }^{114}$ O. A. Ducu, ${ }^{110}$ D. Duda, ${ }^{115}$ A. Dudarev, ${ }^{36}$ A. C. Dudder, ${ }^{100}$ E. M. Duffield, ${ }^{18}$ L. Duflot, ${ }^{65}$ M. Dührssen, ${ }^{36}$ C. Dülsen, ${ }^{181}$ M. Dumancic, ${ }^{179}$ A. E. Dumitriu, ${ }^{27 b}$ A. K. Duncan, ${ }^{57}$ M. Dunford, ${ }^{61 a}$ A. Duperrin, ${ }^{102}$ H. Duran Yildiz,${ }^{4 a}$ M. Düren, ${ }^{56}$ A. Durglishvili, ${ }^{158 b}$ D. Duschinger, ${ }^{48}$ B. Dutta, ${ }^{46}$ D. Duvnjak, ${ }^{1}$ G. I. Dyckes,${ }^{136}$ M. Dyndal, ${ }^{36}$ S. Dysch,${ }^{101}$ B. S. Dziedzic, ${ }^{85}$ K. M. Ecker, ${ }^{115}$ M. G. Eggleston, ${ }^{49}$ T. Eifert, ${ }^{8}$ G. Eigen, ${ }^{17}$ K. Einsweiler,${ }^{18}$ T. Ekelof, ${ }^{171}$ H. El Jarrari, ${ }^{35 e}$ R. El Kosseifi, ${ }^{102}$ V. Ellajosyula, ${ }^{171}$ M. Ellert, ${ }^{171}$ F. Ellinghaus, ${ }^{181}$ A. A. Elliot, ${ }^{93}$ N. Ellis, ${ }^{36}$ J. Elmsheuser, ${ }^{29}$ M. Elsing, ${ }^{36}$ D. Emeliyanov, ${ }^{143}$ A. Emerman, ${ }^{39}$ Y. Enari, ${ }^{162}$ M. B. Epland, ${ }^{49}$ J. Erdmann, ${ }^{47}$ A. Ereditato, ${ }^{20}$ P. A. Erland,${ }^{85}$ M. Errenst,${ }^{36}$ M. Escalier ${ }^{65}$ C. Escobar, ${ }^{173}$ O. Estrada Pastor, ${ }^{173}$ E. Etzion, ${ }^{160}$ H. Evans, ${ }^{66}$ M. O. Evans, ${ }^{155}$ A. Ezhilov, ${ }^{137}$ F. Fabbri ${ }^{57}$ L. Fabbri, ${ }^{23 b, 23 a}$ V. Fabiani, ${ }^{119}$ G. Facini, ${ }^{177}$ R. M. Faisca Rodrigues Pereira ${ }^{139 a}$ R. M. Fakhrutdinov, ${ }^{123}$ S. Falciano, ${ }^{73 a}$ P. J. Falke, ${ }^{24}$ S. Falke, ${ }^{36}$ J. Faltova, ${ }^{142}$ Y. Fang, ${ }^{15 a}$ Y. Fang, ${ }^{15 a}$ G. Fanourakis,${ }^{44}$ M. Fanti, ${ }^{69 a, 69 b}$ M. Faraj, ${ }^{67 a, 67 c, n}$ A. Farbin, ${ }^{8}$ A. Farilla, ${ }^{75 a}$ E. M. Farina, ${ }^{71 a, 71 b}$ T. Farooque, ${ }^{107}$ S. M. Farrington, ${ }^{50}$ P. Farthouat, ${ }^{36}$ F. Fassi, ${ }^{35 \mathrm{e}}$ P. Fassnacht, ${ }^{36}$

D. Fassouliotis, ${ }^{9}$ M. Faucci Giannelli, ${ }^{50}$ W. J. Fawcett, ${ }^{32}$ L. Fayard, ${ }^{65}$ O. L. Fedin, ${ }^{137, \mathrm{o}}$ W. Fedorko, ${ }^{174}$ A. Fehr, ${ }^{20}$ M. Feickert ${ }^{172}$ L. Feligioni, ${ }^{102}$ A. Fell, ${ }^{178}$ C. Feng, ${ }^{60 b}$ M. Feng, ${ }^{49}$ M. J. Fenton, ${ }^{170}$ A. B. Fenyuk, ${ }^{123}$ S. W. Ferguson, ${ }^{43}$ J. Ferrando, ${ }^{46}$ A. Ferrante, ${ }^{172}$ A. Ferrari, ${ }^{171}$ P. Ferrari, ${ }^{120}$ R. Ferrari, ${ }^{71 a}$ D. E. Ferreira de Lima, ${ }^{61 b}$ A. Ferrer, ${ }^{173}$ D. Ferrere, ${ }^{54}$ 
C. Ferretti, ${ }^{106}$ F. Fiedler, ${ }^{100}$ A. Filipčič,${ }^{92}$ F. Filthaut, ${ }^{119}$ K. D. Finelli, ${ }^{25}$ M. C. N. Fiolhais, ${ }^{139 a, 139 c, p}$ L. Fiorini, ${ }^{173}$ F. Fischer, ${ }^{114}$ W. C. Fisher, ${ }^{107}$ I. Fleck, ${ }^{150}$ P. Fleischmann, ${ }^{106}$ T. Flick, ${ }^{181}$ B. M. Flierl, ${ }^{114}$ L. Flores, ${ }^{136}$ L. R. Flores Castillo, ${ }^{63 a}$ F. M. Follega ${ }^{76 a, 76 b}$ N. Fomin, ${ }^{17}$ J. H. Foo, ${ }^{166}$ G. T. Forcolin, ${ }^{76 a, 76 b}$ A. Formica, ${ }^{144}$ F. A. Förster, ${ }^{14}$ A. C. Forti, ${ }^{101}$ E. Fortin, ${ }^{102}$ M. G. Foti, ${ }^{134}$ D. Fournier, ${ }^{65}$ H. Fox,${ }^{90}$ P. Francavilla, ${ }^{72 a, 72 b}$ S. Francescato, ${ }^{73 a, 73 b}$ M. Franchini, ${ }^{23 b, 23 a}$ S. Franchino, ${ }^{61 a}$ D. Francis, ${ }^{36}$ L. Franco, ${ }^{5}$ L. Franconi, ${ }^{20}$ M. Franklin, ${ }^{59}$ A. N. Fray, ${ }^{93}$ P. M. Freeman, ${ }^{21}$ B. Freund, ${ }^{110}$ W. S. Freund, ${ }^{81 b}$ E. M. Freundlich ${ }^{47}$ D. C. Frizzell, ${ }^{128}$ D. Froidevaux,${ }^{36}$ J. A. Frost,${ }^{134}$ M. Fujimoto, ${ }^{126}$ C. Fukunaga, ${ }^{163}$

E. Fullana Torregrosa, ${ }^{173}$ T. Fusayasu, ${ }^{116}$ J. Fuster, ${ }^{173}$ A. Gabrielli, ${ }^{23 b, 23 a}$ A. Gabrielli, ${ }^{18}$ S. Gadatsch, ${ }^{54}$ P. Gadow, ${ }^{115}$ G. Gagliardi, ${ }^{55 b, 55 a}$ L. G. Gagnon, ${ }^{110}$ B. Galhardo, ${ }^{139 a}$ G. E. Gallardo, ${ }^{134}$ E. J. Gallas, ${ }^{134}$ B. J. Gallop, ${ }^{143}$ G. Galster, ${ }^{40}$ R. Gamboa Goni, ${ }^{93}$ K. K. Gan, ${ }^{127}$ S. Ganguly, ${ }^{179}$ J. Gao, ${ }^{60 a}$ Y. Gao, ${ }^{50}$ Y. S. Gao, ${ }^{31, q}$ C. García, ${ }^{173}$ J. E. García Navarro, ${ }^{173}$ J. A. García Pascual, ${ }^{15 a}$ C. Garcia-Argos,${ }^{52}$ M. Garcia-Sciveres, ${ }^{18}$ R. W. Gardner, ${ }^{37}$ N. Garelli, ${ }^{152}$ S. Gargiulo, ${ }^{52}$ C. A. Garner, ${ }^{166}$ V. Garonne, ${ }^{133}$ S. J. Gasiorowski, ${ }^{147}$ P. Gaspar, ${ }^{81 b}$ A. Gaudiello, ${ }^{55 b, 55 a}$ G. Gaudio, ${ }^{71 a}$ I. L. Gavrilenko, ${ }^{111}$ A. Gavrilyuk, ${ }^{124}$ C. Gay, ${ }^{174}$ G. Gaycken,${ }^{46}$ E. N. Gazis,${ }^{10}$ A. A. Geanta, ${ }^{27 b}$ C. M. Gee,${ }^{145}$ C. N. P. Gee,${ }^{143}$ J. Geisen, ${ }^{97}$ M. Geisen, ${ }^{100}$ C. Gemme, ${ }^{55 b}$ M. H. Genest, ${ }^{58}$ C. Geng, ${ }^{106}$ S. Gentile, ${ }^{73 a, 73 b}$ S. George, ${ }^{94}$ T. Geralis, ${ }^{44}$ L. O. Gerlach, ${ }^{53}$ P. Gessinger-Befurt, ${ }^{100}$ G. Gessner, ${ }^{47}$ S. Ghasemi, ${ }^{150}$ M. Ghasemi Bostanabad, ${ }^{175}$ M. Ghneimat, ${ }^{150}$ A. Ghosh, ${ }^{65}$ A. Ghosh, ${ }^{78}$ B. Giacobbe, ${ }^{23 b}$ S. Giagu, ${ }^{73 a, 73 b}$ N. Giangiacomi, ${ }^{23 b, 23 a}$ P. Giannetti, ${ }^{72 a}$ A. Giannini, ${ }^{70 a, 70 b}$ G. Giannini, ${ }^{14}$ S. M. Gibson, ${ }^{94}$ M. Gignac, ${ }^{145}$ D. Gillberg, ${ }^{34}$ G. Gilles, ${ }^{181}$ D. M. Gingrich, ${ }^{3, d}$ M. P. Giordani, ${ }^{67 a, 67 c}$ P. F. Giraud, ${ }^{144}$ G. Giugliarelli, ${ }^{67 a, 67 c}$ D. Giugni, ${ }^{69 a}$ F. Giuli, ${ }^{74 a} 74 \mathrm{~b}$ S. Gkaitatzis, ${ }^{161}$ I. Gkialas, ${ }^{9, \mathrm{r}}$ E. L. Gkougkousis, ${ }^{14}$ P. Gkountoumis, ${ }^{10}$ L. K. Gladilin, ${ }^{113}$ C. Glasman, ${ }^{99}$ J. Glatzer, ${ }^{14}$ P. C. F. Glaysher, ${ }^{46}$ A. Glazov, ${ }^{46}$ G. R. Gledhill, ${ }^{131}$ I. Gnesi, ${ }^{41 b}$ M. Goblirsch-Kolb, ${ }^{26}$ D. Godin,,${ }^{110}$ S. Goldfarb, ${ }^{105}$ T. Golling, ${ }^{54}$ D. Golubkov, ${ }^{123}$ A. Gomes, ${ }^{139 a, 139 b}$ R. Goncalves Gama, ${ }^{53}$ R. Gonçalo, ${ }^{139 a}$ G. Gonella, ${ }^{131}$ L. Gonella, ${ }^{21}$ A. Gongadze, ${ }^{80}$ F. Gonnella, ${ }^{21}$ J. L. Gonski, ${ }^{39}$ S. González de la Hoz, ${ }^{173}$ S. Gonzalez Fernandez, ${ }^{14}$ C. Gonzalez Renteria, ${ }^{18}$ R. Gonzalez Suarez, ${ }^{171}$ S. Gonzalez-Sevilla, ${ }^{54}$ G. R. Gonzalvo Rodriguez, ${ }^{173}$ L. Goossens, ${ }^{36}$ N. A. Gorasia, ${ }^{21}$ P. A. Gorbounov, ${ }^{124}$ H. A. Gordon, ${ }^{29}$ B. Gorini, ${ }^{36}$ E. Gorini, ${ }^{68,68 b}$ A. Gorišek, ${ }^{92}$ A. T. Goshaw, ${ }^{49}$ M. I. Gostkin, ${ }^{80}$ C. A. Gottardo, ${ }^{119}$ M. Gouighri, ${ }^{35 b}$ A. G. Goussiou, ${ }^{147}$ N. Govender, ${ }^{33 c}$ C. Goy, ${ }^{5}$ E. Gozani, ${ }^{159}$ I. Grabowska-Bold, ${ }^{84 a}$ E. C. Graham, ${ }^{91}$ J. Gramling, ${ }^{170}$ E. Gramstad, ${ }^{133}$ S. Grancagnolo, ${ }^{19}$ M. Grandi, ${ }^{155}$ V. Gratchev, ${ }^{137}$ P. M. Gravila, ${ }^{27 f}$ F. G. Gravili, ${ }^{68 a, 68 b}$ C. Gray,${ }^{57}$ H. M. Gray, ${ }^{18}$ C. Grefe,${ }^{24}$ K. Gregersen, ${ }^{97}$ I. M. Gregor, ${ }^{46}$ P. Grenier, ${ }^{152}$ K. Grevtsov, ${ }^{46}$ C. Grieco, ${ }^{14}$ N. A. Grieser, ${ }^{128}$ A. A. Grillo, ${ }^{145}$ K. Grimm, ${ }^{31, s}$ S. Grinstein, ${ }^{14, t}$ J.-F. Grivaz, ${ }^{65}$ S. Groh, ${ }^{100}$ E. Gross, ${ }^{179}$ J. Grosse-Knetter, ${ }^{53}$ Z. J. Grout, ${ }^{95}$ C. Grud, ${ }^{106}$ A. Grummer, ${ }^{118}$ J. C. Grundy, ${ }^{134}$ L. Guan, ${ }^{106}$ W. Guan, ${ }^{180}$ C. Gubbels, ${ }^{174}$ J. Guenther, ${ }^{36}$ A. Guerguichon, ${ }^{65}$ J. G. R. Guerrero Rojas, ${ }^{173}$ F. Guescini, ${ }^{115}$ D. Guest, ${ }^{170}$ R. Gugel, ${ }^{52}$ T. Guillemin, ${ }^{5}$ S. Guindon, ${ }^{36}$ U. Gul,${ }^{57}$ J. Guo, ${ }^{60 \mathrm{c}}$ W. Guo, ${ }^{106}$ Y. Guo, ${ }^{60 \mathrm{a}}$ Z. Guo, ${ }^{102}$ R. Gupta, ${ }^{46}$ S. Gurbuz, ${ }^{12 \mathrm{c}}$ G. Gustavino, ${ }^{128}$ M. Guth, ${ }^{52}$ P. Gutierrez, ${ }^{128}$ C. Gutschow,${ }^{95}$ C. Guyot, ${ }^{144}$ C. Gwenlan, ${ }^{134}$ C. B. Gwilliam, ${ }^{91}$ A. Haas, ${ }^{125}$ C. Haber, ${ }^{18}$ H. K. Hadavand ${ }^{8}$ A. Hadef,${ }^{60 a}$ M. Haleem, ${ }^{176}$ J. Haley, ${ }^{129}$ J. J. Hall, ${ }^{148}$ G. Halladjian, ${ }^{107}$ G. D. Hallewell, ${ }^{102}$ K. Hamacher, ${ }^{181}$ P. Hamal, ${ }^{130}$ K. Hamano, ${ }^{175}$ H. Hamdaoui, ${ }^{350}$ M. Hamer, ${ }^{24}$ G. N. Hamity, ${ }^{50}$ K. Han,${ }^{60 a, u}$ L. Han, ${ }^{60 a}$ S. Han, ${ }^{15 a}$ Y. F. Han,${ }^{166}$ K. Hanagaki, ${ }^{82, v}$ M. Hance, ${ }^{145}$ D. M. Handl,${ }^{114}$ B. Haney, ${ }^{136}$ M. D. Hank, ${ }^{37}$ R. Hankache, ${ }^{135}$ E. Hansen, ${ }^{97}$ J. B. Hansen, ${ }^{40}$ J. D. Hansen, ${ }^{40}$ M. C. Hansen, ${ }^{24}$ P. H. Hansen, ${ }^{40}$ E. C. Hanson, ${ }^{101}$ K. Hara, ${ }^{168}$ T. Harenberg, ${ }^{181}$ S. Harkusha, ${ }^{108}$ P. F. Harrison, ${ }^{177}$ N. M. Hartman, ${ }^{152}$ N. M. Hartmann, ${ }^{114}$ Y. Hasegawa, ${ }^{149}$ A. Hasib, ${ }^{50}$ S. Hassani ${ }^{144}$

S. Haug ${ }^{20}$ R. Hauser,${ }^{107}$ L. B. Havener ${ }^{39}$ M. Havranek, ${ }^{141}$ C. M. Hawkes,${ }^{21}$ R. J. Hawkings, ${ }^{36}$ S. Hayashida, ${ }^{117}$

D. Hayden, ${ }^{107}$ C. Hayes,${ }^{106}$ R. L. Hayes, ${ }^{174}$ C. P. Hays,${ }^{134}$ J. M. Hays,${ }^{93}$ H. S. Hayward, ${ }^{91}$ S. J. Haywood, ${ }^{143}$ F. He, ${ }^{60 a}$ M. P. Heath ${ }^{50}$ V. Hedberg, ${ }^{97}$ S. Heer, ${ }^{24}$ A. L. Heggelund, ${ }^{133}$ K. K. Heidegger, ${ }^{52}$ W. D. Heidorn, ${ }^{79}$ J. Heilman, ${ }^{34}$ S. Heim,${ }^{46}$ T. Heim, ${ }^{18}$ B. Heinemann, ${ }^{46, w}$ J. J. Heinrich, ${ }^{131}$ L. Heinrich, ${ }^{36}$ J. Hejbal, ${ }^{140}$ L. Helary, ${ }^{61 b}$ A. Held, ${ }^{125}$ S. Hellesund, ${ }^{133}$ C. M. Helling, ${ }^{145}$ S. Hellman, ${ }^{45 a, 45 b}$ C. Helsens, ${ }^{36}$ R. C. W. Henderson, ${ }^{90}$ Y. Heng, ${ }^{180}$ L. Henkelmann, ${ }^{32}$

A. M. Henriques Correia, ${ }^{36}$ H. Herde,${ }^{26}$ Y. Hernández Jiménez, ${ }^{33 e}$ H. Herr, ${ }^{100}$ M. G. Herrmann, ${ }^{114}$ T. Herrmann, ${ }^{48}$ G. Herten,${ }^{52}$ R. Hertenberger, ${ }^{114}$ L. Hervas,${ }^{36}$ T. C. Herwig, ${ }^{136}$ G. G. Hesketh, ${ }^{95}$ N. P. Hessey,${ }^{167 a}$ H. Hibi, ${ }^{83}$ A. Higashida, ${ }^{162}$ S. Higashino, ${ }^{82}$ E. Higón-Rodriguez, ${ }^{173}$ K. Hildebrand, ${ }^{37}$ J. C. Hill, ${ }^{32}$ K. K. Hill,${ }^{29}$ K. H. Hiller, ${ }^{46}$ S. J. Hillier, ${ }^{21}$ M. Hils, ${ }^{48}$ I. Hinchliffe, ${ }^{18}$ F. Hinterkeuser, ${ }^{24}$ M. Hirose, ${ }^{132}$ S. Hirose, ${ }^{52}$ D. Hirschbuehl, ${ }^{181}$ B. Hiti, ${ }^{92}$ O. Hladik, ${ }^{140}$ D. R. Hlaluku, ${ }^{33 e}$ J. Hobbs, ${ }^{154}$ N. Hod, ${ }^{179}$ M. C. Hodgkinson, ${ }^{148}$ A. Hoecker, ${ }^{36}$ D. Hohn, ${ }^{52}$ D. Hohov, ${ }^{65}$ T. Holm, ${ }^{24}$ T. R. Holmes, ${ }^{37}$ M. Holzbock, ${ }^{114}$ L. B. A. H. Hommels, ${ }^{32}$ T. M. Hong, ${ }^{138}$ J. C. Honig, ${ }^{52}$ A. Hönle ${ }^{115}$ B. H. Hooberman, ${ }^{172}$ W. H. Hopkins, ${ }^{6}$ Y. Horii, ${ }^{117}$ P. Horn, ${ }^{48}$ L. A. Horyn, ${ }^{37}$ S. Hou,${ }^{157}$ A. Hoummada, ${ }^{35 a}$ J. Howarth,${ }^{57}$ J. Hoya, ${ }^{89}$ M. Hrabovsky, ${ }^{130}$ J. Hrdinka,${ }^{77}$ I. Hristova, ${ }^{19}$ J. Hrivnac, ${ }^{65}$ A. Hrynevich, ${ }^{109}$ T. Hryn'ova, ${ }^{5}$ P. J. Hsu, ${ }^{64}$ S.-C. Hsu, ${ }^{147}$ Q. Hu, ${ }^{29}$ S. Hu, ${ }^{60 \mathrm{c}}$ Y. F. Hu, ${ }^{15 a, 15 d}$ D. P. Huang, ${ }^{95}$ X. Huang, ${ }^{15 c}$ Y. Huang, ${ }^{60 a}$ Y. Huang, ${ }^{15 a}$ Z. Hubacek, ${ }^{141}$ F. Hubaut, ${ }^{102}$ M. Huebner, ${ }^{24}$ F. Huegging, ${ }^{24}$ 
T. B. Huffman, ${ }^{134}$ M. Huhtinen, ${ }^{36}$ R. F. H. Hunter ${ }^{34}$ P. Huo, ${ }^{154}$ N. Huseynov, ${ }^{80, x}$ J. Huston, ${ }^{107}$ J. Huth,${ }^{59}$ R. Hyneman, ${ }^{106}$ S. Hyrych, ${ }^{28 a}$ G. Iacobucci, ${ }^{54}$ G. Iakovidis, ${ }^{29}$ I. Ibragimov, ${ }^{150}$ L. Iconomidou-Fayard ${ }^{65}$ P. Iengo,${ }^{36}$ R. Ignazzi, ${ }^{40}$ O. Igonkina, ${ }^{120, a, y}$ R. Iguchi, ${ }^{162}$ T. Iizawa, ${ }^{54}$ Y. Ikegami, ${ }^{82}$ M. Ikeno, ${ }^{82}$ D. Iliadis, ${ }^{161}$ N. Ilic, ${ }^{119,166,1} \mathrm{~F}$. Iltzsche,${ }^{48}$ H. Imam, ${ }^{35 a}$ G. Introzzi, ${ }^{71 a, 71 b}$ M. Iodice, ${ }^{75 a}$ K. Iordanidou, ${ }^{167 a}$ V. Ippolito, ${ }^{73 a, 73 b}$ M. F. Isacson, ${ }^{171}$ M. Ishino, ${ }^{162}$ W. Islam, ${ }^{129}$

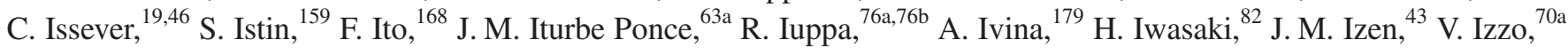
P. Jacka, ${ }^{140}$ P. Jackson, ${ }^{1}$ R. M. Jacobs,${ }^{46}$ B. P. Jaeger, ${ }^{151}$ V. Jain, ${ }^{2}$ G. Jäkel, ${ }^{181}$ K. B. Jakobi,${ }^{100}$ K. Jakobs,${ }^{52}$ T. Jakoubek, ${ }^{140}$ J. Jamieson, ${ }^{57}$ K. W. Janas, ${ }^{84 a}$ R. Jansky,${ }^{54}$ M. Janus, ${ }^{53}$ P. A. Janus, ${ }^{84 a}$ G. Jarlskog, ${ }^{97}$ A. E. Jaspan, ${ }^{91}$ N. Javadov, ${ }^{80, x}$

T. Javůrek, ${ }^{36}$ M. Javurkova, ${ }^{103}$ F. Jeanneau, ${ }^{144}$ L. Jeanty, ${ }^{131}$ J. Jejelava, ${ }^{158 a}$ A. Jelinskas, ${ }^{177}$ P. Jenni, ${ }^{52, z}$ N. Jeong, ${ }^{46}$ S. Jézéquel, ${ }^{5}$ H. Ji, ${ }^{180}$ J. Jia, ${ }^{154}$ H. Jiang, ${ }^{79}$ Y. Jiang, ${ }^{60 a}$ Z. Jiang, ${ }^{152}$ S. Jiggins, ${ }^{52}$ F. A. Jimenez Morales, ${ }^{38}$ J. Jimenez Pena, ${ }^{115}$ S. Jin, ${ }^{15 \mathrm{c}}$ A. Jinaru, ${ }^{27 b}$ O. Jinnouchi, ${ }^{164}$ H. Jivan, ${ }^{33 \mathrm{e}}$ P. Johansson, ${ }^{148}$ K. A. Johns, ${ }^{7}$ C. A. Johnson, ${ }^{66}$ R. W. L. Jones,${ }^{90}$ S. D. Jones, ${ }^{155}$ S. Jones, ${ }^{7}$ T. J. Jones, ${ }^{91}$ J. Jongmanns, ${ }^{61 a}$ P. M. Jorge, ${ }^{139 a}$ J. Jovicevic, ${ }^{36}$ X. Ju, ${ }^{18}$ J. J. Junggeburth, ${ }^{115}$ A. Juste Rozas, ${ }^{14, t}$ A. Kaczmarska ${ }^{85}$ M. Kado,${ }^{73 a, 73 b}$ H. Kagan, ${ }^{127}$ M. Kagan ${ }^{152}$ A. Kahn, ${ }^{39}$ C. Kahra, ${ }^{100}$ T. Kaji, ${ }^{178}$ E. Kajomovitz, ${ }^{159}$ C. W. Kalderon, ${ }^{29}$ A. Kaluza, ${ }^{100}$ A. Kamenshchikov, ${ }^{123}$ M. Kaneda, ${ }^{162}$ N. J. Kang, ${ }^{145}$ S. Kang, ${ }^{79}$ Y. Kano, ${ }^{117}$ J. Kanzaki, ${ }^{82}$ L. S. Kaplan, ${ }^{180}$ D. Kar,${ }^{33 e}$ K. Karava, ${ }^{134}$ M. J. Kareem, ${ }^{167 b}$ I. Karkanias, ${ }^{161}$ S. N. Karpov, ${ }^{80}$ Z. M. Karpova ${ }^{80}$ V. Kartvelishvili, ${ }^{90}$ A. N. Karyukhin, ${ }^{123}$ A. Kastanas, ${ }^{45 a, 45 b}$ C. Kato, ${ }^{60 d, 60 c}$ J. Katzy, ${ }^{46}$ K. Kawade, ${ }^{149}$ K. Kawagoe, ${ }^{88}$ T. Kawaguchi, ${ }^{117}$ T. Kawamoto, ${ }^{144}$ G. Kawamura, ${ }^{53}$ E. F. Kay, ${ }^{175}$ S. Kazakos, ${ }^{14}$ V. F. Kazanin, ${ }^{122 b, 122 a}$ R. Keeler ${ }^{175}$ R. Kehoe, ${ }^{42}$ J. S. Keller, ${ }^{34}$ E. Kellermann, ${ }^{97}$ D. Kelsey, ${ }^{155}$ J. J. Kempster, ${ }^{21}$ J. Kendrick, ${ }^{21}$ K. E. Kennedy ${ }^{39}$ O. Kepka, ${ }^{140}$ S. Kersten, ${ }^{181}$ B. P. Kerševan, ${ }^{92}$ S. Ketabchi Haghighat, ${ }^{166}$ M. Khader, ${ }^{172}$ F. Khalil-Zada, ${ }^{13}$ M. Khandoga, ${ }^{144}$ A. Khanov, ${ }^{129}$ A. G. Kharlamov, ${ }^{122 b, 122 a}$ T. Kharlamova, ${ }^{122 b, 122 a}$ E. E. Khoda, ${ }^{174}$ A. Khodinov, ${ }^{165}$ T. J. Khoo, ${ }^{54}$ G. Khoriauli, ${ }^{176}$ E. Khramov, ${ }^{80}$ J. Khubua, ${ }^{158 b}$ S. Kido ${ }^{83}$ M. Kiehn, ${ }^{54}$ C. R. Kilby, ${ }^{94}$ E. Kim, ${ }^{164}$ Y. K. Kim, ${ }^{37}$ N. Kimura, ${ }^{95}$ O. M. Kind, ${ }^{19}$ B. T. King, ${ }^{91, a}$ D. Kirchmeier, ${ }^{48}$ J. Kirk, ${ }^{143}$ A. E. Kiryunin, ${ }^{115}$ T. Kishimoto, ${ }^{162}$ D. P. Kisliuk, ${ }^{166}$ V. Kitali, ${ }^{46}$ C. Kitsaki, ${ }^{10}$ O. Kivernyk, ${ }^{24}$ T. Klapdor-Kleingrothaus, ${ }^{52}$ M. Klassen, ${ }^{61 a}$ C. Klein,${ }^{34}$ M. H. Klein, ${ }^{106}$ M. Klein,${ }^{91}$ U. Klein, ${ }^{91}$ K. Kleinknecht, ${ }^{100}$ P. Klimek, ${ }^{121}$ A. Klimentov, ${ }^{29}$ T. Klingl, ${ }^{24}$ T. Klioutchnikova, ${ }^{36}$ F. F. Klitzner, ${ }^{114}$ P. Kluit, ${ }^{120}$ S. Kluth, ${ }^{115}$ E. Kneringer, ${ }^{77}$ E. B. F. G. Knoops,${ }^{102}$ A. Knue, ${ }^{52}$ D. Kobayashi, ${ }^{88}$ T. Kobayashi, ${ }^{162}$ M. Kobel, ${ }^{48}$ M. Kocian,${ }^{152}$ T. Kodama, ${ }^{162}$ P. Kodys, ${ }^{142}$ D. M. Koeck, ${ }^{155}$ P. T. Koenig, ${ }^{24}$ T. Koffas, ${ }^{34}$ N. M. Köhler ${ }^{36}$ M. Kolb, ${ }^{144}$ I. Koletsou, ${ }^{5}$ T. Komarek, ${ }^{130}$ T. Kondo, ${ }^{82}$ K. Köneke, ${ }^{52}$ A. X. Y. Kong, ${ }^{1}$ A. C. König, ${ }^{119}$ T. Kono, ${ }^{126}$ V. Konstantinides, ${ }^{95}$ N. Konstantinidis, ${ }^{95}$ B. Konya, ${ }^{97}$ R. Kopeliansky, ${ }^{66}$ S. Koperny, ${ }^{84 a}$ K. Korcyl, ${ }^{85}$ K. Kordas, ${ }^{161}$ G. Koren, ${ }^{160}$ A. Korn, ${ }^{95}$ I. Korolkov, ${ }^{14}$ E. V. Korolkova, ${ }^{148}$ N. Korotkova, ${ }^{113}$ O. Kortner, ${ }^{115}$ S. Kortner, ${ }^{115}$ V. V. Kostyukhin, ${ }^{148,165}$

A. Kotsokechagia, ${ }^{65}$ A. Kotwal, ${ }^{49}$ A. Koulouris, ${ }^{10}$ A. Kourkoumeli-Charalampidi, ${ }^{71 a, 71 b}$ C. Kourkoumelis, ${ }^{9}$ E. Kourlitis, ${ }^{148}$ V. Kouskoura, ${ }^{29}$ A. B. Kowalewska,${ }^{85}$ R. Kowalewski, ${ }^{175}$ W. Kozanecki, ${ }^{101}$ A. S. Kozhin, ${ }^{123}$ V. A. Kramarenko, ${ }^{113}$ G. Kramberger, ${ }^{92}$ D. Krasnopevtsev, ${ }^{60 a}$ M. W. Krasny, ${ }^{135}$ A. Krasznahorkay, ${ }^{36}$ D. Krauss, ${ }^{115}$ J. A. Kremer, ${ }^{100}$

J. Kretzschmar, ${ }^{91}$ P. Krieger, ${ }^{166}$ F. Krieter, ${ }^{114}$ A. Krishnan, ${ }^{61 \mathrm{~b}}$ K. Krizka, ${ }^{18}$ K. Kroeninger, ${ }^{47}$ H. Kroha, ${ }^{115}$ J. Kroll, ${ }^{140}$ J. Kroll, ${ }^{136}$ K. S. Krowpman, ${ }^{107}$ U. Kruchonak, ${ }^{80}$ H. Krüger, ${ }^{24}$ N. Krumnack, ${ }^{79}$ M. C. Kruse, ${ }^{49}$ J. A. Krzysiak ${ }^{85}$ T. Kubota, ${ }^{105}$ O. Kuchinskaia, ${ }^{165}$ S. Kuday, ${ }^{4 b}$ J. T. Kuechler, ${ }^{46}$ S. Kuehn, ${ }^{36}$ A. Kugel, ${ }^{61 \mathrm{a}}$ T. Kuhl, ${ }^{46}$ V. Kukhtin, ${ }^{80}$ Y. Kulchitsky, ${ }^{108, \text { aa }}$ S. Kuleshov, ${ }^{146 b}$ Y. P. Kulinich, ${ }^{172}$ M. Kuna, ${ }^{58}$ T. Kunigo, ${ }^{86}$ A. Kupco, ${ }^{140}$ T. Kupfer, ${ }^{47}$ O. Kuprash, ${ }^{52}$ H. Kurashige, ${ }^{83}$ L. L. Kurchaninov, ${ }^{167 a}$ Y. A. Kurochkin, ${ }^{108}$ A. Kurova, ${ }^{112}$ M. G. Kurth, ${ }^{15 a, 15 d}$ E. S. Kuwertz, ${ }^{36}$ M. Kuze, ${ }^{164}$ A. K. Kvam, ${ }^{147}$ J. Kvita, ${ }^{130}$ T. Kwan, ${ }^{104}$ L. La Rotonda, ${ }^{41 b, 41 a}$ F. La Ruffa, ${ }^{41 b, 41 a}$ C. Lacasta, ${ }^{173}$ F. Lacava, ${ }^{73 a, 73 b}$ D. P. J. Lack, ${ }^{101}$ H. Lacker, ${ }^{19}$ D. Lacour, ${ }^{135}$ E. Ladygin,${ }^{80}$ R. Lafaye, ${ }^{5}$ B. Laforge, ${ }^{135}$ T. Lagouri, ${ }^{146 \mathrm{~b}}$ S. Lai, ${ }^{53}$ I. K. Lakomiec, ${ }^{84 a}$ S. Lammers, ${ }^{66}$ W. Lampl,${ }^{7}$ C. Lampoudis, ${ }^{161}$ E. Lançon, ${ }^{29}$ U. Landgraf, ${ }^{52}$ M. P. J. Landon, ${ }^{93}$ M. C. Lanfermann, ${ }^{54}$ V. S. Lang, ${ }^{52}$ J. C. Lange, ${ }^{53}$ R. J. Langenberg, ${ }^{103}$ A. J. Lankford, ${ }^{170}$ F. Lanni, ${ }^{29}$ K. Lantzsch, ${ }^{24}$ A. Lanza, ${ }^{71 a}$ A. Lapertosa, ${ }^{55,55 a}$ S. Laplace, ${ }^{135}$ J. F. Laporte, ${ }^{144}$ T. Lari, ${ }^{69 a}$ F. Lasagni Manghi, ${ }^{23 b, 23 a}$ M. Lassnig, ${ }^{36}$ T. S. Lau, ${ }^{63 a}$ A. Laudrain, ${ }^{65}$ A. Laurier, ${ }^{34}$ M. Lavorgna, ${ }^{70 a, 70 b}$ S. D. Lawlor, ${ }^{94}$ M. Lazzaroni,,${ }^{6 a, 69 b}$ B. Le,${ }^{101}$ E. Le Guirriec, ${ }^{102}$ A. Lebedev, ${ }^{79}$ M. LeBlanc, ${ }^{7}$ T. LeCompte, ${ }^{6}$ F. Ledroit-Guillon, ${ }^{58}$ A. C. A. Lee,${ }^{95}$ C. A. Lee, ${ }^{29}$ G. R. Lee ${ }^{17}$ L. Lee, ${ }^{59}$ S. C. Lee, ${ }^{157}$ S. Lee, ${ }^{79}$ B. Lefebvre, ${ }^{167 a}$ H. P. Lefebvre, ${ }^{94}$ M. Lefebvre, ${ }^{175}$ C. Leggett, ${ }^{18}$ K. Lehmann, ${ }^{151}$ N. Lehmann, ${ }^{20}$ G. Lehmann Miotto, ${ }^{36}$ W. A. Leight, ${ }^{46}$ A. Leisos, ${ }^{161, b b}$ M. A. L. Leite, ${ }^{81 d}$ C. E. Leitgeb, ${ }^{114}$ R. Leitner, ${ }^{142}$ D. Lellouch, ${ }^{179, a}$ K. J. C. Leney, ${ }^{42}$ T. Lenz, ${ }^{24}$ R. Leone, ${ }^{7}$ S. Leone, ${ }^{72 a}$ C. Leonidopoulos,${ }^{50}$ A. Leopold,${ }^{135}$ C. Leroy, ${ }^{110}$ R. Les, ${ }^{166}$ C. G. Lester, ${ }^{32}$ M. Levchenko, ${ }^{137}$ J. Levêque, ${ }^{5}$ D. Levin, ${ }^{106}$ L. J. Levinson, ${ }^{179}$ D. J. Lewis, ${ }^{21}$ B. Li, ${ }^{15 b}$ B. Li, ${ }^{106}$ C-Q. Li, ${ }^{60 a}$

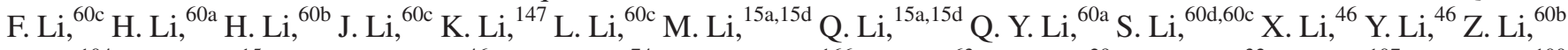
Z. Li, ${ }^{104}$ Z. Liang, ${ }^{15 a}$ M. Liberatore, ${ }^{46}$ B. Liberti, ${ }^{74 a}$ A. Liblong, ${ }^{166}$ K. Lie, ${ }^{63 c}$ S. Lim, ${ }^{29}$ C. Y. Lin, ${ }^{32}$ K. Lin, ${ }^{107}$ T. H. Lin, ${ }^{100}$ 
R. A. Linck, ${ }^{66}$ R. E. Lindley, ${ }^{7}$ J. H. Lindon, ${ }^{21}$ A. Linss, ${ }^{46}$ A. L. Lionti, ${ }^{54}$ E. Lipeles, ${ }^{136}$ A. Lipniacka, ${ }^{17}$ T. M. Liss, ${ }^{172, c c}$ A. Lister, ${ }^{174}$ J. D. Little, ${ }^{8}$ B. Liu, ${ }^{79}$ B. L. Liu, ${ }^{6}$ H. B. Liu, ${ }^{29}$ H. Liu, ${ }^{106}$ J. B. Liu, ${ }^{60 a}$ J. K. K. Liu, ${ }^{37}$ K. Liu, ${ }^{60 d}$ M. Liu,${ }^{60 a}$ P. Liu, ${ }^{15 a}$ Y. Liu, ${ }^{46}$ Y. Liu, ${ }^{15 a, 15 d}$ Y. L. Liu, ${ }^{106}$ Y. W. Liu, ${ }^{60 a}$ M. Livan, ${ }^{71 a, 71 b}$ A. Lleres,${ }^{58}$ J. Llorente Merino, ${ }^{151}$ S. L. Lloyd, ${ }^{93}$ C. Y. Lo, ${ }^{63 \mathrm{~b}}$ E. M. Lobodzinska, ${ }^{46}$ P. Loch, ${ }^{7}$ S. Loffredo, ${ }^{74 a, 74 b}$ T. Lohse, ${ }^{19}$ K. Lohwasser, ${ }^{148}$ M. Lokajicek, ${ }^{140}$ J. D. Long, ${ }^{172}$ R. E. Long, ${ }^{90}$ L. Longo, ${ }^{36}$ K. A. Looper,${ }^{127}$ I. Lopez Paz, ${ }^{101}$ A. Lopez Solis, ${ }^{148}$ J. Lorenz, ${ }^{114}$ N. Lorenzo Martinez, ${ }^{5}$ A. M. Lory, ${ }^{114}$ P. J. Lösel, ${ }^{114}$ A. Lösle, ${ }^{52}$ X. Lou, ${ }^{46}$ X. Lou, ${ }^{15 a}$ A. Lounis, ${ }^{65}$ J. Love, ${ }^{6}$ P. A. Love, ${ }^{90}$ J. J. Lozano Bahilo, ${ }^{173}$ M. Lu, ${ }^{60 a}$ Y. J. Lu, ${ }^{64}$ H. J. Lubatti, ${ }^{147}$ C. Luci, ${ }^{73 a, 73 b}$ A. Lucotte, ${ }^{58}$ C. Luedtke,${ }^{52}$ F. Luehring, ${ }^{66}$ I. Luise, ${ }^{135}$ L. Luminari, ${ }^{73 a}$ B. Lund-Jensen, ${ }^{153}$ M. S. Lutz, ${ }^{160}$ D. Lynn, ${ }^{29}$ H. Lyons, ${ }^{91}$ R. Lysak, ${ }^{140}$ E. Lytken,${ }^{97}$ F. Lyu, ${ }^{15 a}$ V. Lyubushkin, ${ }^{80}$

T. Lyubushkina, ${ }^{80}$ H. Ma, ${ }^{29}$ L. L. Ma ${ }^{60 b}$ Y. Ma,${ }^{95}$ G. Maccarrone, ${ }^{51}$ A. Macchiolo, ${ }^{115}$ C. M. Macdonald, ${ }^{148}$ J. Machado Miguens, ${ }^{136}$ D. Madaffari, ${ }^{173}$ R. Madar, ${ }^{38}$ W. F. Mader, ${ }^{48}$ M. Madugoda Ralalage Don, ${ }^{129}$ N. Madysa, ${ }^{48}$ J. Maeda ${ }^{83}$ T. Maeno, ${ }^{29}$ M. Maerker, ${ }^{48}$ V. Magerl, ${ }^{52}$ N. Magini, ${ }^{79}$ J. Magro, ${ }^{67,67 c, n}$ D. J. Mahon, ${ }^{39}$ C. Maidantchik, ${ }^{81 b}$ T. Maier ${ }^{114}$ A. Maio, ${ }^{139 a, 139 b, 139 d}$ K. Maj, ${ }^{84 a}$ O. Majersky, ${ }^{28 a}$ S. Majewski, ${ }^{131}$ Y. Makida ${ }^{82}$ N. Makovec,${ }^{65}$ B. Malaescu, ${ }^{135}$ Pa. Malecki,${ }^{85}$ V. P. Maleev, ${ }^{137}$ F. Malek,${ }^{58}$ U. Mallik, ${ }^{78}$ D. Malon,${ }^{6}$ C. Malone,${ }^{32}$ S. Maltezos, ${ }^{10}$ S. Malyukov, ${ }^{80}$ J. Mamuzic, ${ }^{173}$ G. Mancini, ${ }^{51}$ I. Mandić, ${ }^{92}$ L. Manhaes de Andrade Filho, ${ }^{81 a}$ I. M. Maniatis, ${ }^{161}$ J. Manjarres Ramos, ${ }^{48}$ K. H. Mankinen, ${ }^{97}$ A. Mann, ${ }^{114}$ A. Manousos, ${ }^{77}$ B. Mansoulie, ${ }^{144}$ I. Manthos,${ }^{161}$ S. Manzoni, ${ }^{120}$ A. Marantis, ${ }^{161}$ G. Marceca, ${ }^{30}$ L. Marchese, ${ }^{134}$ G. Marchiori, ${ }^{135}$ M. Marcisovsky, ${ }^{140}$ L. Marcoccia,${ }^{74 a, 74 b}$ C. Marcon, ${ }^{97}$ C. A. Marin Tobon, ${ }^{36}$ M. Marjanovic, ${ }^{128}$ Z. Marshall, ${ }^{18}$ M. U. F. Martensson, ${ }^{171}$ S. Marti-Garcia, ${ }^{173}$ C. B. Martin, ${ }^{127}$ T. A. Martin, ${ }^{177}$ V. J. Martin,${ }^{50}$ B. Martin dit Latour, ${ }^{17}$ L. Martinelli, ${ }^{75 a, 75 b}$ M. Martinez, ${ }^{14, t}$ P. Martinez Agullo, ${ }^{173}$ V. I. Martinez Outschoorn, ${ }^{103}$ S. Martin-Haugh, ${ }^{143}$ V. S. Martoiu, ${ }^{27 b}$ A. C. Martyniuk, ${ }^{95}$ A. Marzin,${ }^{36}$ S. R. Maschek, ${ }^{115}$ L. Masetti, ${ }^{100}$ T. Mashimo, ${ }^{162}$ R. Mashinistov, ${ }^{111}$ J. Masik, ${ }^{101}$ A. L. Maslennikov, ${ }^{122 b, 122 \mathrm{a}}$ L. Massa, ${ }^{23 b, 23 \mathrm{a}}$ P. Massarotti, ${ }^{70,70 \mathrm{~b}}$ P. Mastrandrea, ${ }^{72 \mathrm{a}, 72 \mathrm{~b}}$ A. Mastroberardino, ${ }^{41 \mathrm{~b}, 41 \mathrm{a}}$ T. Masubuchi, ${ }^{162}$ D. Matakias, ${ }^{29}$ A. Matic, ${ }^{114}$ N. Matsuzawa, ${ }^{162}$ P. Mättig, ${ }^{24} \mathrm{~J}$. Maurer, ${ }^{27 b}$ B. Maček, ${ }^{92}$ D. A. Maximov, ${ }^{122 b, 122 a}$ R. Mazini, ${ }^{157}$ I. Maznas, ${ }^{161}$ S. M. Mazza, ${ }^{145}$ J. P. Mc Gowan, ${ }^{104}$ S. P. Mc Kee, ${ }^{106}$ T. G. McCarthy, ${ }^{115}$ W. P. McCormack,${ }^{18}$ E. F. McDonald, ${ }^{105}$ J. A. Mcfayden, ${ }^{36}$ G. Mchedlidze, ${ }^{158 b}$ M. A. McKay, ${ }^{42}$ K. D. McLean, ${ }^{175}$ S. J. McMahon, ${ }^{143}$ P. C. McNamara, ${ }^{105}$ C. J. McNicol, ${ }^{177}$ R. A. McPherson, ${ }^{175,1}$ J. E. Mdhluli, ${ }^{33 e}$ Z. A. Meadows, ${ }^{103}$ S. Meehan, ${ }^{36}$ T. Megy, ${ }^{38}$ S. Mehlhase, ${ }^{114}$ A. Mehta, ${ }^{91}$ B. Meirose, ${ }^{43}$ D. Melini, ${ }^{159}$ B. R. Mellado Garcia, ${ }^{33 e}$ J. D. Mellenthin, ${ }^{53}$ M. Melo, ${ }^{28 a}$ F. Meloni, ${ }^{46}$ A. Melzer, ${ }^{24}$ S. B. Menary, ${ }^{101}$

E. D. Mendes Gouveia, ${ }^{139 a, 139 \mathrm{e}}$ L. Meng, ${ }^{36}$ X. T. Meng, ${ }^{106}$ S. Menke, ${ }^{115}$ E. Meoni, ${ }^{41 b, 41 a}$ S. Mergelmeyer, ${ }^{19}$

S. A. M. Merkt ${ }^{138}$ C. Merlassino, ${ }^{134}$ P. Mermod, ${ }^{54}$ L. Merola,${ }^{70 a, 70 b}$ C. Meroni, ${ }^{69 a}$ G. Merz,${ }^{106}$ O. Meshkov, ${ }^{113,111}$ J. K. R. Meshreki, ${ }^{150}$ A. Messina, ${ }^{73 a, 73 b}$ J. Metcalfe ${ }^{6}$ A. S. Mete, ${ }^{6}$ C. Meyer, ${ }^{66}$ J-P. Meyer, ${ }^{144}$ H. Meyer Zu Theenhausen, ${ }^{61 a}$ F. Miano, ${ }^{155}$ M. Michetti, ${ }^{19}$ R. P. Middleton, ${ }^{143}$ L. Mijović, ${ }^{50}$ G. Mikenberg, ${ }^{179}$ M. Mikestikova,${ }^{140}$ M. Mikuž,${ }^{92}$ H. Mildner, ${ }^{148}$ M. Milesi, ${ }^{105}$ A. Milic, ${ }^{166}$ C. D. Milke, ${ }^{42}$ D. W. Miller, ${ }^{37}$ A. Milov, ${ }^{179}$ D. A. Milstead,${ }^{45 a, 45 b}$ R. A. Mina, ${ }^{152}$ A. A. Minaenko, ${ }^{123}$ M. Miñano Moya, ${ }^{173}$ I. A. Minashvili, ${ }^{158 b}$ A. I. Mincer ${ }^{125}$ B. Mindur, ${ }^{84 a}$ M. Mineev ${ }^{80}$ Y. Minegishi, ${ }^{162}$ L. M. Mir ${ }^{14}$ M. Mironova, ${ }^{134}$ A. Mirto,${ }^{68 a, 68 b}$ K. P. Mistry, ${ }^{136}$ T. Mitani,${ }^{178}$ J. Mitrevski, ${ }^{114}$ V. A. Mitsou, ${ }^{173}$ M. Mittal,${ }^{60 \mathrm{c}}$ O. Miu, ${ }^{166}$ A. Miucci, ${ }^{20}$ P. S. Miyagawa, ${ }^{148}$ A. Mizukami ${ }^{82}$ J. U. Mjörnmark, ${ }^{97}$ T. Mkrtchyan, ${ }^{61 a}$ M. Mlynarikova, ${ }^{142}$ T. Moa, ${ }^{45 a}, 45 b$ S. Mobius,${ }^{53}$ K. Mochizuki, ${ }^{110}$ P. Mogg, ${ }^{114}$ S. Mohapatra, ${ }^{39}$ R. Moles-Valls, ${ }^{24}$ M. C. Mondragon,${ }^{107}$ K. Mönig, ${ }^{46}$ E. Monnier, ${ }^{102}$ A. Montalbano, ${ }^{151}$ J. Montejo Berlingen, ${ }^{36}$ M. Montella, ${ }^{95}$ F. Monticelli, ${ }^{89}$ S. Monzani, ${ }^{69 a}$ N. Morange ${ }^{65}$ D. Moreno, ${ }^{22 a}$ M. Moreno Llácer, ${ }^{173}$ C. Moreno Martinez, ${ }^{14}$ P. Morettini, ${ }^{55 b}$ M. Morgenstern, ${ }^{159}$ S. Morgenstern, ${ }^{48}$ D. Mori, ${ }^{151}$ M. Morii, ${ }^{59}$ M. Morinaga, ${ }^{178}$ V. Morisbak, ${ }^{133}$ A. K. Morley, ${ }^{36}$ G. Mornacchi, ${ }^{36}$ A. P. Morris, ${ }^{95}$ L. Morvaj, ${ }^{154}$ P. Moschovakos, ${ }^{36}$ B. Moser, ${ }^{120}$ M. Mosidze, ${ }^{158 b}$ T. Moskalets, ${ }^{144}$ H. J. Moss, ${ }^{148}$ J. Moss, ${ }^{31, d d}$ E. J. W. Moyse, ${ }^{103}$ S. Muanza, ${ }^{102}$ J. Mueller ${ }^{138}$ R. S. P. Mueller,${ }^{114}$ D. Muenstermann, ${ }^{90}$ G. A. Mullier, ${ }^{97}$ D. P. Mungo, ${ }^{69 a, 69 b}$ J. L. Munoz Martinez, ${ }^{14}$ F. J. Munoz Sanchez, ${ }^{101}$ P. Murin ${ }^{28 b}$ W. J. Murray, ${ }^{177,143}$ A. Murrone ${ }^{69 a, 69 b}$ M. Muškinja, ${ }^{18}$ C. Mwewa, ${ }^{33 a}$ A. G. Myagkov, ${ }^{123, i}$ A. A. Myers, ${ }^{138}$ J. Myers, ${ }^{131}$ M. Myska, ${ }^{141}$ B. P. Nachman, ${ }^{18}$ O. Nackenhorst, ${ }^{47}$ A. Nag Nag, ${ }^{48}$ K. Nagai, ${ }^{134}$ K. Nagano, ${ }^{82}$ Y. Nagasaka, ${ }^{62}$ J. L. Nagle, ${ }^{29}$ E. Nagy, ${ }^{102}$ A. M. Nairz,${ }^{36}$ Y. Nakahama,${ }^{117}$ K. Nakamura, ${ }^{82}$ T. Nakamura, ${ }^{162}$ H. Nanjo, ${ }^{132}$ F. Napolitano, ${ }^{61 a}$ R. F. Naranjo Garcia, ${ }^{46}$ R. Narayan, ${ }^{42}$ I. Naryshkin, ${ }^{137}$ T. Naumann, ${ }^{46}$ G. Navarro, ${ }^{22 a}$ P. Y. Nechaeva, ${ }^{111}$ F. Nechansky, ${ }^{46}$ T. J. Neep,${ }^{21}$ A. Negri, ${ }^{71 a, 71 b}$ M. Negrini ${ }^{23 b}$ C. Nellist, ${ }^{119}$ M. E. Nelson, ${ }^{45,45 b}$ S. Nemecek, ${ }^{140}$ M. Nessi, ${ }^{36, e e}$ M. S. Neubauer, ${ }^{172}$ F. Neuhaus, ${ }^{100}$ M. Neumann, ${ }^{181}$ R. Newhouse, ${ }^{174}$ P. R. Newman, ${ }^{21}$ C. W. Ng, ${ }^{138}$ Y. S. Ng, ${ }^{19}$ Y.W. Y. Ng, ${ }^{170}$ B. Ngair, ${ }^{35 e}$ H. D. N. Nguyen, ${ }^{102}$ T. Nguyen Manh, ${ }^{110}$ E. Nibigira, ${ }^{38}$ R. B. Nickerson, ${ }^{134}$ R. Nicolaidou ${ }^{144}$ D. S. Nielsen, ${ }^{40}$ J. Nielsen, ${ }^{145}$ N. Nikiforou, ${ }^{11}$ V. Nikolaenko, ${ }^{123, i}$ I. Nikolic-Audit, ${ }^{135}$ K. Nikolopoulos, ${ }^{21}$ P. Nilsson, ${ }^{29}$ H. R. Nindhito, ${ }^{54}$ Y. Ninomiya,${ }^{82}$ A. Nisati, ${ }^{73 a}$ N. Nishu, ${ }^{60 \mathrm{c}}$ R. Nisius, ${ }^{115}$ 
I. Nitsche, ${ }^{47}$ T. Nitta, ${ }^{178}$ T. Nobe, ${ }^{162}$ D. L. Noel,${ }^{32}$ Y. Noguchi,${ }^{86}$ I. Nomidis, ${ }^{135}$ M. A. Nomura, ${ }^{29}$ M. Nordberg, ${ }^{36}$ J. Novak,${ }^{92}$ T. Novak, ${ }^{92}$ O. Novgorodova, ${ }^{48}$ R. Novotny, ${ }^{141}$ L. Nozka, ${ }^{130}$ K. Ntekas, ${ }^{170}$ E. Nurse, ${ }^{95}$ F. G. Oakham, ${ }^{34, d}$ H. Oberlack, ${ }^{115}$ J. Ocariz, ${ }^{135}$ A. Ochi, ${ }^{83}$ I. Ochoa, ${ }^{39}$ J. P. Ochoa-Ricoux, ${ }^{146 a}$ K. O'Connor, ${ }^{26}$ S. Oda, ${ }^{88}$ S. Odaka, ${ }^{82}$ S. Oerdek, ${ }^{53}$ A. Ogrodnik, ${ }^{84 a}$ A. Oh, ${ }^{101}$ S. H. Oh, ${ }^{49}$ C. C. Ohm, ${ }^{153}$ H. Oide, ${ }^{164}$ M. L. Ojeda, ${ }^{166}$ H. Okawa, ${ }^{168}$ Y. Okazaki, ${ }^{86}$ M. W. O’Keefe, ${ }^{91}$ Y. Okumura, ${ }^{162}$ T. Okuyama, ${ }^{82}$ A. Olariu, ${ }^{27 b}$ L. F. Oleiro Seabra, ${ }^{139 a}$ S. A. Olivares Pino, ${ }^{146 a}$ D. Oliveira Damazio, ${ }^{29}$ J. L. Oliver, ${ }^{1}$ M. J. R. Olsson, ${ }^{170}$ A. Olszewski, ${ }^{85}$ J. Olszowska, ${ }^{85}$ D. C. O'Neil, ${ }^{151}$ A. P. O'neill, ${ }^{134}$ A. Onofre, ${ }^{139 a, 139 e}$ P. U. E. Onyisi, ${ }^{11}$ H. Oppen, ${ }^{133}$ R. G. Oreamuno Madriz, ${ }^{121}$ M. J. Oreglia, ${ }^{37}$ G. E. Orellana, ${ }^{89}$ D. Orestano, ${ }^{75 a, 75 b}$ N. Orlando, ${ }^{14}$ R. S. Orr, ${ }^{166}$ V. O’Shea, ${ }^{57}$ R. Ospanov, ${ }^{60 a}$ G. Otero y Garzon, ${ }^{30}$ H. Otono, ${ }^{88}$ P. S. Ott, ${ }^{61 a}$ G. J. Ottino, ${ }^{18}$ M. Ouchrif, ${ }^{35 d}$ J. Ouellette, ${ }^{29}$ F. Ould-Saada, ${ }^{133}$ A. Ouraou, ${ }^{144}$ Q. Ouyang, ${ }^{15 a}$ M. Owen, ${ }^{57}$ R. E. Owen, ${ }^{21}$ V.E. Ozcan, ${ }^{12 \mathrm{c}}$ N. Ozturk,${ }^{8}$ J. Pacalt, ${ }^{130}$ H. A. Pacey, ${ }^{32}$ K. Pachal,${ }^{49}$ A. Pacheco Pages,${ }^{14}$ C. Padilla Aranda,${ }^{14}$ S. Pagan Griso, ${ }^{18}$ M. Paganini, ${ }^{182}$ G. Palacino, ${ }^{66}$ S. Palazzo, ${ }^{50}$ S. Palestini,${ }^{36}$ M. Palka, ${ }^{84 b}$ D. Pallin, ${ }^{38}$ P. Palni, ${ }^{84 a}$ I. Panagoulias, ${ }^{10}$ C. E. Pandini, ${ }^{36}$ J. G. Panduro Vazquez, ${ }^{94}$ P. Pani, ${ }^{46}$ G. Panizzo, ${ }^{67 a, 67 c}$ L. Paolozzi,${ }^{54}$ C. Papadatos, ${ }^{110}$ K. Papageorgiou, ${ }^{9, \mathrm{r}}$ S. Parajuli, ${ }^{42}$ A. Paramonov, ${ }^{6}$ C. Paraskevopoulos, ${ }^{10}$ D. Paredes Hernandez, ${ }^{63 \mathrm{~b}}$ S. R. Paredes Saenz, ${ }^{134}$ B. Parida, ${ }^{165}$ T. H. Park, ${ }^{166}$ A. J. Parker, ${ }^{31}$ M. A. Parker, ${ }^{32}$ F. Parodi, ${ }^{55,55 a}$ E. W. Parrish, ${ }^{121}$ J. A. Parsons, ${ }^{39}$ U. Parzefall, ${ }^{52}$ L. Pascual Dominguez, ${ }^{135}$ V. R. Pascuzzi, ${ }^{18}$ J. M. P. Pasner,${ }^{145}$ F. Pasquali, ${ }^{120}$ E. Pasqualucci, ${ }^{73 a}$ S. Passaggio, ${ }^{55 b}$ F. Pastore,${ }^{94}$ P. Pasuwan, ${ }^{45 a, 45 b}$ S. Pataraia, ${ }^{100}$ J. R. Pater, ${ }^{101}$ A. Pathak, ${ }^{180, e}$ J. Patton, ${ }^{91}$ T. Pauly, ${ }^{36}$ J. Pearkes, ${ }^{152}$ B. Pearson, ${ }^{115}$ M. Pedersen, ${ }^{133}$ L. Pedraza Diaz, ${ }^{119}$ R. Pedro, ${ }^{139 a}$ T. Peiffer, ${ }^{53}$ S. V. Peleganchuk, ${ }^{122 b, 122 a}$ O. Penc, ${ }^{140}$ H. Peng, ${ }^{60 a}$ B. S. Peralva ${ }^{81 a}$ M. M. Perego ${ }^{65}$ A. P. Pereira Peixoto, ${ }^{139 a}$ L. Pereira Sanchez,${ }^{45 a, 45 b}$ D. V. Perepelitsa, ${ }^{29}$ F. Peri, ${ }^{19}$ L. Perini, ${ }^{69 a, 69 b}$ H. Pernegger, ${ }^{36}$ S. Perrella, ${ }^{139 a}$ A. Perrevoort, ${ }^{120}$ K. Peters,${ }^{46}$ R. F. Y. Peters, ${ }^{101}$ B. A. Petersen, ${ }^{36}$ T. C. Petersen, ${ }^{40}$ E. Petit, ${ }^{102}$ A. Petridis, ${ }^{1}$ C. Petridou, ${ }^{161}$ P. Petroff, ${ }^{65}$ F. Petrucci, ${ }^{75 a, 75 b}$ M. Pettee,${ }^{182}$ N. E. Pettersson, ${ }^{103}$ K. Petukhova, ${ }^{142}$ A. Peyaud, ${ }^{144}$ R. Pezoa,${ }^{146 \mathrm{~d}}$ L. Pezzotti, ${ }^{71 \mathrm{a}, 7 \mathrm{~b}}$ T. Pham, ${ }^{105}$ F. H. Phillips, ${ }^{107}$ P. W. Phillips, ${ }^{143}$ M. W. Phipps,${ }^{172}$ G. Piacquadio, ${ }^{154}$ E. Pianori, ${ }^{18}$ A. Picazio, ${ }^{103}$ R. H. Pickles, ${ }^{101}$ R. Piegaia,${ }^{30}$ D. Pietreanu, ${ }^{27 b}$ J. E. Pilcher, ${ }^{37}$ A. D. Pilkington, ${ }^{101}$ M. Pinamonti, ${ }^{67 a, 67 c}$ J. L. Pinfold, ${ }^{3}$ C. Pitman Donaldson, ${ }^{95}$ M. Pitt, ${ }^{160}$ L. Pizzimento, ${ }^{74 a, 74 b}$ M.-A. Pleier, ${ }^{29}$ V. Pleskot, ${ }^{142}$ E. Plotnikova, ${ }^{80}$ P. Podberezko, ${ }^{122 b, 122 a}$ R. Poettgen, ${ }^{97}$ R. Poggi, ${ }^{54}$ L. Poggioli, ${ }^{135}$ I. Pogrebnyak, ${ }^{107}$ D. Pohl,${ }^{24}$ I. Pokharel,${ }^{53}$ G. Polesello, ${ }^{71 a}$ A. Poley, ${ }^{18}$ A. Policicchio, ${ }^{73 a, 73 b}$ R. Polifka, ${ }^{142}$ A. Polini, ${ }^{23 b}$ C. S. Pollard, ${ }^{46}$ V. Polychronakos, ${ }^{29}$ D. Ponomarenko, ${ }^{112}$ L. Pontecorvo, ${ }^{36}$ S. Popa, ${ }^{27 a}$ G. A. Popeneciu, ${ }^{27 d}$ L. Portales, ${ }^{5}$ D. M. Portillo Quintero, ${ }^{58}$ S. Pospisil,${ }^{141}$ K. Potamianos,${ }^{46}$ I. N. Potrap,${ }^{80}$ C. J. Potter, ${ }^{32}$ H. Potti, ${ }^{11}$ T. Poulsen, ${ }^{97}$ J. Poveda, ${ }^{173}$

T. D. Powell, ${ }^{148}$ G. Pownall, ${ }^{46}$ M. E. Pozo Astigarraga, ${ }^{36}$ P. Pralavorio, ${ }^{102}$ S. Prell, ${ }^{79}$ D. Price,${ }^{101}$ M. Primavera, ${ }^{68 a}$ S. Prince, ${ }^{104}$ M. L. Proffitt, ${ }^{147}$ N. Proklova, ${ }^{112}$ K. Prokofiev, ${ }^{63 c}$ F. Prokoshin, ${ }^{80}$ S. Protopopescu, ${ }^{29}$ J. Proudfoot, ${ }^{6}$ M. Przybycien, ${ }^{84 a}$ D. Pudzha, ${ }^{137}$ A. Puri, ${ }^{172}$ P. Puzo, ${ }^{65}$ J. Qian, ${ }^{106}$ Y. Qin, ${ }^{101}$ A. Quadt,${ }^{53}$ M. Queitsch-Maitland, ${ }^{36}$ A. Qureshi, ${ }^{1}$ M. Racko, ${ }^{28 a}$ F. Ragusa, ${ }^{69 a, 69 b}$ G. Rahal,${ }^{98}$ J. A. Raine,${ }^{54}$ S. Rajagopalan,${ }^{29}$ A. Ramirez Morales, ${ }^{93}$ K. Ran, ${ }^{15 a, 15 d}$ T. Rashid, ${ }^{65}$ D. M. Rauch, ${ }^{46}$ F. Rauscher ${ }^{114}$ S. Rave, ${ }^{100}$ B. Ravina, ${ }^{148}$ I. Ravinovich, ${ }^{179}$ J. H. Rawling, ${ }^{101}$ M. Raymond, ${ }^{36}$ A. L. Read, ${ }^{133}$ N. P. Readioff, ${ }^{58}$ M. Reale ${ }^{68 \mathrm{a}, 68 \mathrm{~b}}$ D. M. Rebuzzi, ${ }^{71,71 \mathrm{~b}}$ G. Redlinger, ${ }^{29}$ K. Reeves, ${ }^{43}$ L. Rehnisch, ${ }^{19}$ J. Reichert, ${ }^{136}$ D. Reikher, ${ }^{160}$ A. Reiss,${ }^{100}$ A. Rej, ${ }^{150}$ C. Rembser, ${ }^{36}$ A. Renardi, ${ }^{46}$ M. Renda,${ }^{27 b}$ M. Rescigno, ${ }^{73 a}$ S. Resconi ${ }^{69 a}$ E. D. Resseguie, ${ }^{18}$ S. Rettie, ${ }^{95}$ B. Reynolds, ${ }^{127}$ E. Reynolds, ${ }^{21}$ O. L. Rezanova, ${ }^{122 b, 122 a}$ P. Reznicek ${ }^{142}$ E. Ricci, ${ }^{76 a, 76 b}$ R. Richter, ${ }^{115}$ S. Richter, ${ }^{46}$ E. Richter-Was, ${ }^{84 b}$ O. Ricken, ${ }^{24}$ M. Ridel,${ }^{135}$ P. Rieck, ${ }^{115}$ O. Rifki, ${ }^{46}$ M. Rijssenbeek, ${ }^{154}$ A. Rimoldi, ${ }^{71 a, 71 b}$ M. Rimoldi, ${ }^{46}$ L. Rinaldi, ${ }^{23 b}$ G. Ripellino, ${ }^{153}$ I. Riu, ${ }^{14}$ P. Rivadeneira, ${ }^{46}$ J. C. Rivera Vergara, ${ }^{175}$ F. Rizatdinova, ${ }^{129}$ E. Rizvi, ${ }^{93}$ C. Rizzi, ${ }^{36}$ R. T. Roberts, ${ }^{101}$ S. H. Robertson, ${ }^{104,1}$ M. Robin, ${ }^{46}$ D. Robinson, ${ }^{32}$ C. M. Robles Gajardo, ${ }^{146 \mathrm{~d}}$ M. Robles Manzano, ${ }^{100}$ A. Robson, ${ }^{57}$ A. Rocchi, ${ }^{74 a, 74 b}$ E. Rocco, ${ }^{100}$ C. Roda ${ }^{72 a, 72 b}$ S. Rodriguez Bosca, ${ }^{173}$ D. Rodriguez Rodriguez,${ }^{173}$ A. M. Rodríguez Vera, ${ }^{167 b}$ S. Roe ${ }^{36}$ O. Røhne, ${ }^{133}$ R. Röhrig, ${ }^{115}$ R. A. Rojas, ${ }^{146 \mathrm{~d}}$ B. Roland ${ }^{52}$ C. P. A. Roland, ${ }^{66}$ J. Roloff, ${ }^{29}$ A. Romaniouk, ${ }^{112}$ M. Romano, ${ }^{23 b, 23 a}$ N. Rompotis, ${ }^{91}$ M. Ronzani, ${ }^{125}$ L. Roos, ${ }^{135}$ S. Rosati, ${ }^{73 a}$ G. Rosin,,${ }^{103}$ B. J. Rosser, ${ }^{136}$ E. Rossi ${ }^{46}$ E. Rossi, ${ }^{75 a, 75 b}$ E. Rossi ${ }^{70 a, 70 b}$ L. P. Rossi, ${ }^{55 b}$ L. Rossini, ${ }^{69 a, 69 b}$ R. Rosten, ${ }^{14}$ M. Rotaru, ${ }^{27 b}$ B. Rottler, ${ }^{52}$ D. Rousseau, ${ }^{65}$ G. Rovelli, ${ }^{71 a, 71 b}$ A. Roy, ${ }^{11}$ D. Roy ${ }^{33 e}$ A. Rozanov, ${ }^{102}$ Y. Rozen, ${ }^{159}$ X. Ruan, ${ }^{33 e}$ F. Rühr, ${ }^{52}$ A. Ruiz-Martinez, ${ }^{173}$ A. Rummler, ${ }^{36}$ Z. Rurikova ${ }^{52}$ N. A. Rusakovich, ${ }^{80}$ H. L. Russell, ${ }^{104}$ L. Rustige, ${ }^{38,47}$ J. P. Rutherfoord, ${ }^{7}$ E. M. Rüttinger, ${ }^{148}$ M. Rybar, ${ }^{39}$ G. Rybkin, ${ }^{65}$ E. B. Rye ${ }^{133}$ A. Ryzhov, ${ }^{123}$ J. A. Sabater Iglesias, ${ }^{46}$ P. Sabatini, ${ }^{53}$ S. Sacerdoti, ${ }^{65}$ H. F-W. Sadrozinski ${ }^{145}$ R. Sadykov, ${ }^{80}$ F. Safai Tehrani, ${ }^{73 a}$ B. Safarzadeh Samani, ${ }^{155}$ M. Safdari, ${ }^{152}$ P. Saha, ${ }^{121}$ S. Saha,${ }^{104}$ M. Sahinsoy, ${ }^{61 a}$ A. Sahu, ${ }^{181}$ M. Saimpert, ${ }^{36}$ M. Saito, ${ }^{162}$ T. Saito, ${ }^{162}$ H. Sakamoto, ${ }^{162}$ D. Salamani,${ }^{54}$ G. Salamanna, ${ }^{75 a, 75 b}$ J. E. Salazar Loyola, ${ }^{146 \mathrm{~d}}$ A. Salnikov, ${ }^{152}$ J. Salt, ${ }^{173}$ A. Salvador Salas, ${ }^{14}$ D. Salvatore, ${ }^{41 \mathrm{~b}, 41 \mathrm{a}}$ F. Salvatore, ${ }^{155}$ A. Salvucci, ${ }^{63 a, 63 \mathrm{~b}, 63 \mathrm{c}}$ A. Salzburger, ${ }^{36}$ 
J. Samarati, ${ }^{36}$ D. Sammel, ${ }^{52}$ D. Sampsonidis, ${ }^{161}$ D. Sampsonidou, ${ }^{161}$ J. Sánchez, ${ }^{173}$ A. Sanchez Pineda,${ }^{67 a, 36,67 c}$ H. Sandaker, ${ }^{133}$ C. O. Sander, ${ }^{46}$ I. G. Sanderswood, ${ }^{90}$ M. Sandhoff, ${ }^{181}$ C. Sandoval, ${ }^{22 a}$ D. P. C. Sankey, ${ }^{143}$ M. Sannino, ${ }^{55 b, 55 a}$ Y. Sano, ${ }^{117}$ A. Sansoni,${ }^{51}$ C. Santoni,${ }^{38}$ H. Santos, ${ }^{139 a, 139 b}$ S. N. Santpur, ${ }^{18}$ A. Santra, ${ }^{173}$ A. Sapronov, ${ }^{80}$ J. G. Saraiva, ${ }^{139 a, 139 d}$ O. Sasaki, ${ }^{82}$ K. Sato, ${ }^{168}$ F. Sauerburger, ${ }^{52}$ E. Sauvan, ${ }^{5}$ P. Savard, ${ }^{166, d}$ R. Sawada, ${ }^{162}$ C. Sawyer, ${ }^{143}$ L. Sawyer, ${ }^{96, f f}$ C. Sbarra, ${ }^{23 b}$ A. Sbrizzi, ${ }^{23 a}$ T. Scanlon, ${ }^{95}$ J. Schaarschmidt, ${ }^{147}$ P. Schacht, ${ }^{115}$ B. M. Schachtner, ${ }^{114}$ D. Schaefer, ${ }^{37}$ L. Schaefer, ${ }^{136}$ J. Schaeffer, ${ }^{100}$ S. Schaepe, ${ }^{36}$ U. Schäfer, ${ }^{100}$ A. C. Schaffer, ${ }^{65}$ D. Schaile, ${ }^{114}$ R. D. Schamberger, ${ }^{154}$ E. Schanet, ${ }^{114}$ N. Scharmberg, ${ }^{101}$ V. A. Schegelsky, ${ }^{137}$ D. Scheirich, ${ }^{142}$ F. Schenck, ${ }^{19}$ M. Schernau, ${ }^{170}$ C. Schiavi, ${ }^{55 b, 55 a}$ L. K. Schildgen, ${ }^{24}$ Z. M. Schillaci, ${ }^{26}$ E. J. Schioppa, ${ }^{68 a, 68 b}$ M. Schioppa, ${ }^{41 b, 41 a}$ K. E. Schleicher, ${ }^{52}$ S. Schlenker, ${ }^{36}$ K. R. Schmidt-Sommerfeld, ${ }^{115}$ K. Schmieden, ${ }^{36}$ C. Schmitt, ${ }^{100}$ S. Schmitt, ${ }^{46}$ S. Schmitz, ${ }^{100}$ J. C. Schmoeckel, ${ }^{46}$ L. Schoeffel, ${ }^{144}$ A. Schoening, ${ }^{61 b}$ P. G. Scholer, ${ }^{52}$ E. Schopf, ${ }^{134}$ M. Schott, ${ }^{100}$ J. F. P. Schouwenberg, ${ }^{119}$ J. Schovancova, ${ }^{36}$ S. Schramm, ${ }^{54}$ F. Schroeder, ${ }^{181}$ A. Schulte, ${ }^{100}$ H-C. Schultz-Coulon, ${ }^{61 a}$ M. Schumacher, ${ }^{52}$ B. A. Schumm, ${ }^{145} \mathrm{Ph}$. Schune, ${ }^{144}$ A. Schwartzman, ${ }^{152}$

T. A. Schwarz, ${ }^{106}$ Ph. Schwemling, ${ }^{144}$ R. Schwienhorst, ${ }^{107}$ A. Sciandra, ${ }^{145}$ G. Sciolla,${ }^{26}$ M. Scodeggio, ${ }^{46}$ M. Scornajenghi, ${ }^{41 b, 41 a}$ F. Scuri, ${ }^{72 a}$ F. Scutti, ${ }^{105}$ L. M. Scyboz, ${ }^{115}$ C. D. Sebastiani, ${ }^{73 a, 73 b}$ P. Seema, ${ }^{19}$ S. C. Seidel, ${ }^{118}$ A. Seiden, ${ }^{145}$ B. D. Seidlitz, ${ }^{29}$ T. Seiss,${ }^{37}$ C. Seitz ${ }^{46}$ J. M. Seixas, ${ }^{81 b}$ G. Sekhniaidze, ${ }^{70 a}$ S. J. Sekula, ${ }^{42}$ N. Semprini-Cesari, ${ }^{23 b, 23 a}$ S. Sen, ${ }^{49}$ C. Serfon, ${ }^{29}$ L. Serin, ${ }^{65}$ L. Serkin, ${ }^{67 a, 67 b}$ M. Sessa, ${ }^{60 a}$ H. Severini, ${ }^{128}$ S. Sevova, ${ }^{152}$ F. Sforza, ${ }^{55 b, 55 a}$ A. Sfyrla, ${ }^{54}$ E. Shabalina, ${ }^{53}$ J. D. Shahinian, ${ }^{145}$ N. W. Shaikh,${ }^{45 a, 45 b}$ D. Shaked Renous, ${ }^{179}$ L. Y. Shan, ${ }^{15 a}$ M. Shapiro, ${ }^{18}$ A. Sharma, ${ }^{134}$ A. S. Sharma, ${ }^{1}$ P. B. Shatalov, ${ }^{124}$ K. Shaw, ${ }^{155}$ S. M. Shaw, ${ }^{101}$ M. Shehade, ${ }^{179}$ Y. Shen, ${ }^{128}$ A. D. Sherman, ${ }^{25}$ P. Sherwood, ${ }^{95}$ L. Shi, ${ }^{157}$ S. Shimizu, ${ }^{82}$ C. O. Shimmin, ${ }^{182}$ Y. Shimogama, ${ }^{178}$ M. Shimojima, ${ }^{116}$ I. P. J. Shipsey, ${ }^{134}$ S. Shirabe, ${ }^{164}$ M. Shiyakova, ${ }^{80, g g}$ J. Shlomi, ${ }^{179}$ A. Shmeleva, ${ }^{111}$ M. J. Shochet, ${ }^{37}$ J. Shojaii, ${ }^{105}$ D. R. Shope, ${ }^{128}$ S. Shrestha, ${ }^{127}$ E. M. Shrif, ${ }^{33 \mathrm{e}}$ E. Shulga, ${ }^{179}$ P. Sicho, ${ }^{140}$ A. M. Sickles, ${ }^{172}$ E. Sideras Haddad, ${ }^{33 e}$ O. Sidiropoulou, ${ }^{36}$ A. Sidoti, ${ }^{23 b, 23 a}$ F. Siegert, ${ }^{48}$ Dj. Sijacki, ${ }^{16}$ M. Silva Jr., ${ }^{180}$ M. V. Silva Oliveira, ${ }^{81 a}$ S. B. Silverstein, ${ }^{45 a}$ S. Simion, ${ }^{65}$ R. Simoniello, ${ }^{100}$ C. J. Simpson-allsop, ${ }^{21}$ S. Simsek, ${ }^{12 b}$ P. Sinervo, ${ }^{166}$ V. Sinetckii, ${ }^{13}$ S. Singh, ${ }^{151}$ M. Sioli, ${ }^{23 b, 23 a}$

I. Siral, ${ }^{131}$ S. Yu. Sivoklokov, ${ }^{113}$ J. Sjölin, ${ }^{45 a, 45 b}$ A. Skaf, ${ }^{53}$ E. Skorda, ${ }^{97}$ P. Skubic, ${ }^{128}$ M. Slawinska, ${ }^{85}$ K. Sliwa, ${ }^{169}$ R. Slovak, ${ }^{142}$ V. Smakhtin, ${ }^{179}$ B. H. Smart, ${ }^{143}$ J. Smiesko, ${ }^{28 b}$ N. Smirnov, ${ }^{112}$ S. Yu. Smirnov, ${ }^{112}$ Y. Smirnov, ${ }^{112}$ L. N. Smirnova, ${ }^{13, \text { hh }}$ O. Smirnova, ${ }^{97}$ J. W. Smith, ${ }^{53}$ M. Smizanska, ${ }^{90}$ K. Smolek, ${ }^{141}$ A. Smykiewicz, ${ }^{85}$ A. A. Snesarev, ${ }^{111}$ H. L. Snoek, ${ }^{120}$ I. M. Snyder, ${ }^{131}$ S. Snyder, ${ }^{29}$ R. Sobie, ${ }^{175,1}$ A. Soffer,${ }^{160}$ A. Søgaard, ${ }^{50}$ F. Sohns, ${ }^{53}$ C. A. Solans Sanchez, ${ }^{36}$ E. Yu. Soldatov, ${ }^{112}$ U. Soldevila, ${ }^{173}$ A. A. Solodkov, ${ }^{123}$ A. Soloshenko, ${ }^{80}$ O. V. Solovyanov, ${ }^{123}$ V. Solovyev, ${ }^{137}$ P. Sommer, ${ }^{148}$

H. Son, ${ }^{169}$ W. Song, ${ }^{143}$ W. Y. Song ${ }^{167 b}$ A. Sopczak, ${ }^{141}$ A. L. Sopio, ${ }^{95}$ F. Sopkova, ${ }^{28 b}$ C. L. Sotiropoulou, ${ }^{72 a, 72 b}$ S. Sottocornola ${ }^{71 \mathrm{a}, 71 \mathrm{~b}}$ R. Soualah, ${ }^{67 \mathrm{a}, 67 \mathrm{c}, \mathrm{ii}}$ A. M. Soukharev, ${ }^{122 \mathrm{~b}, 122 \mathrm{a}}$ D. South, ${ }^{46}$ S. Spagnolo, ${ }^{68 \mathrm{a}, 68 \mathrm{~b}}$ M. Spalla, ${ }^{115}$ M. Spangenberg, ${ }^{177}$ F. Spanò, ${ }^{94}$ D. Sperlich,${ }^{52}$ T. M. Spieker, ${ }^{61 a}$ G. Spigo, ${ }^{36}$ M. Spina ${ }^{155}$ D. P. Spiteri, ${ }^{57}$ M. Spousta ${ }^{142}$ A. Stabile, ${ }^{69 a, 69 b}$ B. L. Stamas, ${ }^{121}$ R. Stamen, ${ }^{61 a}$ M. Stamenkovic, ${ }^{120}$ E. Stanecka, ${ }^{85}$ B. Stanislaus, ${ }^{134}$ M. M. Stanitzki, ${ }^{46}$ M. Stankaityte, ${ }^{134}$ B. Stapf,${ }^{120}$ E. A. Starchenko, ${ }^{123}$ G. H. Stark, ${ }^{145}$ J. Stark,${ }^{58}$ P. Staroba, ${ }^{140}$ P. Starovoitov, ${ }^{61 a}$ S. Stärz, ${ }^{104}$ R. Staszewski, ${ }^{85}$ G. Stavropoulos, ${ }^{44}$ M. Stegler, ${ }^{46}$ P. Steinberg, ${ }^{29}$ A. L. Steinhebel, ${ }^{131}$ B. Stelzer, ${ }^{151}$ H. J. Stelzer, ${ }^{138}$ O. Stelzer-Chilton, ${ }^{167 a}$ H. Stenzel, ${ }^{56}$ T. J. Stevenson, ${ }^{155}$ G. A. Stewart ${ }^{36}$ M. C. Stockton, ${ }^{36}$ G. Stoicea, ${ }^{27 b}$ M. Stolarski, ${ }^{139 a}$ S. Stonjek, ${ }^{115}$ A. Straessner, ${ }^{48}$ J. Strandberg, ${ }^{153}$ S. Strandberg, ${ }^{45 a, 45 b}$ M. Strauss, ${ }^{128}$ T. Strebler, ${ }^{102}$ P. Strizenec, ${ }^{28 b}$ R. Ströhmer, ${ }^{176}$ D. M. Strom, ${ }^{131}$ R. Stroynowski, ${ }^{42}$ A. Strubig, ${ }^{50}$ S. A. Stucci, ${ }^{29}$ B. Stugu, ${ }^{17}$ J. Stupak, ${ }^{128}$ N. A. Styles, ${ }^{46}$ D. Su, ${ }^{152}$ W. Su, ${ }^{60 c, 147}$ S. Suchek, ${ }^{61 a}$ V. V. Sulin, ${ }^{111}$ M. J. Sullivan, ${ }^{91}$ D. M. S. Sultan,${ }^{54}$ S. Sultansoy, ${ }^{4 c}$ T. Sumida, ${ }^{86}$ S. Sun, ${ }^{106}$ X. Sun, ${ }^{101}$ K. Suruliz, ${ }^{155}$ C. J. E. Suster, ${ }^{156}$ M. R. Sutton, ${ }^{155}$ S. Suzuki, ${ }^{82}$ M. Svatos,${ }^{140}$ M. Swiatlowski, ${ }^{167 a}$ S. P. Swift, ${ }^{2}$ T. Swirski, ${ }^{176}$ A. Sydorenko, ${ }^{100}$ I. Sykora, ${ }^{28 a}$ M. Sykora, ${ }^{142}$ T. Sykora, ${ }^{142}$ D. Ta, ${ }^{100}$ K. Tackmann, ${ }^{46, j j}$ J. Taenzer, ${ }^{160}$ A. Taffard ${ }^{170}$ R. Tafirout, ${ }^{167 a}$ R. Takashima ${ }^{87}$ K. Takeda, ${ }^{83}$ T. Takeshita ${ }^{149}$ E. P. Takeva ${ }^{50}$ Y. Takubo, ${ }^{82}$ M. Talby, ${ }^{102}$ A. A. Talyshev, ${ }^{122 b, 122 a}$ K. C. Tam, ${ }^{63 b}$ N. M. Tamir,${ }^{160}$ J. Tanaka, ${ }^{162}$ R. Tanaka,${ }^{65}$ S. Tapia Araya, ${ }^{172}$ S. Tapprogge, ${ }^{100}$ A. Tarek Abouelfadl Mohamed, ${ }^{107}$ S. Tarem, ${ }^{159}$ K. Tariq, ${ }^{60 b}$ G. Tarna, ${ }^{27 b, k k}$ G. F. Tartarelli, ${ }^{69 a}$ P. Tas, ${ }^{142}$ M. Tasevsky, ${ }^{140}$ T. Tashiro, ${ }^{86}$ E. Tassi, ${ }^{41 \mathrm{~b}, 41 \mathrm{a}}$ A. Tavares Delgado, ${ }^{139 \mathrm{a}}$ Y. Tayalati, ${ }^{35 \mathrm{e}}$ A. J. Taylor, ${ }^{50}$ G. N. Taylor, ${ }^{105}$ W. Taylor, ${ }^{167 \mathrm{~b}}$ H. Teagle,${ }^{91}$ A. S. Tee, ${ }^{90}$ R. Teixeira De Lima, ${ }^{152}$ P. Teixeira-Dias, ${ }^{94}$ H. Ten Kate, ${ }^{36}$ J. J. Teoh,${ }^{120}$ S. Terada,${ }^{82}$ K. Terashi, ${ }^{162}$ J. Terron, ${ }^{99}$ S. Terzo, ${ }^{14}$ M. Testa, ${ }^{51}$ R. J. Teuscher,${ }^{166,1}$ S. J. Thais,${ }^{182}$ N. Themistokleous, ${ }^{50}$ T. Theveneaux-Pelzer, ${ }^{46}$ F. Thiele, ${ }^{40}$ D. W. Thomas, ${ }^{94}$ J. O. Thomas, ${ }^{42}$ J. P. Thomas, ${ }^{21}$ E. A. Thompson, ${ }^{46}$ P. D. Thompson, ${ }^{21}$ E. Thomson, ${ }^{136}$ E. J. Thorpe, ${ }^{93}$ R. E. Ticse Torres, ${ }^{53}$ V. O. Tikhomirov ${ }^{111,11}$ Yu. A. Tikhonov, ${ }^{122 b, 122 a}$ S. Timoshenko, ${ }^{112}$ P. Tipton, ${ }^{182}$ S. Tisserant, ${ }^{102}$ K. Todome ${ }^{23 b, 23 a}$ S. Todorova-Nova, ${ }^{142}$ S. Todt,${ }^{48}$ J. Tojo, ${ }^{88}$ S. Tokár ${ }^{28 a}$ K. Tokushuku, ${ }^{82}$ E. Tolley, ${ }^{127}$ R. Tombs, ${ }^{32}$ K. G. Tomiwa, ${ }^{33 e}$ M. Tomoto, ${ }^{117}$ L. Tompkins, ${ }^{152}$ P. Tornambe, ${ }^{103}$ E. Torrence, ${ }^{131}$ H. Torres,${ }^{48}$ E. Torró Pastor, ${ }^{147}$ 
C. Tosciri, ${ }^{134}$ J. Toth, ${ }^{102, \mathrm{~mm}}$ D. R. Tovey, ${ }^{148}$ A. Traeet, ${ }^{17}$ C. J. Treado, ${ }^{125}$ T. Trefzger, ${ }^{176}$ F. Tresoldi, ${ }^{155}$ A. Tricoli, ${ }^{29}$ I. M. Trigger, ${ }^{167 a}$ S. Trincaz-Duvoid, ${ }^{135}$ D. A. Trischuk, ${ }^{174}$ W. Trischuk, ${ }^{166}$ B. Trocmé, ${ }^{58}$ A. Trofymov, ${ }^{65}$ C. Troncon, ${ }^{69 a}$ F. Trovato, ${ }^{155}$ L. Truong, ${ }^{33 c}$ M. Trzebinski, ${ }^{85}$ A. Trzupek, ${ }^{85}$ F. Tsai, ${ }^{46}$ J. C-L. Tseng, ${ }^{134}$ P. V. Tsiareshka, ${ }^{108, \text { aa }}$ A. Tsirigotis, ${ }^{161, b b}$ V. Tsiskaridze, ${ }^{154}$ E. G. Tskhadadze, ${ }^{158 a}$ M. Tsopoulou, ${ }^{161}$ I. I. Tsukerman, ${ }^{124}$ V. Tsulaia, ${ }^{18}$ S. Tsuno, ${ }^{82}$ D. Tsybychev, ${ }^{154} \mathrm{Y}$. Tu, ${ }^{63 \mathrm{~b}}$ A. Tudorache, ${ }^{27 \mathrm{~b}} \mathrm{~V}$. Tudorache, ${ }^{27 \mathrm{~b}}$ T. T. Tulbure, ${ }^{27 \mathrm{a}}$ A. N. Tuna, ${ }^{59} \mathrm{~S}$. Turchikhin,${ }^{80}$ D. Turgeman, ${ }^{179}$ I. Turk Cakir, ${ }^{4 b, n n}$ R. J. Turner, ${ }^{21}$ R. Turra, ${ }^{69 a}$ P. M. Tuts, ${ }^{39}$ S. Tzamarias, ${ }^{161}$ E. Tzovara, ${ }^{100}$ G. Ucchielli, ${ }^{47}$ K. Uchida, ${ }^{162}$ F. Ukegawa, ${ }^{168}$ G. Unal ${ }^{36}$ A. Undrus, ${ }^{29}$ G. Unel, ${ }^{170}$ F. C. Ungaro, ${ }^{105}$ Y. Unno,${ }^{82}$ K. Uno, ${ }^{162}$ J. Urban ${ }^{28 b}$ P. Urquijo, ${ }^{105}$ G. Usai ${ }^{8}$ Z. Uysal, ${ }^{12 \mathrm{~d}}$ V. Vacek, ${ }^{141}$ B. Vachon, ${ }^{104}$ K. O. H. Vadla, ${ }^{133}$ A. Vaidya, ${ }^{95}$ C. Valderanis, ${ }^{114}$ E. Valdes Santurio, ${ }^{45 a, 45 b}$ M. Valente, ${ }^{54}$ S. Valentinetti, ${ }^{23 b, 23 a}$ A. Valero, ${ }^{173}$ L. Valéry,${ }^{46}$ R. A. Vallance, ${ }^{21}$ A. Vallier,${ }^{36}$ J. A. Valls Ferrer, ${ }^{173}$ T. R. Van Daalen, ${ }^{14}$ P. Van Gemmeren, ${ }^{6}$ I. Van Vulpen, ${ }^{120}$ M. Vanadia, ${ }^{74 a, 74 b}$ W. Vandelli, ${ }^{36}$ M. Vandenbroucke, ${ }^{144}$ E. R. Vandewall, ${ }^{129}$ A. Vaniachine, ${ }^{165}$ D. Vannicola, ${ }^{73 a, 73 b}$ R. Vari, ${ }^{73 a}$ E. W. Varnes, ${ }^{7}$ C. Varni, ${ }^{55 b, 55 a}$ T. Varol, ${ }^{157}$ D. Varouchas, ${ }^{65}$ K. E. Varvell, ${ }^{156}$ M. E. Vasile, ${ }^{27 b}$ G. A. Vasquez,${ }^{175}$ F. Vazeille, ${ }^{38}$ D. Vazquez Furelos, ${ }^{14}$ T. Vazquez Schroeder, ${ }^{36}$ J. Veatch, ${ }^{53}$ V. Vecchio, ${ }^{101}$ M. J. Veen, ${ }^{120}$ L. M. Veloce, ${ }^{166}$ F. Veloso, ${ }^{139 a, 139 c}$ S. Veneziano, ${ }^{73 a}$ A. Ventura, ${ }^{68 a, 68 b}$ N. Venturi, ${ }^{36}$ A. Verbytskyi, ${ }^{115}$ V. Vercesi, ${ }^{71 a}$ M. Verducci,${ }^{72 a, 72 b}$ C. M. Vergel Infante, ${ }^{79}$ C. Vergis, ${ }^{24}$ W. Verkerke, ${ }^{120}$ A. T. Vermeulen, ${ }^{120}$ J. C. Vermeulen, ${ }^{120}$ C. Vernieri, ${ }^{152}$ M. C. Vetterli, ${ }^{151, d}$ N. Viaux Maira, ${ }^{146 \mathrm{~d}}$ T. Vickey, ${ }^{148}$ O. E. Vickey Boeriu, ${ }^{148}$ G. H. A. Viehhauser, ${ }^{134}$ L. Vigani, ${ }^{61 b}$ M. Villa, ${ }^{23 b, 23 a}$ M. Villaplana Perez, ${ }^{3}$ E. M. Villhauer, ${ }^{50}$ E. Vilucchi, ${ }^{51}$ M. G. Vincter, ${ }^{34}$ G. S. Virdee,${ }^{21}$ A. Vishwakarma, ${ }^{46}$ C. Vittori ${ }^{23 b, 23 a}$ I. Vivarelli, ${ }^{155}$ M. Vogel, ${ }^{181}$ P. Vokac, ${ }^{141}$ S. E. von Buddenbrock, ${ }^{33 e}$ E. Von Toerne, ${ }^{24}$ V. Vorobel, ${ }^{142}$ K. Vorobev, ${ }^{112}$ M. Vos, ${ }^{173}$ J. H. Vossebeld, ${ }^{91}$ M. Vozak, ${ }^{101}$

N. Vranjes, ${ }^{16}$ M. Vranjes Milosavljevic, ${ }^{16}$ V. Vrba, ${ }^{141}$ M. Vreeswijk, ${ }^{120}$ R. Vuillermet, ${ }^{36}$ I. Vukotic,${ }^{37}$ S. Wada, ${ }^{168}$ P. Wagner, ${ }^{24}$ W. Wagner, ${ }^{181}$ J. Wagner-Kuhr, ${ }^{114}$ S. Wahdan, ${ }^{181}$ H. Wahlberg, ${ }^{89}$ R. Wakasa, ${ }^{168}$ V. M. Walbrecht,,${ }^{15}$ J. Walder,${ }^{90}$ R. Walker, ${ }^{114}$ S. D. Walker, ${ }^{94}$ W. Walkowiak, ${ }^{150}$ V. Wallangen,${ }^{45 a, 45 b}$ A. M. Wang, ${ }^{59}$ A. Z. Wang ${ }^{180}$ C. Wang, ${ }^{60 \mathrm{c}}$ F. Wang, ${ }^{180}$ H. Wang, ${ }^{18}$ H. Wang, ${ }^{3}$ J. Wang, ${ }^{63 a}$ J. Wang, ${ }^{61 b}$ P. Wang, ${ }^{42}$ Q. Wang, ${ }^{128}$ R.-J. Wang, ${ }^{100}$ R. Wang, ${ }^{60 a}$ R. Wang, ${ }^{6}$ S. M. Wang, ${ }^{157}$ W. T. Wang, ${ }^{60 \mathrm{a}}$ W. Wang, ${ }^{15 \mathrm{c}}$ W. X. Wang, ${ }^{60 \mathrm{a}}$ Y. Wang, ${ }^{60 \mathrm{a}}$ Z. Wang, ${ }^{60 \mathrm{c}}$ C. Wanotayaroj, ${ }^{46}$ A. Warburton, ${ }^{104}$ C. P. Ward, ${ }^{32}$

D. R. Wardrope, ${ }^{95}$ N. Warrack,${ }^{57}$ A. Washbrook, ${ }^{50}$ A. T. Watson, ${ }^{21}$ M. F. Watson, ${ }^{21}$ G. Watts, ${ }^{147}$ B. M. Waugh ${ }^{95}$

A. F. Webb, ${ }^{11}$ C. Weber, ${ }^{29}$ M. S. Weber, ${ }^{20}$ S. A. Weber, ${ }^{34}$ S. M. Weber,${ }^{61 a}$ A. R. Weidberg, ${ }^{134}$ J. Weingarten, ${ }^{47}$ M. Weirich, ${ }^{100}$

C. Weiser, ${ }^{52}$ P. S. Wells, ${ }^{36}$ T. Wenaus, ${ }^{29}$ T. Wengler, ${ }^{36}$ S. Wenig,${ }^{36}$ N. Wermes,${ }^{24}$ M. D. Werner, ${ }^{79}$ M. Wessels, ${ }^{61 a}$ T. D. Weston, ${ }^{20}$ K. Whalen, ${ }^{131}$ N. L. Whallon, ${ }^{147}$ A. M. Wharton, ${ }^{90}$ A. S. White, ${ }^{106}$ A. White, ${ }^{8}$ M. J. White, ${ }^{1}$ D. Whiteson, ${ }^{170}$ B. W. Whitmore ${ }^{90}$ W. Wiedenmann, ${ }^{180}$ C. Wiel,${ }^{48}$ M. Wielers, ${ }^{143}$ N. Wieseotte,${ }^{100}$ C. Wiglesworth, ${ }^{40}$

L. A. M. Wiik-Fuchs, ${ }^{52}$ H. G. Wilkens, ${ }^{36}$ L. J. Wilkins, ${ }^{94}$ H. H. Williams, ${ }^{136}$ S. Williams,${ }^{32}$ C. Willis, ${ }^{107}$ S. Willocq,${ }^{103}$ P. J. Windischhofer, ${ }^{134}$ I. Wingerter-Seez, ${ }^{5}$ E. Winkels, ${ }^{155}$ F. Winklmeier, ${ }^{131}$ B. T. Winter, ${ }^{52}$ M. Wittgen, ${ }^{152}$ M. Wobisch, ${ }^{96}$ A. Wolf, ${ }^{100}$ T. M. H. Wolf, ${ }^{120}$ R. Wolff, ${ }^{102}$ R. Wölker,${ }^{134}$ J. Wollrath,${ }^{52}$ M. W. Wolter, ${ }^{85}$ H. Wolters, ${ }^{139 a, 139 c}$ V. W. S. Wong, ${ }^{174}$ N. L. Woods, ${ }^{145}$ S. D. Worm, ${ }^{46}$ B. K. Wosiek, ${ }^{85}$ K. W. Woźniak, ${ }^{85}$ K. Wraight,${ }^{57}$ S. L. Wu, ${ }^{180}$ X. Wu, ${ }^{54}$ Y. Wu, ${ }^{60 a}$ J. Wuerzinger, ${ }^{134}$ T. R. Wyatt, ${ }^{101}$ B. M. Wynne, ${ }^{50}$ S. Xella, ${ }^{40}$ Z. Xi, ${ }^{106}$ L. Xia, ${ }^{177}$ X. Xiao, ${ }^{106}$ X. Xie, ${ }^{60 a}$ I. Xiotidis, ${ }^{155}$ D. Xu ${ }^{15 a}$ H. Xu ${ }^{60 a}$ H. Xu, ${ }^{60 a}$ L. Xu, ${ }^{29}$ T. Xu, ${ }^{144}$ W. Xu,${ }^{106}$ Z. Xu, ${ }^{60 b}$ Z. Xu,${ }^{152}$ B. Yabsley, ${ }^{156}$ S. Yacoob, ${ }^{33 a}$ K. Yajima, ${ }^{132}$ D. P. Yallup ${ }^{95}$ N. Yamaguchi, ${ }^{88}$ Y. Yamaguchi, ${ }^{164}$ A. Yamamoto, ${ }^{82}$ M. Yamatani, ${ }^{162}$ T. Yamazaki, ${ }^{162}$ Y. Yamazaki, ${ }^{83}$ J. Yan, ${ }^{60 \mathrm{c}}$ Z. Yan, ${ }^{25}$ H. J. Yang, ${ }^{60 c, 60 \mathrm{~d}}$ H. T. Yang, ${ }^{18}$ S. Yang ${ }^{60 \mathrm{a}}$ T. Yang, ${ }^{63 \mathrm{c}}$ X. Yang, ${ }^{60 b, 58}$ Y. Yang, ${ }^{162} \mathrm{Z}$. Yang, ${ }^{60 \mathrm{a}} \mathrm{W}-\mathrm{M}$. Yao, ${ }^{18}$ Y. C. Yap, ${ }^{46}$ Y. Yasu, ${ }^{82}$ E. Yatsenko, ${ }^{60 c, 60 d}$ H. Ye, ${ }^{15 c}$ J. Ye, ${ }^{42}$ S. Ye,${ }^{29}$ I. Yeletskikh, ${ }^{80}$ M. R. Yexley,${ }^{90}$ E. Yigitbasi, ${ }^{25}$ P. Yin,${ }^{39}$ K. Yorita, ${ }^{178}$ K. Yoshihara, ${ }^{79}$ C. J. S. Young,${ }^{36}$ C. Young, ${ }^{152}$ J. Yu,${ }^{79}$ R. Yuan,${ }^{60 b, o 0}$ X. Yue, ${ }^{61 a}$ M. Zaazoua ${ }^{35 e}$ B. Zabinski, ${ }^{85}$ G. Zacharis, ${ }^{10}$ E. Zaffaroni, ${ }^{54}$ J. Zahreddine, ${ }^{135}$ A. M. Zaitsev, ${ }^{123, i}$ T. Zakareishvili, ${ }^{158 b}$ N. Zakharchuk, ${ }^{34}$ S. Zambito, ${ }^{59}$ D. Zanzi, ${ }^{36}$ D. R. Zaripovas, ${ }^{57}$ S. V. Zeißner, ${ }^{47}$ C. Zeitnitz, ${ }^{181}$ G. Zemaityte, ${ }^{134}$ J. C. Zeng, ${ }^{172}$ O. Zenin, ${ }^{123}$ T. Ženišs, ${ }^{28 a}$ D. Zerwas, ${ }^{65}$ M. Zgubič ${ }^{134}$ B. Zhang, ${ }^{15 \mathrm{c}}$ D. F. Zhang, ${ }^{15 \mathrm{~b}}$ G. Zhang, ${ }^{15 \mathrm{~b}}$ J. Zhang, ${ }^{6}$ Kaili. Zhang, ${ }^{15 \mathrm{a}}$ L. Zhang, ${ }^{15 \mathrm{c}}$ L. Zhang, ${ }^{60 \mathrm{a}}$ M. Zhang, ${ }^{172}$ R. Zhang, ${ }^{180}$ S. Zhang, ${ }^{106}$ X. Zhang, ${ }^{60 \mathrm{c}}$ X. Zhang, ${ }^{60 \mathrm{~b}}$ Y. Zhang, ${ }^{15 a, 15 \mathrm{~d}}$ Z. Zhang, ${ }^{63 \mathrm{a}}$ Z. Zhang, ${ }^{65}$ P. Zhao, ${ }^{49}$ Z. Zhao, ${ }^{60 a}$ A. Zhemchugov, ${ }^{80}$ Z. Zheng, ${ }^{106}$ D. Zhong, ${ }^{172}$ B. Zhou, ${ }^{106}$ C. Zhou, ${ }^{180}$ H. Zhou, ${ }^{7}$ M. S. Zhou, ${ }^{15 a, 15 d}$ M. Zhou, ${ }^{154}$ N. Zhou, ${ }^{60 \mathrm{c}}$ Y. Zhou, ${ }^{7}$ C. G. Zhu, ${ }^{60 \mathrm{~b}}$ C. Zhu, ${ }^{15 a, 15 \mathrm{~d}}$ H. L. Zhu, ${ }^{60 \mathrm{a}}$ H. Zhu, ${ }^{15 \mathrm{a}}$ J. Zhu, ${ }^{106}$ Y. Zhu, ${ }^{60 \mathrm{a}}$ X. Zhuang, ${ }^{15 \mathrm{a}}$ K. Zhukov, ${ }^{111}$ V. Zhulanov, ${ }^{122 b, 122 a}$ D. Zieminska, ${ }^{66}$ N. I. Zimine, ${ }^{80}$ S. Zimmermann, ${ }^{52}$ Z. Zinonos, ${ }^{115}$ M. Ziolkowski, ${ }^{150}$ L. Živković, ${ }^{16}$ G. Zobernig, ${ }^{180}$ A. Zoccoli, ${ }^{23 b, 23 a}$ K. Zoch, ${ }^{53}$ T. G. Zorbas, ${ }^{148}$ R. Zou, ${ }^{37}$ and L. Zwalinski ${ }^{36}$

(ATLAS Collaboration) 


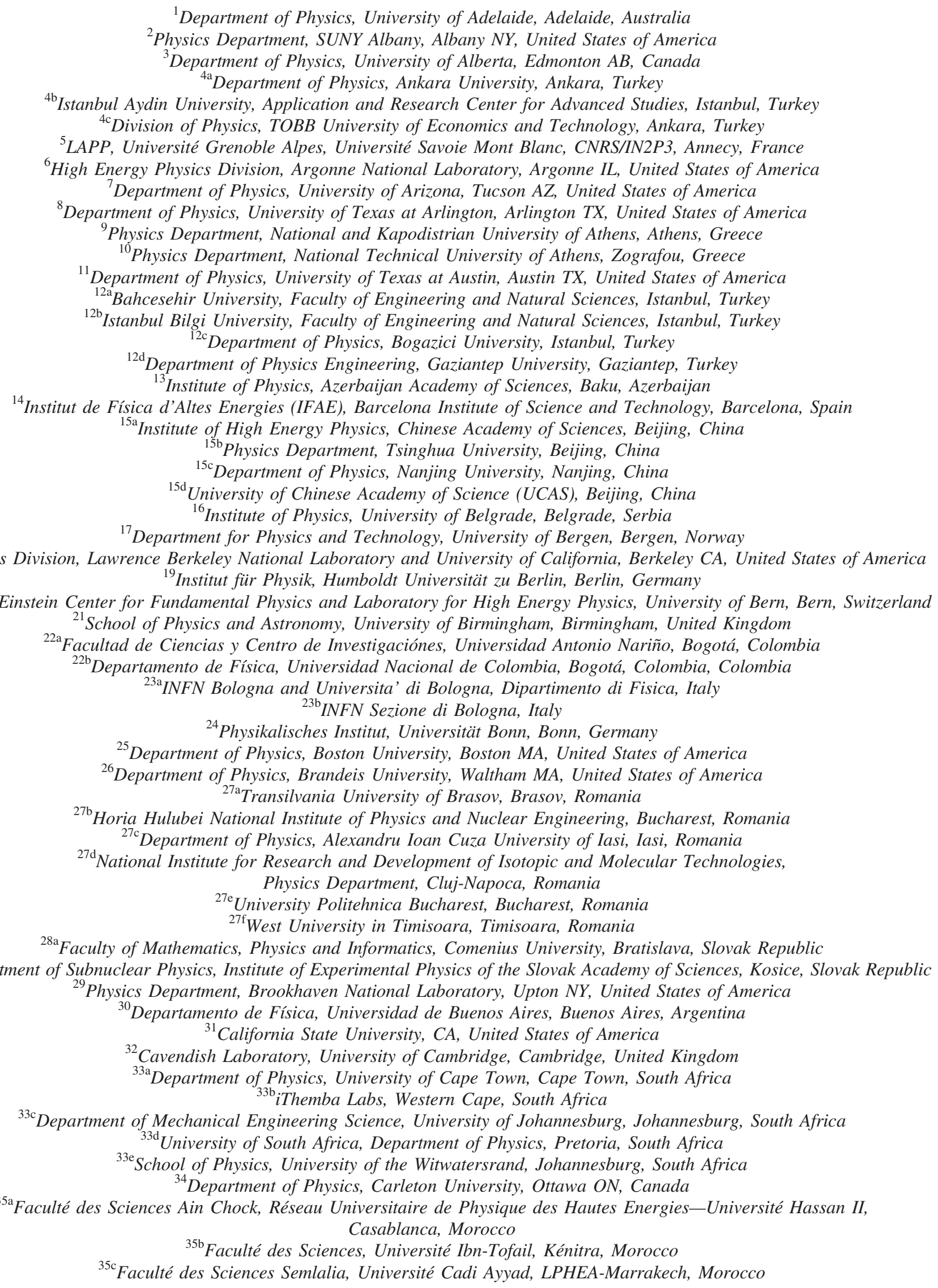




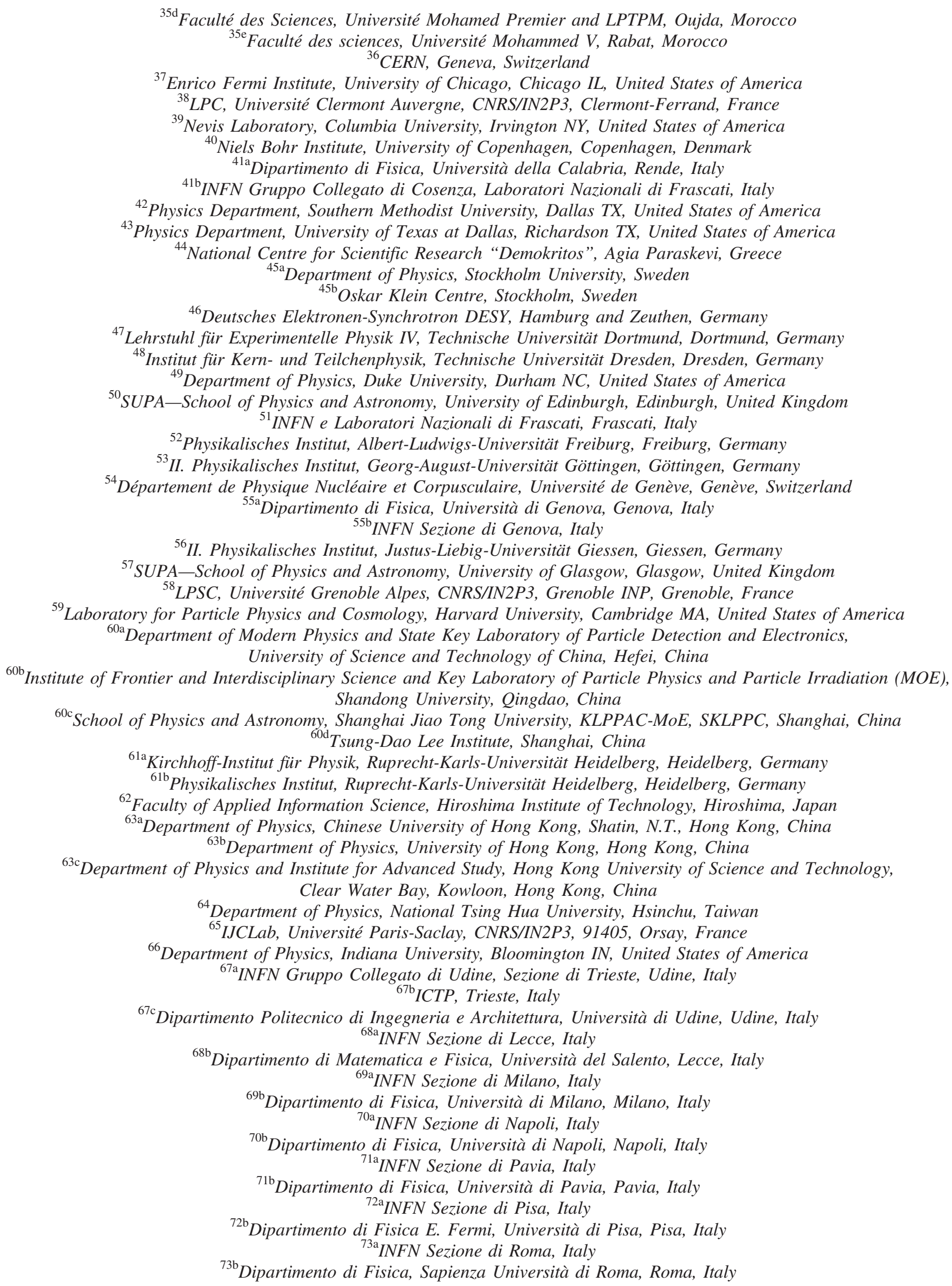




\footnotetext{
${ }^{74 a}$ INFN Sezione di Roma Tor Vergata, Italy

${ }^{74 b}$ Dipartimento di Fisica, Università di Roma Tor Vergata, Roma, Italy

${ }^{75 a}$ INFN Sezione di Roma Tre, Italy

${ }^{75 b}$ Dipartimento di Matematica e Fisica, Università Roma Tre, Roma, Italy

${ }^{76 a}$ INFN-TIFPA, Italy

${ }^{76 \mathrm{~b}}$ Università degli Studi di Trento, Trento, Italy

${ }^{77}$ Institut für Astro- und Teilchenphysik, Leopold-Franzens-Universität, Innsbruck, Austria

${ }^{78}$ University of Iowa, Iowa City IA, United States of America

${ }^{79}$ Department of Physics and Astronomy, Iowa State University, Ames IA, United States of America

${ }^{80}$ Joint Institute for Nuclear Research, Dubna, Russia

${ }^{81 a}$ Departamento de Engenharia Elétrica, Universidade Federal de Juiz de Fora (UFJF), Juiz de Fora, Brazil

${ }^{81 \mathrm{~b}}$ Universidade Federal do Rio De Janeiro COPPE/EE/IF, Rio de Janeiro, Brazil

${ }^{81 c}$ Universidade Federal de São João del Rei (UFSJ), São João del Rei, Brazil

${ }^{81 \mathrm{~d}}$ Instituto de Física, Universidade de São Paulo, São Paulo, Brazil

${ }^{82}$ KEK, High Energy Accelerator Research Organization, Tsukuba, Japan

${ }^{83}$ Graduate School of Science, Kobe University, Kobe, Japan

${ }^{84 \mathrm{a}}$ AGH University of Science and Technology, Faculty of Physics and Applied Computer Science, Krakow, Poland

${ }^{84 \mathrm{~b}}$ Marian Smoluchowski Institute of Physics, Jagiellonian University, Krakow, Poland

${ }^{85}$ Institute of Nuclear Physics Polish Academy of Sciences, Krakow, Poland

${ }^{86}$ Faculty of Science, Kyoto University, Kyoto, Japan

${ }^{87}$ Kyoto University of Education, Kyoto, Japan

${ }^{88}$ Research Center for Advanced Particle Physics and Department of Physics, Kyushu University, Fukuoka, Japan

${ }^{89}$ Instituto de Física La Plata, Universidad Nacional de La Plata and CONICET, La Plata, Argentina

${ }^{90}$ Physics Department, Lancaster University, Lancaster, United Kingdom

${ }^{91}$ Oliver Lodge Laboratory, University of Liverpool, Liverpool, United Kingdom

${ }^{92}$ Department of Experimental Particle Physics, Jožef Stefan Institute and Department of Physics, University of Ljubljana, Ljubljana, Slovenia

${ }^{93}$ School of Physics and Astronomy, Queen Mary University of London, London, United Kingdom

${ }^{94}$ Department of Physics, Royal Holloway University of London, Egham, United Kingdom

${ }^{95}$ Department of Physics and Astronomy, University College London, London, United Kingdom

${ }^{96}$ Louisiana Tech University, Ruston LA, United States of America

${ }^{97}$ Fysiska institutionen, Lunds universitet, Lund, Sweden

${ }^{98}$ Centre de Calcul de l'Institut National de Physique Nucléaire et de Physique des Particules (IN2P3), Villeurbanne, France

${ }^{99}$ Departamento de Física Teorica C-15 and CIAFF, Universidad Autónoma de Madrid, Madrid, Spain

${ }^{100}$ Institut für Physik, Universität Mainz, Mainz, Germany

${ }^{101}$ School of Physics and Astronomy, University of Manchester, Manchester, United Kingdom

${ }^{102}$ CPPM, Aix-Marseille Université, CNRS/IN2P3, Marseille, France

${ }^{103}$ Department of Physics, University of Massachusetts, Amherst MA, United States of America

${ }^{104}$ Department of Physics, McGill University, Montreal QC, Canada

${ }^{105}$ School of Physics, University of Melbourne, Victoria, Australia

${ }^{106}$ Department of Physics, University of Michigan, Ann Arbor MI, United States of America

${ }^{107}$ Department of Physics and Astronomy, Michigan State University, East Lansing MI, United States of America

${ }^{108}$ B.I. Stepanov Institute of Physics, National Academy of Sciences of Belarus, Minsk, Belarus

${ }^{109}$ Research Institute for Nuclear Problems of Byelorussian State University, Minsk, Belarus

${ }^{110}$ Group of Particle Physics, University of Montreal, Montreal QC, Canada

${ }^{111}$ P.N. Lebedev Physical Institute of the Russian Academy of Sciences, Moscow, Russia

${ }^{112}$ National Research Nuclear University MEPhI, Moscow, Russia

${ }^{113}$ D.V. Skobeltsyn Institute of Nuclear Physics, M.V. Lomonosov Moscow State University, Moscow, Russia

${ }^{114}$ Fakultät für Physik, Ludwig-Maximilians-Universität München, München, Germany

${ }^{115}$ Max-Planck-Institut für Physik (Werner-Heisenberg-Institut), München, Germany

${ }^{116}$ Nagasaki Institute of Applied Science, Nagasaki, Japan

${ }^{117}$ Graduate School of Science and Kobayashi-Maskawa Institute, Nagoya University, Nagoya, Japan

${ }^{118}$ Department of Physics and Astronomy, University of New Mexico, Albuquerque NM, United States of America

${ }^{119}$ Institute for Mathematics, Astrophysics and Particle Physics, Radboud University Nijmegen/Nikhef, Nijmegen, Netherlands

${ }^{120}$ Nikhef National Institute for Subatomic Physics and University of Amsterdam, Amsterdam, Netherlands

${ }^{121}$ Department of Physics, Northern Illinois University, DeKalb IL, United States of America

${ }^{122 a}$ Budker Institute of Nuclear Physics and NSU, SB RAS, Novosibirsk, Russia

${ }^{122 \mathrm{~b}}$ Novosibirsk State University Novosibirsk, Russia

${ }^{123}$ Institute for High Energy Physics of the National Research Centre Kurchatov Institute, Protvino, Russia
} 
${ }^{124}$ Institute for Theoretical and Experimental Physics named by A.I. Alikhanov of National Research Centre "Kurchatov Institute”, Moscow, Russia

${ }^{125}$ Department of Physics, New York University, New York NY, United States of America

${ }^{126}$ Ochanomizu University, Otsuka, Bunkyo-ku, Tokyo, Japan

${ }^{127}$ Ohio State University, Columbus OH, United States of America

${ }^{128}$ Homer L. Dodge Department of Physics and Astronomy, University of Oklahoma, Norman OK, United States of America

${ }^{129}$ Department of Physics, Oklahoma State University, Stillwater OK, United States of America

${ }^{130}$ Palacký University, RCPTM, Joint Laboratory of Optics, Olomouc, Czech Republic

${ }^{131}$ Institute for Fundamental Science, University of Oregon, Eugene, OR, United States of America

${ }^{132}$ Graduate School of Science, Osaka University, Osaka, Japan

${ }^{133}$ Department of Physics, University of Oslo, Oslo, Norway

${ }^{134}$ Department of Physics, Oxford University, Oxford, United Kingdom

${ }^{135}$ LPNHE, Sorbonne Université, Université de Paris, CNRS/IN2P3, Paris, France

${ }^{136}$ Department of Physics, University of Pennsylvania, Philadelphia PA, United States of America

${ }^{137}$ Konstantinov Nuclear Physics Institute of National Research Centre "Kurchatov Institute", PNPI, St. Petersburg, Russia

${ }^{138}$ Department of Physics and Astronomy, University of Pittsburgh, Pittsburgh PA, United States of America

${ }^{139 a}$ Laboratório de Instrumentação e Física Experimental de Partículas-LIP, Lisboa, Portugal

${ }^{139 b}$ Departamento de Física, Faculdade de Ciências, Universidade de Lisboa, Lisboa, Portugal

${ }^{139 \mathrm{c}}$ Departamento de Física, Universidade de Coimbra, Coimbra, Portugal

${ }^{139 d}$ Centro de Física Nuclear da Universidade de Lisboa, Lisboa, Portugal

${ }^{139 \mathrm{e}}$ Departamento de Física, Universidade do Minho, Braga, Portugal

${ }^{139 \mathrm{f}}$ Departamento de Física Teórica y del Cosmos, Universidad de Granada, Granada, Spain

${ }^{139 g}$ Dep Física and CEFITEC of Faculdade de Ciências e Tecnologia, Universidade Nova de Lisboa, Caparica, Portugal

${ }^{139 \mathrm{~h}}$ Instituto Superior Técnico, Universidade de Lisboa, Lisboa, Portugal

${ }^{140}$ Institute of Physics of the Czech Academy of Sciences, Prague, Czech Republic

${ }^{141}$ Czech Technical University in Prague, Prague, Czech Republic

${ }^{142}$ Charles University, Faculty of Mathematics and Physics, Prague, Czech Republic

${ }^{143}$ Particle Physics Department, Rutherford Appleton Laboratory, Didcot, United Kingdom

${ }^{144}$ IRFU, CEA, Université Paris-Saclay, Gif-sur-Yvette, France

${ }^{145}$ Santa Cruz Institute for Particle Physics, University of California Santa Cruz, Santa Cruz CA, United States of America

${ }^{146 a}$ Departamento de Física, Pontificia Universidad Católica de Chile, Santiago, Chile

${ }^{146 \mathrm{~b}}$ Universidad Andres Bello, Department of Physics, Santiago, Chile

${ }^{146 c}$ Instituto de Alta Investigación, Universidad de Tarapacá, Chile

${ }^{146 \mathrm{~d}}$ Departamento de Física, Universidad Técnica Federico Santa María, Valparaíso, Chile

${ }^{147}$ Department of Physics, University of Washington, Seattle WA, United States of America

${ }^{148}$ Department of Physics and Astronomy, University of Sheffield, Sheffield, United Kingdom

${ }^{149}$ Department of Physics, Shinshu University, Nagano, Japan

${ }^{150}$ Department Physik, Universität Siegen, Siegen, Germany

${ }^{151}$ Department of Physics, Simon Fraser University, Burnaby BC, Canada

${ }^{152}$ SLAC National Accelerator Laboratory, Stanford CA, United States of America

${ }^{153}$ Physics Department, Royal Institute of Technology, Stockholm, Sweden

${ }^{154}$ Departments of Physics and Astronomy, Stony Brook University, Stony Brook NY, United States of America

${ }^{155}$ Department of Physics and Astronomy, University of Sussex, Brighton, United Kingdom

${ }^{156}$ School of Physics, University of Sydney, Sydney, Australia

${ }^{157}$ Institute of Physics, Academia Sinica, Taipei, Taiwan

${ }^{158 a}$ E. Andronikashvili Institute of Physics, Iv. Javakhishvili Tbilisi State University, Tbilisi, Georgia

${ }^{158 \mathrm{~b}}$ High Energy Physics Institute, Tbilisi State University, Tbilisi, Georgia

${ }^{159}$ Department of Physics, Technion, Israel Institute of Technology, Haifa, Israel

${ }^{160}$ Raymond and Beverly Sackler School of Physics and Astronomy, Tel Aviv University, Tel Aviv, Israel

${ }^{161}$ Department of Physics, Aristotle University of Thessaloniki, Thessaloniki, Greece

${ }^{162}$ International Center for Elementary Particle Physics and Department of Physics, University of Tokyo, Tokyo, Japan

${ }^{163}$ Graduate School of Science and Technology, Tokyo Metropolitan University, Tokyo, Japan

${ }^{164}$ Department of Physics, Tokyo Institute of Technology, Tokyo, Japan

${ }^{165}$ Tomsk State University, Tomsk, Russia

${ }^{166}$ Department of Physics, University of Toronto, Toronto ON, Canada

${ }^{167 \mathrm{a}}$ TRIUMF, Vancouver BC, Canada

${ }^{167 \mathrm{~b}}$ Department of Physics and Astronomy, York University, Toronto ON, Canada

${ }^{168}$ Division of Physics and Tomonaga Center for the History of the Universe, Faculty of Pure and Applied Sciences, University of Tsukuba, Tsukuba, Japan

${ }^{169}$ Department of Physics and Astronomy, Tufts University, Medford MA, United States of America 
${ }^{170}$ Department of Physics and Astronomy, University of California Irvine, Irvine CA, United States of America

${ }^{171}$ Department of Physics and Astronomy, University of Uppsala, Uppsala, Sweden

${ }^{172}$ Department of Physics, University of Illinois, Urbana IL, United States of America

${ }^{173}$ Instituto de Física Corpuscular (IFIC), Centro Mixto Universidad de Valencia-CSIC, Valencia, Spain

${ }^{174}$ Department of Physics, University of British Columbia, Vancouver BC, Canada

${ }^{175}$ Department of Physics and Astronomy, University of Victoria, Victoria BC, Canada

${ }^{176}$ Fakultät für Physik und Astronomie, Julius-Maximilians-Universität Würzburg, Würzburg, Germany

${ }^{177}$ Department of Physics, University of Warwick, Coventry, United Kingdom

${ }^{178}$ Waseda University, Tokyo, Japan

${ }^{179}$ Department of Particle Physics, Weizmann Institute of Science, Rehovot, Israel

${ }^{180}$ Department of Physics, University of Wisconsin, Madison WI, United States of America

${ }^{181}$ Fakultät für Mathematik und Naturwissenschaften, Fachgruppe Physik, Bergische Universität Wuppertal, Wuppertal, Germany

${ }^{182}$ Department of Physics, Yale University, New Haven CT, United States of America

${ }^{\mathrm{a}}$ Deceased.

${ }^{\mathrm{b}}$ Also at Department of Physics, King's College London, London, United Kingdom.

${ }^{\mathrm{c}}$ Also at Instituto de Fisica Teorica, IFT-UAM/CSIC, Madrid, Spain.

dAlso at TRIUMF, Vancouver BC, Canada.

${ }^{\mathrm{e}}$ Also at Department of Physics and Astronomy, University of Louisville, Louisville, KY, United States of America.

${ }_{\mathrm{f}}^{\mathrm{f}}$ Also at Physics Department, An-Najah National University, Nablus, Palestine.

${ }^{\mathrm{g}}$ Also at Department of Physics, University of Fribourg, Fribourg, Switzerland.

${ }^{h}$ Also at Departament de Fisica de la Universitat Autonoma de Barcelona, Barcelona, Spain.

${ }^{\mathrm{i}}$ Also at Moscow Institute of Physics and Technology State University, Dolgoprudny, Russia.

${ }^{\mathrm{j}}$ Also at Department of Physics, Ben Gurion University of the Negev, Beer Sheva, Israel.

${ }^{\mathrm{k}}$ Also at Universita di Napoli Parthenope, Napoli, Italy.

${ }^{1}$ Also at Institute of Particle Physics (IPP), Vancouver, Canada.

${ }^{\mathrm{m}}$ Also at Department of Physics, University of Adelaide, Adelaide, Australia.

${ }^{\mathrm{n}}$ Also at Dipartimento di Matematica, Informatica e Fisica, Università di Udine, Udine, Italy.

${ }^{\circ}$ Also at Department of Physics, St. Petersburg State Polytechnical University, St. Petersburg, Russia.

${ }^{\mathrm{p}}$ Also at Borough of Manhattan Community College, City University of New York, New York NY, United States of America.

${ }^{\mathrm{q}}$ Also at Department of Physics, California State University, Fresno, United States of America.

${ }^{\mathrm{r}}$ Also at Department of Financial and Management Engineering, University of the Aegean, Chios, Greece.

${ }^{\mathrm{s}}$ Also at Department of Physics, California State University, East Bay, United States of America.

${ }^{t}$ Also at Institucio Catalana de Recerca i Estudis Avancats, ICREA, Barcelona, Spain.

uAlso at IJCLab, Université Paris-Saclay, CNRS/IN2P3, 91405, Orsay, France.

${ }^{v}$ Also at Graduate School of Science, Osaka University, Osaka, Japan.

${ }^{w}$ Also at Physikalisches Institut, Albert-Ludwigs-Universität Freiburg, Freiburg, Germany.

${ }^{\mathrm{x}}$ Also at Institute of Physics, Azerbaijan Academy of Sciences, Baku, Azerbaijan.

${ }^{y}$ Also at Institute for Mathematics, Astrophysics and Particle Physics, Radboud University Nijmegen/Nikhef, Nijmegen, Netherlands.

${ }^{\mathrm{z}}$ Also at CERN, Geneva, Switzerland.

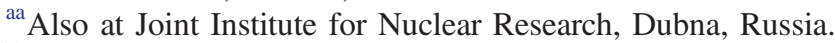

${ }^{\mathrm{bb}}$ Also at Hellenic Open University, Patras, Greece.

${ }^{c c}$ Also at The City College of New York, New York NY, United States of America.

${ }^{\mathrm{dd}}$ Also at Department of Physics, California State University, Sacramento, United States of America.

${ }^{e e}$ Also at Département de Physique Nucléaire et Corpusculaire, Université de Genève, Genève, Switzerland.

${ }^{\mathrm{ff}}$ Also at Louisiana Tech University, Ruston LA, United States of America.

${ }^{g g}$ Also at Institute for Nuclear Research and Nuclear Energy (INRNE) of the Bulgarian Academy of Sciences, Sofia, Bulgaria.

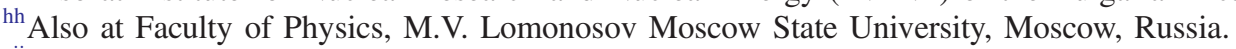

${ }^{\text {ii }}$ Also at Department of Applied Physics and Astronomy, University of Sharjah, Sharjah, United Arab Emirates.

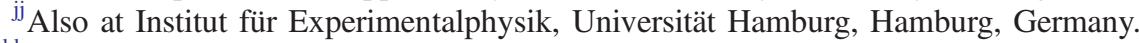

${ }^{\mathrm{kk}}$ Also at CPPM, Aix-Marseille Université, CNRS/IN2P3, Marseille, France.

${ }^{11}$ Also at National Research Nuclear University MEPhI, Moscow, Russia.

${ }^{\mathrm{mm}}$ Also at Institute for Particle and Nuclear Physics, Wigner Research Centre for Physics, Budapest, Hungary.

${ }^{\mathrm{nn}}$ Also at Giresun University, Faculty of Engineering, Giresun, Turkey.

${ }^{\circ o}$ Also at Department of Physics and Astronomy, Michigan State University, East Lansing MI, United States of America. 